\title{
The ALMA-PILS survey: inventory of complex organic molecules towards IRAS 16293-2422 A
}

\author{
S. Manigand ${ }^{1}$, J. K. Jørgensen ${ }^{1}$, H. Calcutt ${ }^{2}$, H. S. P. Müller ${ }^{3}$, N. F. W. Ligterink ${ }^{4}$, A. Coutens ${ }^{5}$, \\ M. N. Drozdovskaya ${ }^{4}$, E. F. van Dishoeck ${ }^{6,7}$, and S. F. Wampfler ${ }^{4}$ \\ ${ }^{1}$ Niels Bohr Institute \& Centre for Star and Planet Formation, University of Copenhagen, Øster Voldgade 5-7, \\ 1350 Copenhagen K., Denmark \\ e-mail: sebastien@nbi.ku.dk \\ 2 Department of Space, Earth and Environment, Chalmers University of Technology, 41296 Gothenburg, Sweden \\ ${ }^{3}$ I. Physikalisches Institut, Universität zu Köln, Zülpicher Str. 77, 50937 Köln, Germany \\ ${ }^{4}$ Center for Space and Habitability (CSH), University of Bern, Gesellschaftsstrasse 6, 3012 Bern, Switzerland \\ ${ }^{5}$ Laboratoire d'Astrophysique de Bordeaux, Univ. Bordeaux, CNRS, B18N, allée Geoffroy Saint-Hilaire, 33615 Pessac, France \\ ${ }^{6}$ Leiden Observatory, Leiden University, PO Box 9513, 2300 RA Leiden, The Netherlands \\ ${ }^{7}$ Max-Planck Institut für Extraterrestrische Physik (MPE), Giessenbachstr. 1, 85748 Garching, Germany
}

Received 12 July 2019 / Accepted 2 January 2020

\begin{abstract}
Context. Complex organic molecules are detected in many sources in the warm inner regions of envelopes surrounding deeply embedded protostars. Exactly how these species form remains an open question.

Aims. This study aims to constrain the formation of complex organic molecules through comparisons of their abundances towards the Class 0 protostellar binary IRAS 16293-2422.

Methods. We utilised observations from the ALMA Protostellar Interferometric Line Survey of IRAS 16293-2422. The species identification and the rotational temperature and column density estimation were derived by fitting the extracted spectra towards IRAS 16293-2422 A and IRAS 16293-2422 B with synthetic spectra. The majority of the work in this paper pertains to the analysis of IRAS 16293-2422 A for a comparison with the results from the other binary component, which have already been published.

Results. We detect 15 different complex species, as well as 16 isotopologues towards the most luminous companion protostar IRAS 16293-2422 A. Tentative detections of an additional 11 isotopologues are reported. We also searched for and report on the first detections of methoxymethanol $\left(\mathrm{CH}_{3} \mathrm{OCH}_{2} \mathrm{OH}\right)$ and trans-ethyl methyl ether $\left(\mathrm{t}-\mathrm{C}_{2} \mathrm{H}_{5} \mathrm{OCH}_{3}\right)$ towards IRAS $16293-2422 \mathrm{~B}$ and the follow-up detection of deuterated isotopologues of acetaldehyde $\left(\mathrm{CH}_{2} \mathrm{DCHO}\right.$ and $\left.\mathrm{CH}_{3} \mathrm{CDO}\right)$. Twenty-four lines of doubly-deuterated methanol $\left(\mathrm{CHD}_{2} \mathrm{OH}\right)$ are also identified.

Conclusions. The comparison between the two protostars of the binary system shows significant differences in abundance for some of the species, which are partially correlated to their spatial distribution. The spatial distribution is consistent with the sublimation temperature of the species; those with higher expected sublimation temperatures are located in the most compact region of the hot corino towards IRAS 16293-2422 A. This spatial differentiation is not resolved in IRAS 16293-2422 B and will require observations at a higher angular resolution. In parallel, the list of identified $\mathrm{CHD}_{2} \mathrm{OH}$ lines shows the need of accurate spectroscopic data including their line strength.
\end{abstract}

Key words. astrochemistry - stars: formation - stars: protostars - ISM: molecules - ISM: individual objects: IRAS 16293-2422

\section{Introduction}

Complex organic molecules (COMs, i.e. molecules containing six or more atoms with at least one carbon atom; Herbst \& van Dishoeck 2009) are observed in various interstellar environments, ranging from very cold, dense collapsing cloud cores (e.g. Bacmann et al. 2012; Taquet et al. 2017) to protoplanetary discs (e.g. Öberg et al. 2015; Walsh et al. 2016; Favre et al. 2018), as well as in low- and high-mass star-forming regions (e.g. Blake et al. 1987; Fayolle et al. 2015; Bergner et al. 2017; Ceccarelli et al. 2017; Ospina-Zamudio et al. 2018; Bøgelund et al. 2019). Meteorite measurements (Ehrenfreund et al. 2001; Botta \& Bada 2002), comet coma observations (Bockelée-Morvan et al. 2000; Crovisier et al. 2004; Biver et al. 2014), and in situ Rosetta mission measurements (Le Roy et al. 2015; Altwegg et al. 2017) have revealed complex organic molecules, such as glycolaldehyde $\left(\mathrm{CH}_{2} \mathrm{OHCHO}\right)$, ethylene glycol $\left(\left(\mathrm{CH}_{2} \mathrm{OH}\right)_{2}\right)$, and glycine. The presence of complex and even pre-biotic species suggests that some of these molecules that formed during the earliest stage of stellar formation are preserved until the formation of small bodies. The extent to which the most complex species were formed at the earliest evolutionary stage of the protosolar nebula and how they were preserved or transformed during the formation of planetesimals remain open and complex questions.

In this study, we focus on the formation of COMs during the deeply embedded Class 0 stage. Class 0 protostars are characterised by an envelope that is rich in diverse molecules, where most of the mass of the system is gathered (Andre \& Montmerle 1994). COMs may be observed in the inner regions of the envelope, which is close to their host protostar, where the temperature exceeds the water ice desorption temperature of $\sim 100 \mathrm{~K}$. These molecule-rich regions are called hot cores for high-mass protostars and hot corinos for low-mass counterparts. At sub-millimetre wavelengths, most of the lines originate 
from complex species. The measured COM abundances are typically between $10^{-7}$ and $10^{-11}$ with respect to $\mathrm{H}_{2}$ (e.g. Herbst \& van Dishoeck 2009). In general, the formation of COMs is thought to occur on ice surfaces through a succession of different processes that are initiated by efficient hydrogenation of simple ice species, for example, $\mathrm{CO}$, which leads to the efficient production of methanol $\left(\mathrm{CH}_{3} \mathrm{OH}\right.$; Garrod \& Herbst 2006; Garrod et al. 2008; Fuchs et al. 2009; Chuang et al. 2018). During the collapse of the pre-stellar core, the temperature starts to increase during the so-called warm-up phase, and more complex species form and desorb into the gas phase in the hot corino. The resulting abundances of these species in the gas depend on the initial abundances of the precursors in the parent cloud, but they are also very sensitive to the physical conditions of the prestellar and protostellar phases and may thus vary significantly for different protostellar sources (Garrod 2013; Drozdovskaya et al. 2016). While the gas-phase formation pathways were not efficient enough to explain such high abundances of the COMs observed in the dense warm envelope, the recent progress in quantum chemical modelling brought attention back to the gasphase chemistry (e.g. Balucani et al. 2015; Skouteris et al. 2018, 2019). Similar progress has been made recently in the study of the deuteration of COMs through gas-phase reactions (Skouteris et al. 2017). Careful comparisons between observations and predictions from grain surface and gas-phase reaction experiments and calculations are needed to address the relative importance for the species that are present in their different protostellar environments.

Close protostellar multiple sources, that is, systems with multiple components separated by $\lesssim 1000$ au, are particularly interesting laboratories for studying the effect of the physical conditions with time. Indeed, the chemical evolution of such sources is expected to stem from the same initial compositions and physical conditions (gas and dust temperatures as well as ultra-violet irradiation), which are inherited from the common parental cloud. The Class 0 low-mass protostellar binary IRAS 16293-2422 (IRAS 16293 hereafter), which is located in the $\rho$ Ophiuchus cloud complex at a distance of $\sim 140 \mathrm{pc}$ (Dzib et al. 2018), is one of the best sources to investigate for that purpose. The components of the protostellar binary are known to be rich in COMs with abundances that are comparable to those seen towards high-mass protostars (van Dishoeck et al. 1995; Cazaux et al. 2003; Caux et al. 2011). Previous interferometric observations revealed abundance differences of specific molecules between the two protostars (Bisschop et al. 2008; Jørgensen et al. 2011, 2012); however, the origin of the differentiation is still debated. The Atacama Large Millimetre/ submillimetre Array (ALMA) Protostellar Interferometric Line Survey $^{1}$ (PILS, Jørgensen et al. 2016) performed an unbiased spectral survey of the system in Band 7, demonstrating the strength of ALMA for detecting new complex species towards the most compact component IRAS $16293 \mathrm{~B}$ of the system, including $\mathrm{CH}_{3} \mathrm{Cl}$ (Fayolle et al. 2017), $\mathrm{CH}_{3} \mathrm{NC}$ (Calcutt et al. 2018a), HONO (Coutens et al. 2019), $\mathrm{NH}_{2} \mathrm{CN}$ (Coutens et al. 2018), and $\mathrm{CH}_{3} \mathrm{NCO}$ (Ligterink et al. 2017). In addition, many deuterated and less abundant isotopologues have been found for the first time in the interstellar medium (ISM) towards this source, including $\mathrm{NHDCHO}, \mathrm{NH}_{2} \mathrm{CDO}$ (Coutens et al. 2016), NHDCN (Coutens et al. 2018), $\mathrm{CHD}_{2} \mathrm{CN}$ (Calcutt et al. 2018b), deuterated and ${ }^{13} \mathrm{C}$ isotopologues of $\mathrm{CH}_{2} \mathrm{OHCHO}$, deuterated ethanol $\left(\mathrm{C}_{2} \mathrm{H}_{5} \mathrm{OH}\right)$, ketene $\left(\mathrm{CH}_{2} \mathrm{CO}\right)$, acetaldehyde $\left(\mathrm{CH}_{3} \mathrm{CHO}\right)$

\footnotetext{
1 The reduced dataset and all the publications are available on the website http://youngstars.nbi.dk/PILS/.
}

and formic acid (HCOOH; Jørgensen et al. 2016, 2018), $\mathrm{OC}^{33} \mathrm{~S}$ (Drozdovskaya et al. 2018), and doubly deuterated methyl formate $\left(\mathrm{CHD}_{2} \mathrm{OCHO}\right.$; Manigand et al. 2019). This paper aims to study the O-bearing COMs in the other protostar IRAS 16293 A of the binary system and to compare their abundances with IRAS 16293 B. Because of the proximity of the two sources (5"; $\sim 720 \mathrm{au}$ ), the two sources are expected to have the same molecular heritage as their parent cloud. Therefore, any abundance difference between the sources could reveal the chemistry that occurred during the earliest evolutionary phases of this low-mass protostellar binary.

Similar comparative studies have been carried out towards the Class 0 binary protostar NGC 1333 IRAS 4A. LópezSepulcre et al. (2017) observed this source at high angular resolution with ALMA and measured the abundances of eight COMs towards the two components of the binary. The authors notice a large difference in abundance for all the species and suggest that the lack of COMs towards one of the source could be due to a lower mass, with a lower accretion rate, compared to the molecule rich counterpart. This scenario could alternatively suggest that the COM-poor source is not yet in the collapse phase resulting in the formation of the hot corino, which is consistent with the lack of evidence for the presence of a hot corino in this component (Persson et al. 2012). In contrast, both components in IRAS 16293 are known to harbour hot corinos.

In this study, we present an analysis of the PILS data taken towards IRAS $16293 \mathrm{~A}$, which focus on the content of the Obearing molecules in the hot corino region. This work aims to compare the abundances found for both components of the binary system to better understand the structure and/or the chemistry of this particular binary source. This paper is organised as follows. In Sect. 2, we describe the observations and spectroscopic data used in this study. In Sect. 3, we present the results of the observations and analyse the spectrum. In Sect. 4, we compare the abundances with those in IRAS $16293 \mathrm{~B}$ and discuss the different aspects of the two sources. Finally, we summarise the key points of the analysis and the discussion in Sect. 5.

\section{Data analysis}

In this section, we present the results of the oxygen-bearing COMs identification using the local thermodynamic equilibrium (LTE) analysis. In Sect. 2.1 we present the observations used in this study and the species identification is described in Sect. 2.2. The LTE model and the $\chi^{2}$-fitting are detailed in Sects. 2.3 and 2.4 , respectively. Finally, the treatment of the uncertainties and the continuum correction are addressed in Sect. 2.5.

\subsection{Observations}

We used ALMA data from the PILS survey of the low-mass protostellar binary IRAS 16293 (Jørgensen et al. 2016). The data are a combination of the $12 \mathrm{~m}$ dishes and the Atacama Compact Array (ACA). The observations cover the frequency range of 329.1-362.9 GHz with a spectral resolution of $0.244 \mathrm{MHz}$, corresponding to $\sim 0.2 \mathrm{~km} \mathrm{~s}^{-1}$, and a restoring beam of 0.5 . The survey reaches a sensitivity of 4-5 mJy beam ${ }^{-1} \mathrm{~km} \mathrm{~s}^{-1}$ across the entire frequency range. The ALMA software suite CASA (McMullin et al. 2007) was used to reduce the data, except for the continuum subtraction, which was performed after the data reduction (for more details, see Jørgensen et al. 2016). The flux calibration across the spectral range is consistent with the 5\% level. The spectroscopic data of all the species that are analysed in this paper are detailed in Appendix $\mathrm{C}$ as well as the vibrational 


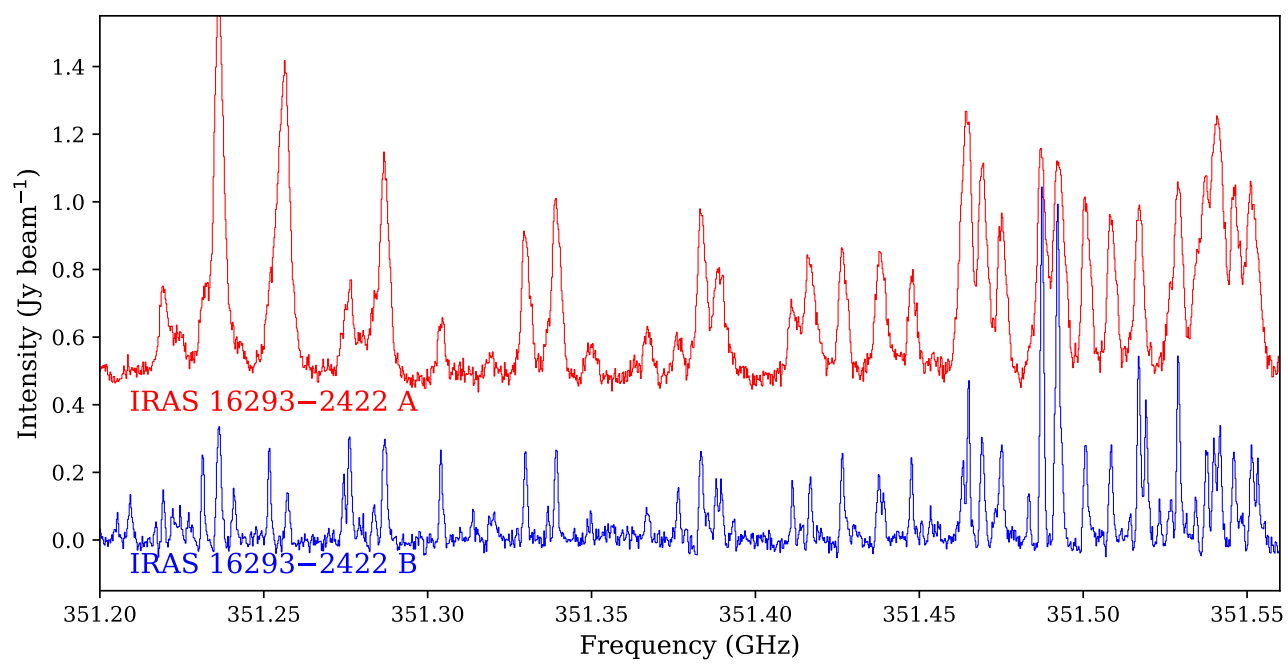

Fig. 1. Portion of the spectrum towards IRAS 16293 A and IRAS 16293 B in red and blue, respectively, extracted on their corresponding offset positions. IRAS $16293 \mathrm{~A}$ spectrum is offset by $0.5 \mathrm{Jy} \mathrm{beam}^{-1}$ for illustrative purposes.

correction factors used, in the case of rotational temperatures that are higher than $100 \mathrm{~K}$.

\subsection{Species identification}

In this study, oxygen-bearing COMs have been investigated towards IRAS 16293 A and IRAS 16293 B through the analysis of the spectral emission at one position towards each source. The continuum peak positions, the strong continuum emission, the dynamics of the gas, and the spectral line density make it difficult to analyse the molecular emission. Therefore, the spectra were extracted towards offset positions at $0 . ' 6$ from the continuum peak position in the north-east direction and 0.'5 in the south-west direction for IRAS 16293 A and B, respectively. This is in agreement with the choice of the extracted spectrum position in the previous PILS studies (e.g. Calcutt et al. 2018b; Jørgensen et al. 2018; Lykke et al. 2017). Figure 1 shows the spectra towards IRAS $16293 \mathrm{~A}$ and B. Most of the lines identified towards IRAS 16293 B also appear in IRAS 16293 A, albeit at different intensities, which indicates that IRAS 16293 A is as rich in molecules as its companion. The line widths are typically two to three times larger towards IRAS 16293 A than towards IRAS $16293 \mathrm{~B}$, thereby increasing the confusion due to blending effects.

The complexity of the molecular emission towards IRAS16293 A and B makes the line assignment to the species difficult when based on the peak frequency alone. In some cases, a species that was not considered to be present in the source might have a significant number of transitions with frequencies coinciding with bright transitions of other species. In order to deal with such line confusion, we coupled the identification of possible O-bearing COMs present in the gas to the LTE analysis. All the species detected in the counterpart protostar IRAS16293 B and their fainter isotopologues were considered as potential candidate species that compose the envelope of IRAS16293 A. Additionally, related and more complex O-bearing COMs have also been added to the list of candidates, such as trans-ethyl methyl ether $\left(\mathrm{t}-\mathrm{C}_{2} \mathrm{H}_{5} \mathrm{OCH}_{3}\right)$ and methoxymethanol $\left(\mathrm{CH}_{3} \mathrm{OCH}_{2} \mathrm{OH}\right)$.

\subsection{LTE model and detection criteria}

We compared the extracted spectrum with a synthetic model in order to identify and derive the abundances of species that are present in the gas. The model applies the radiative transfer equations to a homogeneous column of gas along the line of sight under the LTE assumption. Only the optically thin lines, which have a line opacity lower than 0.1 , are considered in the comparison of the modelled spectrum. For each species, a single excitation temperature was assumed to describe the level populations, which we refer to as rotational temperature.

The brightness temperature $T_{\mathrm{b}}(v)$ depends on the line opacity $\tau(v)$, through the relation:

$T_{\mathrm{b}}(v)=T_{0}\left(\Omega \frac{1}{\mathrm{e}^{T_{0} / T_{\text {rot }}}-1}-\frac{1}{\mathrm{e}^{T_{0} / T_{\mathrm{CMB}}}-1}\right)\left(1-\mathrm{e}^{-\tau(v)}\right)$,

with $T_{0}=\mathrm{h} v / k_{\mathrm{B}}, T_{\text {rot }}$, the rotational temperature of the gas and the cosmic microwave background temperature $T_{\mathrm{CMB}}=2.73 \mathrm{~K}$. The filling factors of the gas emission, $\Omega$, is equal to 0.5 (Jørgensen et al. 2016), corresponding to a source size of 0.'5. The source size is the same as what was used in the previous studies of IRAS 16293 B based on the same dataset.

Under the LTE assumption and assuming a Gaussian profile, the line opacity is expressed as:

$$
\begin{aligned}
& \tau(v)=\tau_{0} \exp \frac{-\left(v-v_{\mathrm{LSR}}\right)^{2}}{2}\left(\frac{2 \sqrt{2 \ln 2}}{\Delta v_{\mathrm{FWHM}}}\right)^{2} \\
& \text { and } \tau_{0}=\frac{2 \sqrt{2 \ln 2}}{\Delta v_{\mathrm{FWHM}} \sqrt{2 \pi}} \frac{N_{\mathrm{tot}} g_{\mathrm{u}} A_{\mathrm{ul}} c^{3}}{8 \pi Q\left(T_{\mathrm{rot}}\right) v_{0}^{3}}\left(\mathrm{e}^{T_{0} / T_{\text {rot }}}-1\right) \mathrm{e}^{-\frac{E_{\mathrm{u}}}{\mathrm{k}_{\mathrm{B}} T_{\mathrm{rot}}}},
\end{aligned}
$$

where $g_{\mathrm{u}}$ is the upper state degeneracy, $E_{\mathrm{u}}$ is the upper state energy, $A_{\mathrm{ul}}$ is the Einstein coefficient of the transition, $N_{\text {tot }}$ is the column density, and $Q\left(T_{\text {rot }}\right)$ is the partition function evaluated at $T_{\text {rot }}$. We note that the brightness temperature and the line opacity are functions of the velocity $v$, representing the velocity shift with respect to the peak velocity, $v_{\mathrm{LSR}}$, which was set at the rest frequency of the modelled line. The parameters $T_{\mathrm{ex}}$, $N_{\text {tot }}, v_{\mathrm{LSR}}$ and $\Delta v_{\mathrm{FWHM}}$, the full line width at half-maximum in velocity units, are free parameters in the model.

The detection criteria for a species in the PILS data is mostly affected by the high density of the line present in the spectrum, which is more problematic towards IRAS16293 A due to the broader lines. A line is considered to be unblended, and thus usable for the fit, in the following cases: if there is no line from another species in the $v_{0} \pm 2 F W H M$ frequency range (i.e. 
Rayleigh criteria); and if there is a line in the $v_{0} \pm 2 F W H M$ frequency range but the peak intensity of this line is lower than $10 \%$ the peak intensity of the line of interest or less than $1 \sigma$.

The frequency range considered in the Rayleigh criteria is extended to $\pm 4 F W H M$ at the proximity of a very optically thick blending line, for example, the low- $E_{\text {up }} \mathrm{CH}_{3} \mathrm{OH}$ lines.

\section{4. $\chi^{2}$-fitting}

Towards the analysed position, 0.' 6 north-east of IRAS 16293 A, a line width FWHM of $2.2-2.4 \mathrm{~km} \mathrm{~s}^{-1}$, and a peak velocity $v_{\mathrm{lsr}}$ of $0.8 \mathrm{~km} \mathrm{~s}^{-1}$ reproduce the emission line shapes of all the species in this study, suggesting that they are indeed located in the same region around the protostar. The line width and the peak velocity towards the offset position from IRAS $16293 \mathrm{~B}$, which are 1.0 and $2.7 \mathrm{~km} \mathrm{~s}^{-1}$, respectively, are consistent with the range of values from the previous PILS studies $\left(0.8-1.0 \mathrm{~km} \mathrm{~s}^{-1}\right.$ and 2.5-2.7 $\mathrm{km} \mathrm{s}^{-1}$, respectively, Coutens et al. 2016; Lykke et al. 2017; Calcutt et al. 2018a; Persson et al. 2018; Ligterink et al. 2017).

The species that are considered have been fitted on a grid in column density and rotational temperature, ranging from $10^{14}$ to $10^{18} \mathrm{~cm}^{-2}$ in logarithmic scale and from 75 to $300 \mathrm{~K}$ by increments of $5 \mathrm{~K}$, respectively. Each of them were iteratively fitted from the most to least abundant and put in a reference spectrum that was taken into account for the fit of the next species.

The comparison between the observed spectrum and the model is quantified by the $\chi^{2}$ minimisation, which is defined as

$\chi^{2}=\sum_{\text {lines }} \int_{\text {line profile }} \omega(v)\left(\frac{I_{\mathrm{b}}(v)-T_{\mathrm{b}}(v)}{\sigma_{\mathrm{b}}(v)}\right)^{2} \mathrm{~d} v$,

where $I_{\mathrm{b}}(v)$ and $\sigma_{\mathrm{b}}(v)$ are the line intensity and the intensity uncertainty of the extracted spectrum, respectively. We note that the intensity uncertainty includes the contribution from the calibration uncertainty $\sigma_{\text {cal }}$, the root mean square (RMS) $\sigma_{\text {RMS }}$, and the following: $\sigma_{\mathrm{b}}(v)=\sqrt{\sigma_{\mathrm{RMS}}^{2}(v)+\sigma_{\text {cal }}^{2}(v)}$.

The weighting factor $\omega(v)$ is equal to three if $T_{\mathrm{b}}(v)>I_{\mathrm{b}}(v)$, and it is one otherwise. In the case of this study, this weighting factor provides a better constraint on possible blending effects, which could bias the minimisation. In other words, the weighting factor increases the $\chi^{2}$ value when the model is above the spectrum. We chose to break the symmetry of the model that way to allow the fitting procedure to converge to lower column density values compared to those derived with a symmetric $\chi^{2}$, which could include possible contributions from species that are close enough for blending. The choice of the weighting factor value of three is arbitrary and depends on the degree of line confusion in the data.

\subsection{Uncertainties and continuum corrections}

The uncertainties are usually given by the covariance matrix which is estimated in the standard $\chi^{2}$ minimisation methods. However, the modified $\chi^{2}$ used in this study no longer allows for the use of the covariance matrix to get the uncertainties. Instead, the uncertainties have been estimated using a simple Monte Carlo simulation, where the spectrum as well as an additional Gaussian noise of $\sigma_{\mathrm{b}}(v)$ amplitude were fitted to the synthetic spectrum several times. This gives a distribution of each parameter; here, the excitation temperature and the column density are called the posterior probability distributions. This versatile method has also been successfully used to estimate the uncertainties on the $3 \mu \mathrm{m} \mathrm{OH}$ band model parameters as well as in reflectance spectroscopy of meteorites and asteroids (Potin et al. 2019). In summary, the posterior probability distribution of the parameters represents the distribution of the possible values that can take each parameter, given the uncertainties of the data. The formalism of this Monte Carlo simulation is detailed in Appendix B.

This statistical estimation of the uncertainties gives much lower relative errors on the excitation temperature and column density compared to the actual data relative uncertainties. This suggests that the proper statistical estimate of the errors is not conservative considering the underlying hypotheses of the LTE model and how they are handled in the minimisation. Indeed, the $\chi^{2}$ minimisation assumes that the model is perfect and determines the best set of parameters to fit the observations. However, the observations include many effects that are neglected by the LTE analysis but which appear in the spectrum. These effects can be, for example, non-uniform source coverage, beam dilution, self-absorption effects, or abundance gradients of the species in the line of sight (with the associated velocity shift). Therefore, we chose to take conservative values of $\sim 20$ and $\sim 30 \%$ for the rotational temperatures and the column densities relative uncertainties, respectively, for IRAS 16293 A. For consistency, the relative uncertainty values used in the study of Jørgensen et al. (2018) are assumed for IRAS $16293 \mathrm{~B}$, that is, $\sim 20$ and $\sim 10 \%$ for the rotational temperatures and the column densities, respectively.

The dust emission is treated as a continuum brightness temperature, which is directly derived from the continuum emission in the same frequency range. The high density of both sources makes it so the dust emission is partially coupled to the gas emission. In order to take into account the continuum contribution to the line emission, the correction factor $A_{\text {corr }}$ was applied to the column density

$A_{\text {corr }}=\frac{\Omega \frac{1}{\mathrm{e}^{T_{0} / T_{\text {rot }}}-1}-\frac{1}{\mathrm{e}^{T_{0} / T_{\mathrm{CMB}}-1}}}{\Omega \frac{1}{\mathrm{e}^{T_{0} / T_{\text {rot }}-1}}-\Omega_{\text {dust }} \frac{1}{\mathrm{e}^{T_{0} / T_{\mathrm{d}}}-1}}$

in which $T_{\mathrm{d}}$ is the dust brightness temperature and $\Omega_{\text {dust }}$ is the dust emission filling factor, which is assumed to be equal to $\Omega$. Under the LTE assumption, the use of a correction factor is equivalent to replacing $T_{\mathrm{CMB}}$ with the dust brightness temperature $T_{\mathrm{d}}$ in Eq. (1). The dust brightness temperatures used in this paper are 30.1 and $21.2 \mathrm{~K}$ towards the offset position from continuum peak positions towards IRAS16293 A and $\mathrm{B}$, respectively. The value of the continuum correction factor ranges from $\sim 1.1$ at $300 \mathrm{~K}$ to $\sim 1.4$ at $90 \mathrm{~K}$ and it does not strongly depend on the frequency across the spectral range of the PILS data.

\section{Results}

In total, 15 different species as well as 16 isotopologues, have been securely detected, while 11 additional species and isotopologues are tentatively identified towards IRAS 16293 A. Table 1 shows the result of the LTE analysis with the number of lines taken into account in the minimisation, the best-fit parameters (i.e. $T_{\text {rot }}, N_{\text {tot }}$, and $\Delta v_{\text {FWHM }}$ ), the abundance with respect to the main isotopologue for the same species, and the corresponding isotopic ratio.

\subsection{Abundances and rotational temperatures}

The relative abundances of the oxygen species are determined from their column density relative to the column density of $\mathrm{CH}_{3} \mathrm{OH}$. We note that $\mathrm{H}_{2}$ was not used as a reference for 
the estimation of the abundances because only the lower limit of $\mathrm{H}_{2}$ column density can be determined from the optically thick dust emission. Calcutt et al. (2018b) and Jørgensen et al. (2016) estimated the lower limit of $\mathrm{H}_{2}$ column density towards IRAS $16293 \mathrm{~A}$ and $\mathrm{B}$, respectively. The $\mathrm{D} / \mathrm{H}$ ratio is based on the column density ratio and is corrected from the statistics of $\mathrm{H}$ atoms in the deuterated chemical group of the molecule. For example, the column density ratio of $\mathrm{CH}_{2} \mathrm{DOH}$ is three times higher than its $\mathrm{D} / \mathrm{H}$ ratio. In addition, the statistical correction includes the symmetries in the sense of the rotational spectroscopy. For example, the asymmetric deuterated dimethyl ether $\left(\mathrm{a}-\mathrm{CH}_{2} \mathrm{DOCH}_{3}\right)$ has four possible sites where the $\mathrm{D}$ atom could be placed, that is, out of the $\mathrm{C}-\mathrm{O}-\mathrm{C}$ plane, regardless of the rotational emission of the molecule. Thus, its column density ratio is four times higher than the D/H ratio. Similarly, the symmetric s- $\mathrm{CH}_{2} \mathrm{DOCH}_{3}$ conformer has two in-plane possible sites for the $\mathrm{D}$ atom, which leads to a factor of two between the column density ratio and the $\mathrm{D} / \mathrm{H}$ ratio. The rotational temperature of the isotopologues was fixed to the value derived for their main isotopologue, except for formaldehyde $\left(\mathrm{H}_{2} \mathrm{CO}\right)$ where only the $\mathrm{H}_{2}^{13} \mathrm{CO}$ rotational temperature could be constrained. For several isotopologues, the fit converged to a different rotational temperature as the main isotopologue. The difference is lower than the uncertainty for the rotational temperature and does not significantly affect the column density. The results of the minimisation for these species are indicated in the notes of Table 1. Despite the large frequency range of the survey, $\mathrm{H}_{2} \mathrm{CO}$ isotopologues only have a few lines in the range, and most of them are either optically thick or the transition levels are not populated at all $\left(E_{\text {up }}>1000 \mathrm{~K}\right)$. The rotational temperature was derived using two optically thin lines of $\mathrm{H}_{2}^{13} \mathrm{CO}$, with upper state energies of 98 and $240 \mathrm{~K}$. The optically thin lines, which were used to derive the column density of the other isotopologue, are the same lines used by Persson et al. (2018) in the analysis of IRAS 16293 B.

Similar to the study of IRAS 16293 B (Jørgensen et al. 2018), a large number of rarer isotopologues were also identified towards IRAS $16293 \mathrm{~A}$. We note that $\mathrm{CH}_{3}^{18} \mathrm{OH}$ and $\mathrm{H}_{2} \mathrm{C}^{18} \mathrm{O}$ show a ${ }^{16} \mathrm{O} /{ }^{18} \mathrm{O}$ ratio close to the local interstellar medium value of $557 \pm 30$ (Wilson 1999). The upper limit column density of $\mathrm{H}_{2} \mathrm{C}^{17} \mathrm{O}$ is consistent with the canonical ${ }^{16} \mathrm{O} /{ }^{17} \mathrm{O}$ ratio of $2005 \pm 155$ (Penzias 1981; Wilson 1999). The ${ }^{13} \mathrm{C}$-isotopologues that were detected do not show any significant deviation from the local ISM ${ }^{12} \mathrm{C} /{ }^{13} \mathrm{C}$ ratio of $68 \pm 15$ (Milam et al. 2005). Lower ${ }^{12} \mathrm{C} /{ }^{13} \mathrm{C}$ ratios have been reported towards IRAS $16293 \mathrm{~B}$ (Jørgensen et al. 2018) and were interpreted by a chemical process that is similar to the deuteration enhancement on the ice grain surface, which occurs during the pre-stellar formation stage. However, the mean ${ }^{12} \mathrm{C} /{ }^{13} \mathrm{C}$ ratio of $76 \pm 12$ towards IRAS $16293 \mathrm{~A}$ is consistent with the local ISM value, which indicates that the isotopic enhancement mechanism observed in IRAS $16293 \mathrm{~B}$ does not occur in IRAS $16293 \mathrm{~A}$. Deuterated isotopologues of $\mathrm{CH}_{3} \mathrm{OH}, \mathrm{H}_{2} \mathrm{CO}, \mathrm{C}_{2} \mathrm{H}_{5} \mathrm{OH}$, dimethyl ether $\left(\mathrm{CH}_{3} \mathrm{OCH}_{3}\right), \mathrm{CH}_{3} \mathrm{OCHO}, \mathrm{CH}_{2} \mathrm{CO}, \mathrm{HCOOH}$, and isocyanic acid ( $\mathrm{HNCO}$ ) are present in the gas with $\mathrm{D} / \mathrm{H}$ ratios of $2-5 \%$. As it was observed towards IRAS 16293 B (Persson et al. 2018; Manigand et al. 2019), the doubly-deuterated isotopologues $\mathrm{D}_{2} \mathrm{CO}$ and $\mathrm{CHD}_{2} \mathrm{OCHO}$ are significantly enhanced compared to the singly-deuterated isotopologues. The implications are discussed in the next section of the paper.

New spectroscopic data for deuterated $\mathrm{CH}_{3} \mathrm{CHO}$ isotopologues were recently reported by Coudert et al. (2019) who add information about $\mathrm{CH}_{2}$ DCHO to that of $\mathrm{CH}_{3} \mathrm{CDO}$ from Elkeurti et al. (2010), which was utilised for the first detection reported by Jørgensen et al. (2018). To test their experimental results,
Coudert et al. (2019) used the new spectroscopic data to search for $\mathrm{CH}_{2} \mathrm{DCHO}$ and $\mathrm{CH}_{3} \mathrm{CDO}$ in the PILS dataset. They report the presence of 93 and 43 transitions of $\mathrm{CH}_{2} \mathrm{DCHO}$ and $\mathrm{CH}_{3} \mathrm{CDO}$ across a $10 \mathrm{GHz}$ frequency range, respectively, and they made rough estimates of the column densities based on the archival $12 \mathrm{~m}$ array PILS data only. In order to carry out a consistent comparison of the PILS results in terms of assumptions concerning analysed positions, source sizes, velocity shift, and FWHM as well as using the combined $12 \mathrm{~m}+\mathrm{ACA}$ dataset, we analysed the data for those isotopologues with the same methodology in this paper as the other PILS papers. The derived column density and rotational temperature are reported in Table 2 . The new column density reported for $\mathrm{CH}_{3} \mathrm{CDO}$ is consistent with the value that was derived in the previous study of Jørgensen et al. (2018).

\subsection{New detections}

In addition to the 31 identified isotopologues towards IRAS $16293 \mathrm{~A}$, two new species, $\mathrm{CH}_{3} \mathrm{OCH}_{2} \mathrm{OH}$ and $\mathrm{t}-\mathrm{C}_{2} \mathrm{H}_{5} \mathrm{OCH}_{3}$, were detected towards IRAS $16293 \mathrm{~B}$, exclusively. Only an upper limit column density was derived for the two species towards IRAS $16293 \mathrm{~A}$, assuming the same rotational temperature as for IRAS $16293 \mathrm{~B}$. Column densities, relative abundances to $\mathrm{CH}_{3} \mathrm{OH}$, and upper limits are reported in Table 2. Both species have already been detected in the ISM before; however, this is the first time they were detected in a low mass star-forming region. McGuire et al. (2017) report the detection of $\mathrm{CH}_{3} \mathrm{OCH}_{2} \mathrm{OH}$ towards the high-mass protostellar core NGC 6334I MM1, with a relative abundance of $\sim 0.03$ with respect to $\mathrm{CH}_{3} \mathrm{OH}$. This abundance is significantly higher than those towards IRAS 16293 A and IRAS 16293 B and this is discussed in the next section along with the other O-bearing species. We note that $\mathrm{t}-\mathrm{C}_{2} \mathrm{H}_{5} \mathrm{OCH}_{3}$ was tentatively detected in several high-mass sources, such as W51 e1/e2 (Fuchs et al. 2005), which was refuted by Carroll et al. (2015) and Orion KL (Tercero et al. 2015). Later, Tercero et al. (2018) confirmed the detection towards the Orion KL compact bridge region. The last study reported a rotational temperature of $150 \pm 20 \mathrm{~K}$ and a column density of $3.0 \pm 0.9 \times 10^{15} \mathrm{~cm}^{-2}$, leading to an abundance of $3.7 \times 10^{-9}$ with respect to $\mathrm{H}_{2}$. This estimation is consistent with the abundance derived towards IRAS 16293 A and B.

\subsection{Identification of $\mathrm{CHD}_{2} \mathrm{OH}$ lines}

Thirty-one lines of $\mathrm{CHD}_{2} \mathrm{OH}$ were found in the range of the observations. $\mathrm{CHD}_{2} \mathrm{OH}$ has already been detected at lower frequencies towards IRAS 16293 (Parise et al. 2002) using the IRAM 30-metre telescope. At the present day, only a small number of lines are publicly available in terms of spectroscopic data, including the line strength values, although, more extended frequency lists can be found in the literature (e.g. Ndao et al. 2016; Mukhopadhyay 2016). Therefore, this species requires special treatment.

To confirm the identification $\mathrm{CHD}_{2} \mathrm{OH}$ in spectrum, each line was independently fitted to a simple Gaussian line profile in terms of amplitude and by using the same velocity peak $v_{\mathrm{lsr}}$ and FWHM as $\mathrm{CH}_{3} \mathrm{OH}$, that is, 0.8 and $2.2 \mathrm{~km} \mathrm{~s}^{-1}$ as well as 2.7 and $1.0 \mathrm{~km} \mathrm{~s}^{-1}$ for IRAS $16293 \mathrm{~A}$ and IRAS $16293 \mathrm{~B}$, respectively. The line frequencies match 24 and 16 lines of the 31 candidates, for IRAS $16293 \mathrm{~A}$ and B, respectively, without any hint of blending effect with other species. Figures D.1 and D.2 show the Gaussian fits of all the lines. The integrated intensity over \pm FWHM around the rest frequency was derived for each unblended lines and is noted in Table D.1. The average 
Table 1. Best-fit results of all the detected and tentatively detected species towards IRAS 16293 A.

\begin{tabular}{|c|c|c|c|c|c|c|}
\hline Species & $\#_{\text {lines }}$ & $\begin{array}{c}E_{\text {up }} \text { range } \\
(\mathrm{K})\end{array}$ & $\begin{array}{l}T_{\text {rot }} \\
(\mathrm{K})\end{array}$ & $\begin{array}{c}N_{\text {tot }} \\
\left(\mathrm{cm}^{-2}\right)\end{array}$ & $N_{\text {tot }} / N_{\mathrm{CH}_{3} \mathrm{OH}}$ & Isotopic ratio $^{(a)}$ \\
\hline $\mathrm{CH}_{3} \mathrm{OH}$ & 18 & $61-1201$ & $130 \pm 26$ & $1.3 \pm 0.4 \times 10^{19}$ & & \\
\hline $\mathrm{CH}_{3}^{18} \mathrm{OH}$ & 7 & $35-338$ & $130 \pm 26^{(b)}$ & $2.3 \pm 0.6 \times 10^{16}$ & $1.8 \pm 0.8 \times 10^{-3}$ & $565 \pm 237$ \\
\hline${ }^{13} \mathrm{CH}_{3} \mathrm{OH}$ & 8 & $329-653$ & $130 \pm 26^{(c)}$ & $2.0 \pm 0.7 \times 10^{17}$ & $1.5 \pm 0.6 \times 10^{-2}$ & $65 \pm 27$ \\
\hline $\mathrm{CH}_{2} \mathrm{DOH}$ & 24 & $61-896$ & $130 \pm 26$ & $1.1 \pm 0.3 \times 10^{18}$ & $8.3 \pm 3.6 \times 10^{-2}$ & $2.8 \pm 0.4 \%$ \\
\hline $\mathrm{CH}_{3} \mathrm{OD}$ & 6 & $104-288$ & $130 \pm 26^{(d)}$ & $2.8 \pm 0.8 \times 10^{17}$ & $2.2 \pm 0.9 \times 10^{-2}$ & $2.2 \pm 0.9 \%$ \\
\hline $\mathrm{CH}_{3} \mathrm{OCHO}^{(\dagger)}$ & 159 & $86-693$ & $115 \pm 23$ & $2.7 \pm 0.8 \times 10^{17}$ & $2.1 \pm 0.9 \times 10^{-2}$ & \\
\hline $\mathrm{CH}_{3} \mathrm{O}^{13} \mathrm{CHO}$ & 6 & $255-308$ & $115 \pm 23^{(\star)}$ & $3.6 \pm 1.1 \times 10^{15}$ & $1.3 \pm 0.5 \times 10^{-2}$ & $75 \pm 32$ \\
\hline $\mathrm{CH}_{2} \mathrm{DOCHO}^{(\dagger)}$ & 82 & $62-417$ & $115 \pm 23$ & $2.3 \pm 0.7 \times 10^{16}$ & $8.5 \pm 3.6 \times 10^{-2}$ & $2.8 \pm 0.4 \%$ \\
\hline $\mathrm{CH}_{3} \mathrm{OCDO}^{(\dagger)}$ & 3 & $282-369$ & $115 \pm 23$ & $4.5 \pm 1.3 \times 10^{16}$ & $1.7 \pm 0.7 \times 10^{-2}$ & $1.7 \pm 0.7 \%$ \\
\hline $\mathrm{CHD}_{2} \mathrm{OCHO}^{(\dagger)}$ & 5 & $266-319$ & $115 \pm 23$ & $5.3 \pm 1.6 \times 10^{15}$ & $2.0 \pm 0.8 \times 10^{-2}$ & $8.2 \pm 0.6 \%$ \\
\hline $\mathrm{CH}_{3} \mathrm{OCH}_{3}$ & 7 & $122-662$ & $100 \pm 20$ & $5.2 \pm 1.6 \times 10^{17}$ & $4.0 \pm 1.7 \times 10^{-2}$ & \\
\hline $\mathrm{CH}_{3} \mathrm{O}^{13} \mathrm{CH}_{3}$ & 11 & $47-234$ & $100 \pm 20$ & $1.2 \pm 0.4 \times 10^{16}$ & $2.3 \pm 1.0 \times 10^{-2}$ & $86 \pm 18$ \\
\hline $\mathrm{s}-\mathrm{CH}_{2} \mathrm{DOCH}_{3}$ & 10 & $46-337$ & $100 \pm 20$ & $2.4 \pm 0.7 \times 10^{16}$ & $4.6 \pm 1.9 \times 10^{-2}$ & $2.3 \pm 0.5 \%$ \\
\hline a- $\mathrm{CH}_{2} \mathrm{DOCH}_{3}$ & 20 & $68-243$ & $100 \pm 20^{(e)}$ & $6.4 \pm 2.0 \times 10^{16}$ & $1.2 \pm 0.5 \times 10^{-1}$ & $3.1 \pm 0.3 \%$ \\
\hline $\mathrm{H}_{2} \mathrm{CO}$ & 2 & $173-(1542)$ & $155 \pm 31^{(\star)}$ & $1.4 \pm 0.4 \times 10^{17}$ & $1.1 \pm 0.5 \times 10^{-2}$ & \\
\hline $\mathrm{H}_{2} \mathrm{C}^{18} \mathrm{O}$ & 3 & $97-279$ & $155 \pm 31^{(\star)}$ & $2.1 \pm 1.0 \times 10^{14}$ & $1.5 \pm 0.6 \times 10^{-3}$ & $667 \pm 280$ \\
\hline $\mathrm{H}_{2}^{13} \mathrm{CO}$ & 4 & $98-240$ & $155 \pm 31$ & $1.5 \pm 0.5 \times 10^{15}$ & $1.1 \pm 0.5 \times 10^{-2}$ & $93 \pm 39$ \\
\hline $\mathrm{H}_{2}^{2} \mathrm{C}^{17} \mathrm{O}$ & 4 & $61-157$ & $155 \pm 31^{(\star)}$ & $<6.0 \times 10^{13}$ & $<4.3 \times 10^{-4}$ & $>2333$ \\
\hline HDCO & 4 & $26-219$ & $155 \pm 31^{(\star)}$ & $6.9 \pm 2.1 \times 10^{15}$ & $4.9 \pm 2.1 \times 10^{-2}$ & $2.5 \pm 0.5 \%$ \\
\hline $\mathrm{D}_{2} \mathrm{CO}$ & 3 & $127-370$ & $155 \pm 31^{(\star)}$ & $5.8 \pm 1.7 \times 10^{15}$ & $4.1 \pm 1.7 \times 10^{-2}$ & $20.2 \pm 4.2 \%$ \\
\hline $\mathrm{C}_{2} \mathrm{H}_{5} \mathrm{OH}$ & 59 & $72-453$ & $135 \pm 27$ & $8.0 \pm 2.4 \times 10^{16}$ & $6.1 \pm 2.6 \times 10^{-3}$ & \\
\hline $\mathrm{CH}_{3} \mathrm{CHDOH}^{(f)}$ & 10 & $55-198$ & $135 \pm 27^{(g)}$ & $7.0 \pm 2.1 \times 10^{15}$ & $8.8 \pm 3.7 \times 10^{-2}$ & $4.4 \pm 0.9 \%$ \\
\hline $\mathrm{CH}_{3} \mathrm{CH}_{2} \mathrm{OD}^{(f)}$ & 9 & $48-192$ & $135 \pm 27^{(\star)}$ & $<3.5 \times 10^{15}$ & $<4.4 \times 10^{-2}$ & $<4.4 \%$ \\
\hline $\mathrm{a}-\mathrm{CH}_{2} \mathrm{DCH}_{2} \mathrm{OH}^{(f)}$ & 9 & $62-205$ & $135 \pm 27^{(\star)}$ & $<3.5 \times 10^{15}$ & $<4.4 \times 10^{-2}$ & $<4.4 \%$ \\
\hline $\mathrm{s}-\mathrm{CH}_{2} \mathrm{DCH}_{2} \mathrm{OH}^{(f)}$ & 7 & $48-199$ & $135 \pm 27^{(\star)}$ & $<5.2 \times 10^{15}$ & $<6.5 \times 10^{-2}$ & $<3.3 \%$ \\
\hline $\mathrm{t}-\mathrm{HCOOH}$ & 8 & $35-530$ & $90 \pm 18$ & $1.3 \pm 0.4 \times 10^{16}$ & $1.0 \pm 0.4 \times 10^{-3}$ & \\
\hline $\mathrm{t}-\mathrm{H}^{13} \mathrm{COOH}$ & 6 & $141-160$ & $90 \pm 18^{(\star)}$ & $<3.3 \times 10^{14}$ & $<2.5 \times 10^{-2}$ & $>39$ \\
\hline t-DCOOH & 7 & $147-225$ & $90 \pm 18^{(\star)}$ & $<5.1 \times 10^{14}$ & $<3.9 \times 10^{-2}$ & $<3.9 \%$ \\
\hline $\mathrm{t}-\mathrm{HCOOD}$ & 6 & $131-208$ & $90 \pm 18^{(\star)}$ & $<2.5 \times 10^{14}$ & $<1.9 \times 10^{-2}$ & $<1.9 \%$ \\
\hline $\mathrm{CH}_{2} \mathrm{CO}$ & 5 & $357-996$ & $135 \pm 27$ & $9.1 \pm 2.7 \times 10^{15}$ & $7.0 \pm 2.9 \times 10^{-4}$ & \\
\hline $\mathrm{CHDCO}$ & 8 & $161-313$ & $135 \pm 27^{(\star)}$ & $<3.6 \times 10^{14}$ & $<4.0 \times 10^{-2}$ & $<2.0 \%$ \\
\hline HNCO & 3 & $333-794$ & $180 \pm 36$ & $1.5 \pm 0.5 \times 10^{16}$ & $1.2 \pm 0.5 \times 10^{-3}$ & \\
\hline DNCO & 5 & $51-366$ & $180 \pm 36^{(\star)}$ & $<2.5 \times 10^{14}$ & $<1.7 \times 10^{-2}$ & $<1.7 \%$ \\
\hline $\mathrm{CH}_{3} \mathrm{CHO}$ & 20 & $153-385$ & $140 \pm 28$ & $3.5 \pm 1.1 \times 10^{15}$ & $2.7 \pm 1.1 \times 10^{-4}$ & \\
\hline $\mathrm{c}-\mathrm{C}_{2} \mathrm{H}_{4} \mathrm{O}$ & 7 & $43-376$ & $95 \pm 19$ & $6.9 \pm 2.1 \times 10^{15}$ & $5.3 \pm 2.2 \times 10^{-4}$ & \\
\hline $\mathrm{CH}_{3} \mathrm{COCH}_{3}$ & 85 & $80-322$ & $125 \pm 25$ & $2.4 \pm 0.7 \times 10^{16}$ & $1.8 \pm 0.8 \times 10^{-3}$ & \\
\hline $\mathrm{CH}_{3} \mathrm{COOH}$ & 17 & $129-407$ & $110 \pm 22$ & $4.5 \pm 1.4 \times 10^{15}$ & $3.5 \pm 1.5 \times 10^{-4}$ & \\
\hline $\mathrm{CH}_{2}(\mathrm{OH}) \mathrm{CHO}$ & 9 & $80-323$ & $155 \pm 31$ & $1.3 \pm 0.4 \times 10^{15}$ & $1.0 \pm 0.4 \times 10^{-4}$ & \\
\hline $\mathrm{NH}_{2} \mathrm{CHO}$ & 8 & $151-453$ & $145 \pm 29$ & $1.9 \pm 0.6 \times 10^{15}$ & $1.5 \pm 0.6 \times 10^{-4}$ & \\
\hline $\mathrm{aGg}^{\prime}-\left(\mathrm{CH}_{2} \mathrm{OH}\right)_{2}$ & 20 & $108-363$ & $145 \pm 29$ & $4.4 \pm 1.3 \times 10^{15}$ & $3.4 \pm 1.4 \times 10^{-4}$ & \\
\hline gGg'-( $\left(\mathrm{CH}_{2} \mathrm{OH}\right)_{2}$ & 12 & $97-115$ & $145 \pm 29^{(\star)}$ & $<4.8 \times 10^{15}$ & $<3.7 \times 10^{-4}$ & \\
\hline $\mathrm{C}_{2} \mathrm{H}_{5} \mathrm{CHO}$ & 3 & $297-330$ & $120 \pm 24$ & $<1.2 \times 10^{15}$ & $<9.2 \times 10^{-5}$ & \\
\hline
\end{tabular}

Notes. ${ }^{(a)}$ The $\mathrm{D} / \mathrm{H}$ ratio, including statistics correction, is expressed in $\%$, whereas other isotopic ratios correspond to ${ }^{12} \mathrm{C} /{ }^{13} \mathrm{C}$ or ${ }^{16} \mathrm{O} /{ }^{18} \mathrm{O} .{ }^{(b)} T_{\text {rot }}$ converged to $105 \mathrm{~K}$ and $N_{\text {tot }}=2.2 \pm 0.7 \times 10^{16} \mathrm{~cm}^{-2}$. ${ }^{(c)} T_{\text {rot }}$ converged to $120 \mathrm{~K}$ and $N_{\text {tot }}=2.3 \pm 0.7 \times 10^{17} \mathrm{~cm}^{-2}$. ${ }^{(d)} T_{\text {rot }}$ converged to $105 \mathrm{~K}$ and $N_{\text {tot }}=3.4 \pm 0.7 \times 10^{17} \mathrm{~cm}^{-2} .{ }^{(e)} T_{\text {rot }}$ converged to $115 \mathrm{~K}$ and $N_{\text {tot }}=6.6 \pm 2.0 \times 10^{16} \mathrm{~cm}^{-2}$. ${ }^{(f)}$ Abundances of $\mathrm{C}_{2} \mathrm{H}_{5} \mathrm{OH}$ isotopologues were derived using the anti conformer entries and corrected to take the gauche conformer into account (see spectroscopic data section in Appendix C). ${ }^{(g)} T_{\text {rot }}$ converged to $125 \mathrm{~K}$ and $N_{\text {tot }}=6.6 \pm 2.0 \times 10^{15} \mathrm{~cm}^{-2}$. $^{(b)-(e),(g)}$ The rotational temperature of the main isotopologue was used to derive the column density, despite the convergence to a slightly different $T_{\text {rot }}{ }^{(\star)}$ The rotational temperature did not converge; the main isotopologue value is assumed. References. ${ }^{(\dagger)}$ Manigand et al. (2019).

integrated intensity of the lines assigned to $\mathrm{CHD}_{2} \mathrm{OH}$ is higher than $0.3 \mathrm{Jy} \mathrm{beam}^{-1} \mathrm{~km} \mathrm{~s}^{-1}$ for IRAS $16293 \mathrm{~B}$. Also, several liness towards IRAS 16293 B show a small blue-shifted absorption counterpart, which is a characteristic of infalling motions (Pineda et al. 2012), which are associated with optically thick lines. In comparison to the other detected species, especially to $\mathrm{CH}_{2} \mathrm{DOH}$ and $\mathrm{CH}_{3} \mathrm{OH}$, these intensities correspond to high line opacities due to a high column density. This suggests a high abundance of $\mathrm{CHD}_{2} \mathrm{OH}$ as well, and it is a key factor motivating further searches for $\mathrm{CD}_{3} \mathrm{OH}$, as was already detected by 


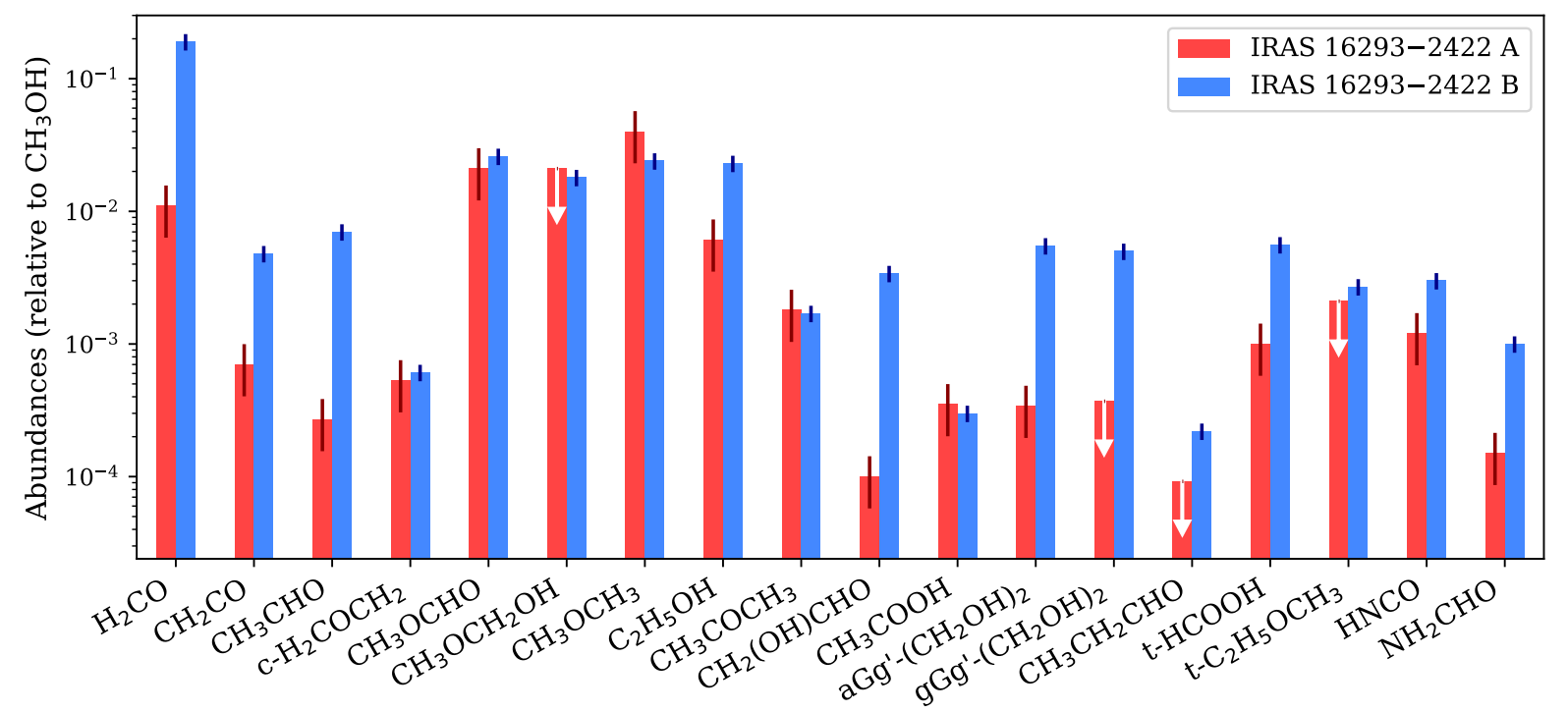

Fig. 2. Relative abundances of the main isotopologues with respect to $\mathrm{CH}_{3} \mathrm{OH}$, towards IRAS $16293 \mathrm{~A}$ in red and IRAS $16293 \mathrm{~B}$ in blue. The abundances towards IRAS $16293 \mathrm{~B}$ are based on Persson et al. (2018) for $\mathrm{H}_{2} \mathrm{CO}$, Lykke et al. (2017) for $\mathrm{CH}_{3} \mathrm{CHO}_{2} \mathrm{c}_{-} \mathrm{H}_{2} \mathrm{COCH}_{2}$, and $\mathrm{CH}_{3} \mathrm{COCH}_{3}$, Jørgensen et al. (2016) for $\mathrm{CH}_{2}(\mathrm{OH}) \mathrm{CHO}$ and $\mathrm{CH}_{3} \mathrm{COOH}$, and Jørgensen et al. (2018) for $\mathrm{CH}_{2} \mathrm{CO}, \mathrm{CH}_{3} \mathrm{OCH}_{3}, \mathrm{C}_{2} \mathrm{H}_{5} \mathrm{OH}, \mathrm{CH}_{3} \mathrm{OCHO},\left(\mathrm{CH} \mathrm{OH}_{2}\right.$, and $\mathrm{t}-\mathrm{HCOOH}$. We note that 30 and $10 \%$ of the uncertainties on the column density are considered for IRAS 16293 A and IRAS 16293 B, respectively.

Table 2. Rotational temperature, column density, and relative abundance with respect to $\mathrm{CH}_{3} \mathrm{OH}$ for the newly detected species.

\begin{tabular}{|c|c|c|c|}
\hline \multirow{2}{*}{ Species } & \multicolumn{3}{|c|}{ IRAS 16293 B } \\
\hline & $T_{\text {rot }}(\mathrm{K})$ & $N_{\text {tot }}\left(\mathrm{cm}^{-2}\right)$ & $N_{\text {tot }} / N_{\mathrm{CH}_{3} \mathrm{OH}}$ \\
\hline $\mathrm{t}-\mathrm{C}_{2} \mathrm{H}_{5} \mathrm{OCH}_{3}$ & 100 & $1.8 \pm 0.2 \times 10^{16}$ & $1.8 \times 10^{-3}$ \\
\hline $\mathrm{CH}_{3} \mathrm{OCH}_{2} \mathrm{OH}$ & 130 & $1.4 \pm 0.2 \times 10^{17}$ & $1.4 \times 10^{-2}$ \\
\hline $\mathrm{CH}_{3} \mathrm{CDO}^{(\dagger)}$ & $125^{(\star)}$ & $7.4 \pm 0.7 \times 10^{15}$ & $7.4 \times 10^{-4}$ \\
\hline $\mathrm{CH}_{2} \mathrm{DCHO}^{(\dagger)}$ & $125^{(\star)}$ & $6.2 \pm 0.6 \times 10^{15}$ & $6.2 \times 10^{-4}$ \\
\hline \multirow{2}{*}{ Species } & \multicolumn{3}{|c|}{ IRAS 16293 A } \\
\hline & $T_{\text {rot }}(\mathrm{K})$ & $N_{\text {tot }}\left(\mathrm{cm}^{-2}\right)$ & $N_{\text {tot }} / N_{\mathrm{CH}_{3} \mathrm{OH}}$ \\
\hline $\mathrm{t}-\mathrm{C}_{2} \mathrm{H}_{5} \mathrm{OCH}_{3}$ & $100^{(\star \star)}$ & $<2.7 \times 10^{16}$ & $<2.1 \times 10^{-3}$ \\
\hline $\mathrm{CH}_{3} \mathrm{OCH}_{2} \mathrm{OH}$ & $130^{(\star \star)}$ & $<2.7 \times 10^{17}$ & $<2.1 \times 10^{-2}$ \\
\hline $\mathrm{CH}_{3} \mathrm{CDO}$ & $140^{(\star)}$ & $<6.0 \times 10^{14}$ & $<4.6 \times 10^{-5}$ \\
\hline $\mathrm{CH}_{2} \mathrm{DCHO}$ & $140^{(\star)}$ & $<6.0 \times 10^{14}$ & $<4.6 \times 10^{-5}$ \\
\hline
\end{tabular}

Notes. ${ }^{(\dagger)} \mathrm{CH}_{2} \mathrm{DCHO}$ and $\mathrm{CH}_{3} \mathrm{CDO}$ were initially reported in Coudert et al. (2019). ${ }^{(\star)}$ The rotational temperature is assumed to be the same as the main isotopologue, which is 100 and $125 \mathrm{~K}$ for IRAS $16293 \mathrm{~A}$ and IRAS $16293 \mathrm{~B}$, respectively (Lykke et al. 2017). ${ }^{(\star \star)}$ The rotational temperature did not converge; the same value as IRAS $16293 \mathrm{~B}$ is assumed.

Parise et al. (2004), and $\mathrm{CD}_{3} \mathrm{OD}$. Despite the substantial data available on $\mathrm{CD}_{3} \mathrm{OH}$ and $\mathrm{CD}_{3} \mathrm{OD}$ (Mollabashi et al. 1993; Walsh et al. 1998; Xu et al. 2004; Müller et al. 2006), we did not find any line that corresponds to those of these two species in the present observations.

\section{Discussion}

\subsection{Comparison between IRAS $16293 A$ and $B$}

Most of the species and their isotopologues reported in this study are present towards both IRAS 16293 A and B; however, their abundances are different in a few cases.
Figure 2 shows the abundances of the main isotopologues with respect to $\mathrm{CH}_{3} \mathrm{OH}$ towards IRAS $16293 \mathrm{~A}$ and $\mathrm{B}$. The column densities, from which the abundances were derived, were determined by using the spectrum extracted from the offset positions, as described in the previous section for IRAS $16293 \mathrm{~A}$, which were used in previous studies of IRAS $16293 \mathrm{~B}$ (Coutens et al. 2016; Lykke et al. 2017; Jørgensen et al. 2016). Towards IRAS $16293 \mathrm{~A}$, the abundances relative to $\mathrm{CH}_{3} \mathrm{OH}$ of $\mathrm{CH}_{3} \mathrm{OCHO}$, ethylene oxide $\left(\mathrm{c}-\mathrm{C}_{2} \mathrm{H}_{4} \mathrm{O}\right), \mathrm{CH}_{3} \mathrm{OCH}_{3}$, $\mathrm{CH}_{3} \mathrm{COCH}_{3}$, acetic acid $\left(\mathrm{CH}_{3} \mathrm{COOH}\right)$, and $\mathrm{HNCO}$ are similar to the abundance observed towards IRAS $16293 \mathrm{~B}$. On the other hand, the abundances of $\mathrm{CH}_{2} \mathrm{CO}, \mathrm{H}_{2} \mathrm{CO}, \mathrm{CH}_{3} \mathrm{CHO}$ $\mathrm{C}_{2} \mathrm{H}_{5} \mathrm{OH}, \mathrm{CH}_{2}(\mathrm{OH}) \mathrm{CHO},\left(\mathrm{CH}_{2} \mathrm{OH}\right)_{2}$, t- $\mathrm{HCOOH}$, and formamide $\left(\mathrm{NH}_{2} \mathrm{CHO}\right)$ are significantly lower towards IRAS $16293 \mathrm{~A}$ than those towards IRAS $16293 \mathrm{~B}$. This selective differentiation could reflect the differentiation of the species across the two protostars. However, it is not possible to rule out the effects of the $\mathrm{CH}_{3} \mathrm{OH}$ abundance with respect to $\mathrm{H}_{2}$, for which the column density cannot be properly estimated. In addition, considering a single species as a reference for the abundance of all the species can interfere with the relation between the abundance of several species. For example, in the case of IRAS 16293 and the comet 67P/Churyumov-Gerasimenko, Drozdovskaya et al. (2019) show that it is more consistent to discuss the abundance of N-bearing and S-bearing species with respect to $\mathrm{CH}_{3} \mathrm{CN}$ and $\mathrm{CH}_{3} \mathrm{SH}$, respectively.

In order to complete the picture, we estimated the statistical distance $S$ of abundance ratios of each pair of species between IRAS 16293 A and B, as detailed in Appendix A. Figure 3 shows the colour map of this cross-comparison; each cell corresponds to the statistical distance of the abundance ratio of two species towards IRAS 16293 A as compared to IRAS 16293 B. The advantage of this comparative analysis is that the abundances do not rely on a single species. This technique offers a global view of the comparison in terms of molecular abundance between the offset position from the two sources. The first and the tenth rows of the map are shown in the right panels of the figure and correspond to the comparison of abundances relative to $\mathrm{CH}_{3} \mathrm{OH}$ and 

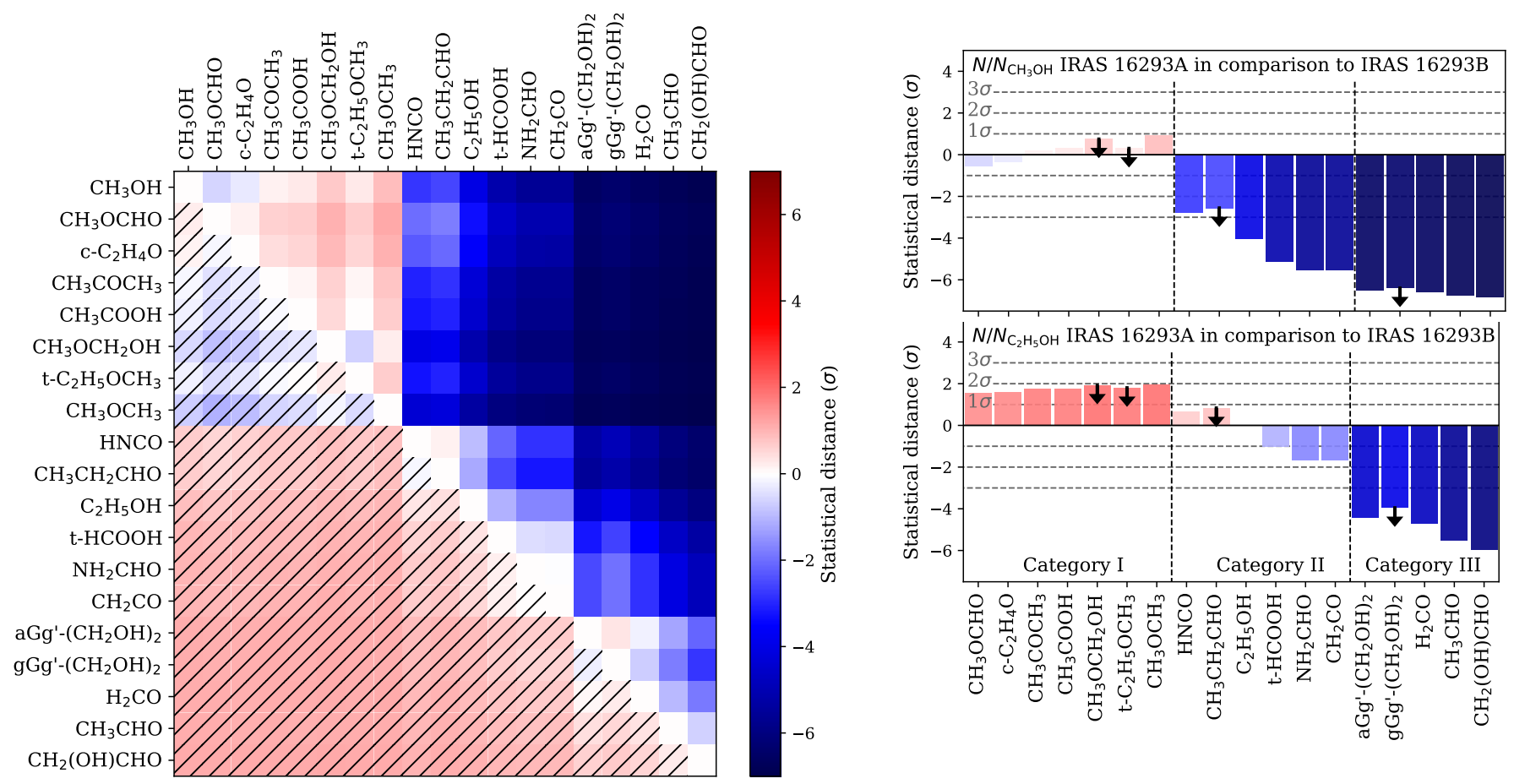

Fig. 3. Left: cross-comparison of species from IRAS 16293 A in comparison to those from IRAS 16293 B. The statistical distance $S$ is represented in the colour scale for each pair of species. One cell with a given value $S$ of the plot should be read as "The abundance of the species $X$ with respect to $Y$ towards IRAS $16293 \mathrm{~A}$ is $S$ sigma higher or lower compared to IRAS $16293 \mathrm{~B}$ ", with $X$ and $Y$ species taken from the $X$-axis and $Y$-axis, respectively. A positive value of $S$ corresponds to $N_{\mathrm{X}} / N_{\mathrm{Y}}$ higher in IRAS 16293 A in comparison to IRAS 16293 B. Right: "CH${ }_{3} \mathrm{OH}$ " and " $\mathrm{C}_{2} \mathrm{H}_{5} \mathrm{OH}$ " rows of the cross-comparison map. The two plots show the comparison of abundances with respect to $\mathrm{CH}_{3} \mathrm{OH}$ (upper panel) and $\mathrm{C}_{2} \mathrm{H}_{5} \mathrm{OH}$ (lower panel) towards IRAS $16293 \mathrm{~A}$ and IRAS $16293 \mathrm{~B}$ in terms of statistical distance.

$\mathrm{C}_{2} \mathrm{H}_{5} \mathrm{OH}$, respectively, towards IRAS $16293 \mathrm{~A}$ in comparison to IRAS $16293 \mathrm{~B}$. The upper right panel shows a clear distinction between the similar abundance of species with respect to $\mathrm{CH}_{3} \mathrm{OH}$ towards IRAS $16293 \mathrm{~A}$ and $\mathrm{B}$ as well as those with a higher abundance towards IRAS $16293 \mathrm{~B}$ in comparison to those towards IRAS $16293 \mathrm{~A}$. This separation is represented with a vertical dashed line, between $\mathrm{CH}_{3} \mathrm{OCH}_{3}$ and $\mathrm{HNCO}$. A similar separation, which is located between $\mathrm{CH}_{2} \mathrm{CO}$ and $\mathrm{H}_{2} \mathrm{CO}$, is shown on the lower right panel where the abundances are plotted with respect to $\mathrm{C}_{2} \mathrm{H}_{5} \mathrm{OH}$ towards IRAS $16293 \mathrm{~A}$ in comparison to the abundances towards IRAS $16293 \mathrm{~B}$. These separations represent a factor of two difference in the relative abundance ratio between IRAS 16293 A and B. This corresponds to a significance of $2 \sigma$, given the column density uncertainties.

The cross-comparison of all the main isotopologues between IRAS 16293 A and B suggests three distinct categories of species. The first category (noted Category I in Fig. 3) includes the species, such as $\mathrm{CH}_{3} \mathrm{OH}, \mathrm{CH}_{3} \mathrm{OCHO}, \mathrm{c}-\mathrm{C}_{2} \mathrm{H}_{4} \mathrm{O}$, $\mathrm{CH}_{3} \mathrm{COCH}_{3}, \mathrm{CH}_{3} \mathrm{COOH}, \mathrm{CH}_{3} \mathrm{OCH}_{2} \mathrm{OH}, \mathrm{t}-\mathrm{C}_{2} \mathrm{H}_{5} \mathrm{OCH}_{3}$, and $\mathrm{CH}_{3} \mathrm{OCH}_{3}$, which have similar abundances between IRAS $16293 \mathrm{~A}$ and $\mathrm{B}$, or a low absolute statistical distance. $\mathrm{H}_{2} \mathrm{CO}$, $\mathrm{CH}_{3} \mathrm{CHO}, \mathrm{CH}_{2}(\mathrm{OH}) \mathrm{CHO}$, and $\left(\mathrm{CH}_{2} \mathrm{OH}\right)_{2}$ show a much lower abundance with respect to all the other species. This corresponds to the righter most part of the distance map (Category III in Fig. 3). The remaining species are in an intermediate category (Category II in Fig. 3) and are characterised by a lower abundance with respect to the first category species and a higher abundance with respect to the most depleted species for IRAS 16293 A in comparison to IRAS 16293 B. This comparative analysis suggests that there are at least two processes that deplete some of the species towards the offset position of IRAS 16293 A compared to the offset position of IRAS 16293 B.
The possible causes of these depletions are discussed in the following sections.

\subsection{Spatial extent}

The spatial distribution of the emission towards IRAS 16293 A and B can be very different depending on the species. The deconvolved source size of the IRAS 16293 B continuum emission is estimated to be 0.'5 (Jørgensen et al. 2016), which is similar to the beam size of the observations. Most of the detected species roughly follow the same distribution as the continuum and have the same source size. Concerning IRAS 16293 A, the continuum emission, as well as the molecular line emission arising from the gas, is more extended in the NE-SW axis where a $6 \mathrm{~km} \mathrm{~s}^{-1}$ velocity gradient was reported (Pineda et al. 2012; Favre et al. 2014; Jørgensen et al. 2016; Oya et al. 2016). Small variations in the spatial distribution of different species towards IRAS 16293 A can be marginally resolved with the beam of PILS observations. However, the velocity gradient, which is associated with the very high line density that is present in the spectrum, makes it very difficult to integrate a single line across the source without contamination from nearby lines of other species.

Calcutt et al. (2018b) developed a new method to map the emission, called the velocity integrated emission map (VINE map), which takes into account the velocity shift of the emitting gas when integrating over the molecular emission. At each pixel, the integration range is then shifted to the peak velocity of the line to be mapped. When there is no large velocity variation across the map, which is the case for IRAS $16293 \mathrm{~B}$ for instance, the VINE map is equivalent to the standard integrated emission map (moment zero map). For IRAS 16293 A, the velocity shift correction at each pixel of the VINE maps is based on 
S. Manigand et al.: Inventory of complex organic molecules towards IRAS 16293-2422 A
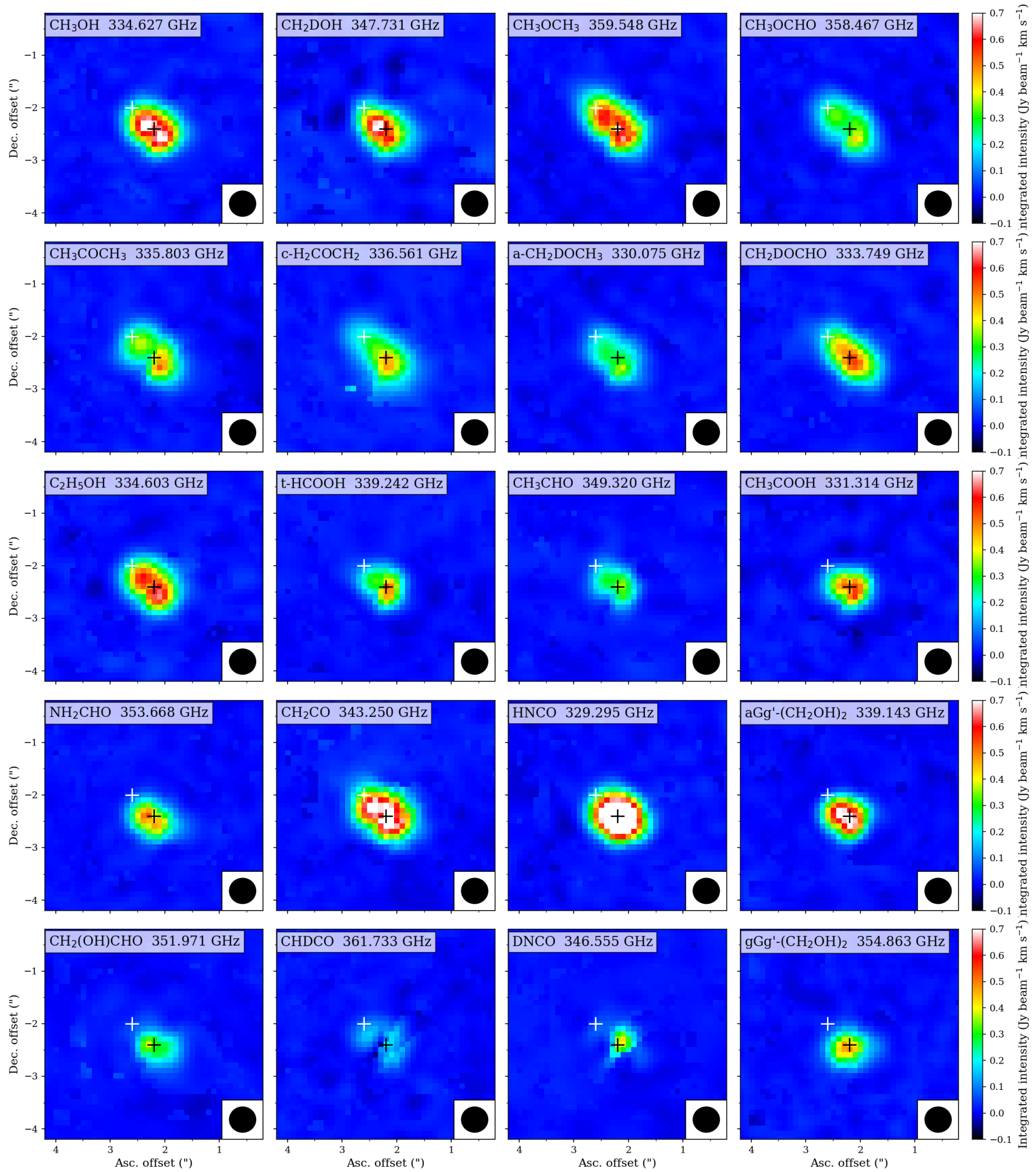

Fig. 4. Representative velocity integrated emission maps of the detected COMs towards IRAS $16293 \mathrm{~A}$. The black and white crosses indicate the continuum peak position and the offset position, respectively. The species and the line frequency are noted in the top-left corner and the 0 '. 5 beam is shown in the bottom right corner of each panels.

the same bright transition $73,5-6_{4,4}$ of $\mathrm{CH}_{3} \mathrm{OH}$ at $337.519 \mathrm{GHz}$ as used by Calcutt et al. (2018b). This transition is particularly suited for tracing the hot corino while being well isolated from other lines. Figure 4 shows VINE maps of the main isotopologues, and few deuterated conformers were detected towards IRAS 16293 A. In this study, the VINE maps were only used to check whether the emission distribution reaches the offset position, where we analysed the spectrum, as indicated by the white cross.

The emission of the species that have similar abundances towards IRAS $16293 \mathrm{~A}$ and B is distributed along the velocity gradient direction and cover the offset position, whereas 
the emission distribution of the depleted species towards IRAS 16293 A in comparison to IRAS 16293 B is located around the continuum peak position of the respective sources and does not show the elliptical distribution of the continuum emission towards IRAS $16293 \mathrm{~A}$. Only the $\mathrm{CH}_{3} \mathrm{COOH}$ emission distribution is as compact as the $\left(\mathrm{CH}_{2} \mathrm{OH}\right)_{2}$ emission distribution, while the abundance towards IRAS 16293 A and B are similar. We checked the distribution emission towards IRAS $16293 \mathrm{~B}$ as well as for this species and noticed that the emission does not cover the offset position used in the previous studies. Therefore, $\mathrm{CH}_{3} \mathrm{COOH}$ seems depleted towards both IRAS $16293 \mathrm{~A}$ and B. This is why $\mathrm{CH}_{3} \mathrm{COOH}$ does not show a difference between IRAS 16293 A and B since it belongs to the most compact region of the warm envelope.

Spatial differences between COMs were previously observed towards the Galactic Centre Sgr B2 region (Hollis et al. 2001) as well as towards high-mass protostars (for example, Calcutt et al. 2014). Hollis et al. (2001) observed the emission of $\mathrm{CH}_{3} \mathrm{OCHO}$ and $\mathrm{CH}_{2}(\mathrm{OH}) \mathrm{CHO}$, among others, at two different angular resolutions. They detected the two species with an $\sim 60^{\prime \prime}$ beam size using the NRAO $12 \mathrm{~m}$ telescope, whereas the Berkeley-Illinois-Maryland Association (BIMA) observations with a beam of $\sim 5^{\prime \prime}$ only revealed $\mathrm{CH}_{3} \mathrm{OCHO}$. They suggested that $\mathrm{CH}_{2}(\mathrm{OH}) \mathrm{CHO}$ was much more extended compared to $\mathrm{CH}_{3} \mathrm{OCHO}$ and its emission was spatially filtered by the interferometric character of the observations. These findings are supported by the more recent study of Li et al. (2017) towards an extended region of the Galactic Centre in which the authors reported cold gas emission of $\mathrm{CH}_{2}(\mathrm{OH}) \mathrm{CHO} \sim 36 \mathrm{pc}$ in width around $\operatorname{Sgr} \mathrm{B} 2(\mathrm{~N})$. We note that, these two observations of the $\mathrm{CH}_{2}(\mathrm{OH}) \mathrm{CHO}$ spatial extent are specific to the Galactic Centre, where non-thermal desorption processes put the $\mathrm{CH}_{2}(\mathrm{OH}) \mathrm{CHO}$ in the gas phase at temperatures much lower than the thermal desorption temperature of this species.

Xue et al. (2019) consistently observed the three isomers $\mathrm{CH}_{3} \mathrm{COOH}, \mathrm{CH}_{3} \mathrm{OCHO}$, and $\mathrm{CH}_{2}(\mathrm{OH}) \mathrm{CHO}$ towards Sgr B2(N) using ALMA in Band 3 with an angular resolution of 1.' 6 , which is sufficient to resolve the sources. The spatial distribution of the species reveals that $\mathrm{CH}_{3} \mathrm{OCHO}$ is more extended than both $\mathrm{CH}_{2}(\mathrm{OH}) \mathrm{CHO}$ and $\mathrm{CH}_{3} \mathrm{COOH}$, which is in agreement with the present study. Xue et al. (2019) suggest that the different formation pathways of the tree species through ice surfaces and gas-phase chemistry could explain the spatial differentiation. This interpretation was supported by the correlation with the distribution of the respective precursors especially for gasphase formation, such as $\mathrm{CH}_{3} \mathrm{OCH}_{3}$, which is the precursor of $\mathrm{CH}_{3} \mathrm{OCHO}$ (Balucani et al. 2015), and $\mathrm{C}_{2} \mathrm{H}_{5} \mathrm{OH}$ the precursor of $\mathrm{CH}_{2}(\mathrm{OH}) \mathrm{CHO}$ and $\mathrm{CH}_{3} \mathrm{COOH}$ (Skouteris et al. 2018). The search for precursors through ice surfaces formation pathway is limited by the current infra-red absorption observations. Alternatively, the authors suggest that the effective desorption temperature of the species could explain their spatial distribution difference based on temperature-programmed desorption (TPD) experiments of $\mathrm{C}_{2} \mathrm{H}_{4} \mathrm{O}_{2}$ isomers (Burke et al. 2015). During the TPD, $\mathrm{CH}_{3} \mathrm{OCHO}$ desorbed at $\sim 70 \mathrm{~K}$ whereas $\mathrm{CH}_{2}(\mathrm{OH}) \mathrm{CHO}$ and $\mathrm{CH}_{3} \mathrm{COOH}$ were released in the gas phase at $\sim 110 \mathrm{~K}$. In the warm-up model of star formation, this would result in a more extended emission of $\mathrm{CH}_{3} \mathrm{OCHO}$ in comparison to the two other isomers.

Calcutt et al. (2014) simultaneously detected $\mathrm{CH}_{3} \mathrm{OCHO}$ and $\mathrm{CH}_{2}(\mathrm{OH}) \mathrm{CHO}$, among other species, towards three high-mass sources, G31.41+0.31, G29.96-0.02, and G24.78+0.08A, which are located at 3.5, 7.1, and $7.7 \mathrm{kpc}$ away, respectively. They find that $\mathrm{CH}_{3} \mathrm{OCHO}$ was more extended than $\mathrm{CH}_{2}(\mathrm{OH}) \mathrm{CHO}$ towards
G31 and that the two species had the same extent towards the two other sources. The spatial differentiation found in $\mathrm{G} 31$ could be similar to IRAS 16293; however, $\mathrm{CH}_{2}(\mathrm{OH}) \mathrm{CHO}$ and the other depleted species towards IRAS 16293 A could also trace a deeper region in the envelope due to the excitation conditions. For example, $\mathrm{CH}_{3} \mathrm{OCHO}$ lines are optically thicker than those of $\mathrm{CH}_{2}(\mathrm{OH}) \mathrm{CHO}$ and show a more extended spatial distribution.

Also, such a spatial differentiation can be inferred indirectly. The first detection of glycolaldehyde by Jørgensen et al. (2012) was performed with ALMA at $\sim 2^{\prime \prime}$ angular resolution: This is enough to separate the two components of the binary system but larger than the source size of both components. Jørgensen et al. (2012) find a relative abundance $\mathrm{CH}_{3} \mathrm{OCHO} / \mathrm{CH}_{2}(\mathrm{OH}) \mathrm{CHO}$ of 10-15 for both protostars on these scales and did not see the same evidence for depletion of $\mathrm{CH}_{2}(\mathrm{OH}) \mathrm{CHO}$ with respect to $\mathrm{CH}_{3}$ OCHO for IRAS $16293 \mathrm{~A}$ in comparison to IRAS $16293 \mathrm{~B}$, as discussed above. However, it is consistent with the interpretation that $\mathrm{CH}_{2}(\mathrm{OH}) \mathrm{CHO}$, as well as the other most depleted species towards the offset position from IRAS $16293 \mathrm{~A}$, are located in a more compact region of the hot corino. This is also supported by observations of other $\mathrm{COMs}$, such as $\mathrm{CH}_{3} \mathrm{OCH}_{3}$, $\mathrm{D}_{2} \mathrm{CO}$ (Jørgensen et al. 2011) and $\mathrm{C}_{2} \mathrm{H}_{5} \mathrm{OH}$ (Bisschop et al. 2008).

\subsection{Differentiation in rotational temperature}

The distribution of COMs towards IRAS 16293 A suggests that a significant part of the emission is missing at the extracted spectrum position. However, the estimation of the rotational temperature is sensitive to the intensity variation of one line with respect to another line with a different upper energy level. Thus, the derivation of the rotational temperature is not affected by the apparent depletion due to the spatial extent and can be securely compared species-to-species.

Towards IRAS $16293 \mathrm{~A}$, the rotational temperatures of the detected species and their isotopologues are between 90 and $180 \mathrm{~K}$, where $\mathrm{HNCO}, \mathrm{H}_{2} \mathrm{CO}, \mathrm{CH}_{2}(\mathrm{OH}) \mathrm{CHO},\left(\mathrm{CH}_{2} \mathrm{OH}\right)_{2}$, $\mathrm{NH}_{2} \mathrm{CHO}, \mathrm{CH}_{3} \mathrm{CHO}$, and $\mathrm{C}_{2} \mathrm{H}_{5} \mathrm{OH}$ are the hottest species. Although the distinction between those hot species and the other species is not pronounced, the hot species seem to be located closer to the protostars compared to the other species, showing a lower rotational temperature.

Using the PILS observations towards IRAS 16293 B, Jørgensen et al. (2018) detected the same species as those presented in this study and derived their rotational temperature. The authors find that the species located in the hot corino could be distinguished into two groups depending on their rotational temperature. Species such as $\mathrm{CH}_{3} \mathrm{OH}, \mathrm{C}_{2} \mathrm{H}_{5} \mathrm{OH}$, $\mathrm{CH}_{3} \mathrm{OCHO}, \mathrm{NH}_{2} \mathrm{CHO}, \mathrm{HNCO}, \mathrm{CH}_{2}(\mathrm{OH}) \mathrm{CHO}$, and $\left(\mathrm{CH}_{2} \mathrm{OH}\right)_{2}$ were observed with an rotational temperature of 250 $300 \mathrm{~K}$, whereas $\mathrm{CH}_{2} \mathrm{CO}, \mathrm{CH}_{3} \mathrm{CHO}, \mathrm{CH}_{3} \mathrm{OCH}_{3}, \mathrm{H}_{2} \mathrm{CO}$, and $\mathrm{c}-\mathrm{H}_{2} \mathrm{COCH}_{2}$ were found at $100-150 \mathrm{~K}$. Using these results and the previous results of PILS observations towards IRAS $16293 \mathrm{~B}$ from Coutens et al. (2016), Jørgensen et al. (2016), and Persson et al. (2018), Jørgensen et al. (2018) find a correlation between the rotational temperature and the desorption temperature of the oxygen-bearing COMs that are thought to mainly form on ice surfaces. This implies that the species that have a high rotational temperature $(250-300 \mathrm{~K})$ are more predominantly located in the hot and compact region of the hot corino, while the lower rotational temperature species belong to a more extended region inside the hot corino (see Fig. 4 of Jørgensen et al. 2018). By comparing this scenario to the difference in the spatial extent observed in IRAS $16293 \mathrm{~A}$, the compact 
species, such as $\mathrm{CH}_{2}(\mathrm{OH}) \mathrm{CHO},\left(\mathrm{CH}_{2} \mathrm{OH}\right)_{2}, \mathrm{NH}_{2} \mathrm{CHO}$, HNCO, and $\mathrm{C}_{2} \mathrm{H}_{5} \mathrm{OH}$, are associated with high rotational temperature, whereas extended species, such as $\mathrm{c}-\mathrm{H}_{2} \mathrm{COCH}_{2}, \mathrm{CH}_{3} \mathrm{OCH}_{3}$, and $\mathrm{CH}_{3} \mathrm{COCH}_{3}$, have a low rotational temperature. The ordering of the species across the onion-like structure of the envelope is in agreement with the desorption temperatures observed in temperature-programmed desorption (TPD) experiments of pure and mixed ices (Öberg et al. 2009; Fedoseev et al. 2015a,b, 2017; Paardekooper et al. 2016; Chuang et al. 2016; Qasim et al. 2019a,b).

In addition, the rotational temperature of $\mathrm{CH}_{3} \mathrm{COOH}$ towards IRAS $16293 \mathrm{~B}$ is consistent with its most compact location in the envelope of both sources. However, the correlation between the rotational temperature and the location does not work in IRAS $16293 \mathrm{~A}$ for $\mathrm{CH}_{3} \mathrm{OH}, \mathrm{CH}_{3} \mathrm{OCHO}, \mathrm{CH}_{2} \mathrm{CO}, \mathrm{H}_{2} \mathrm{CO}$, $\mathrm{CH}_{3} \mathrm{CHO}$, and $\mathrm{t}-\mathrm{HCOOH}$.

Concerning $\mathrm{CH}_{3} \mathrm{OH}$ and $\mathrm{CH}_{3} \mathrm{OCHO}$, these two species were found to be hot species in IRAS $16293 \mathrm{~B}$; however, they correspond to cold and extended species in IRAS 16293 A. Their optically thick lines suggest the presence of two components towards IRAS $16293 \mathrm{~B}$ that are indistinguishable due to the smaller source size with respect to the angular resolution: one is at a rotational temperature of 300 and another is at $\sim 125 \mathrm{~K}$ (Jørgensen et al. 2018). In addition, the rotational temperature derived towards IRAS 16293 A for the same two species is only consistent with the second component at $125 \mathrm{~K}$, indicating that the bulk of the emission consists of the extended warm gas. From this, $\mathrm{CH}_{3} \mathrm{OH}$ and $\mathrm{CH}_{3} \mathrm{OCHO}$ are found to trace not only the central core but also the extended part of the hot corino.

Regarding $\mathrm{CH}_{2} \mathrm{CO}$ isotopologues, there are only a few lines in the range of the observations and most of those are blended with small species, such as ${ }^{33} \mathrm{SO}$ at $\sim 343.08 \mathrm{GHz}$ and $\mathrm{CS}$ at $\sim 342.88 \mathrm{GHz}$. The deuterated isotopologue CHDCO suggests that the low abundance towards IRAS $16293 \mathrm{~A}$ compared to IRAS $16293 \mathrm{~B}$ is due to the spatial extent differences between the two sources. The emission distribution of $\mathrm{H}_{2} \mathrm{CO}$ was found to be much more complex and extended across the binary protostars and it traces both the hot corino region and the outflow interface with the bridge structure between IRAS 16293 A and $\mathrm{B}$ and the E-W outflow emerging from IRAS 16293 A (van der Wiel et al. 2019).

The few unblended, but optically thick, lines of $\mathrm{CH}_{3} \mathrm{CHO}$ show a large distribution towards both sources, suggesting that the bulk of the emission corresponds to the extended part of the hot corino, despite the abundance difference between sources A and B. The three-phase chemical model of Garrod (2013) shows that the abundance of $\mathrm{CH}_{3} \mathrm{CHO}$ in the gas phase increases during the evolution of the hot corino, which occurs even before the species desorbs from the ice grain surfaces. This suggests that gas-phase formation paths significantly contribute to the formation of $\mathrm{CH}_{3} \mathrm{CHO}$ at relatively low temperatures before the bulk of the species desorb from the ice. Concerning the abundance observed towards IRAS 16293 binary, if the gas-phase formation paths effectively produce $\mathrm{CH}_{3} \mathrm{CHO}$ in the outer part of the hot corino, it should increase the abundance of $\mathrm{CH}_{3} \mathrm{CHO}$ towards the most luminous source with respect to the other component. However, the luminosity of IRAS 16293 A was estimated to be $\sim 18 L_{\odot}$, which is six times higher than IRAS 16293 B (Jacobsen et al. 2018).

In summary, the analysis of the spatial extent, the abundances, and the rotational temperatures suggests that the hot corinos show a stratification of the COMs along the distance to the forming star. This spatial differentiation towards IRAS 16293 A could be linked to the rotational temperature of the species

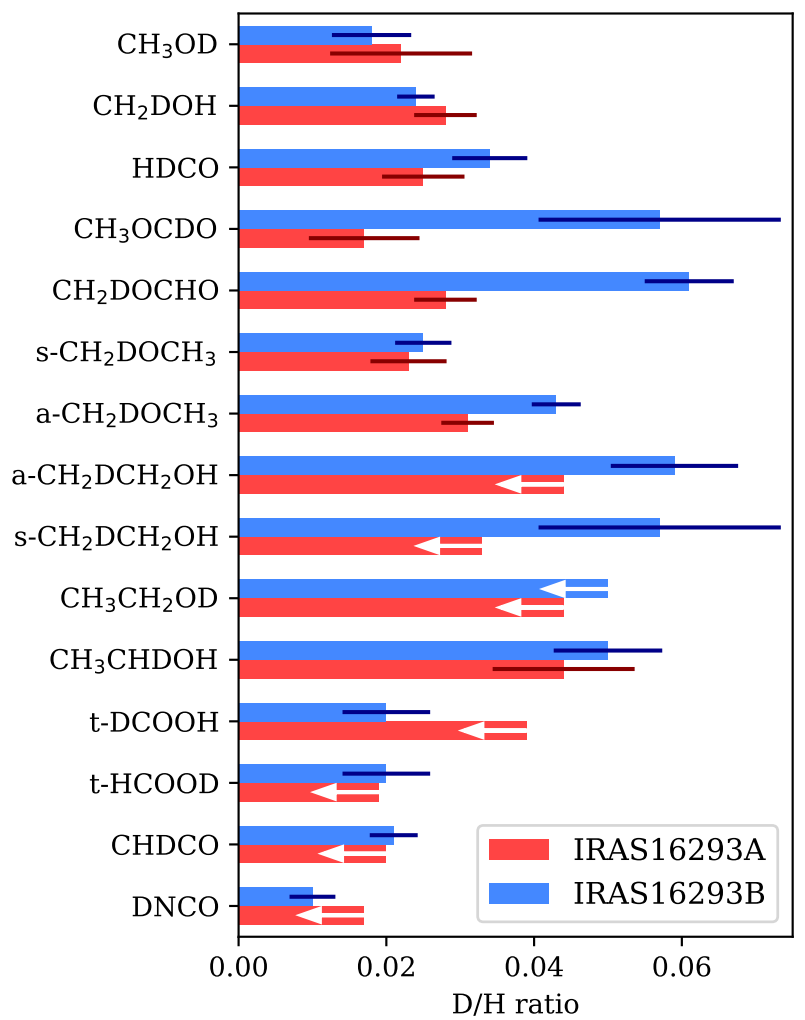

Fig. 5. D/H ratio of the deuterated species detected towards IRAS $16293 \mathrm{~A}$ in red and IRAS $16293 \mathrm{~B}$ in blue. The D/H ratio is calculated from the abundance ratio, with the statistical correction due to the chemical group (see Appendix B of Manigand et al. 2019). D/H ratios towards IRAS $16293 \mathrm{~B}$ are taken from Coutens et al. (2016), Lykke et al. (2017), Persson et al. (2018), and Jørgensen et al. (2018).

observed towards IRAS $16293 \mathrm{~B}$ in the way that species at $300 \mathrm{~K}$ are more compact than the species at $125 \mathrm{~K}$. Although this spatial differentiation is not resolved for IRAS $16293 \mathrm{~B}$, the rotational temperature suggests its presence. Towards IRAS 16293 A, the difference in rotational temperature between compact and extended species is less pronounced, which could be due to the geometry of the source (inclination, size) or the presence of the nearly edge-on disc (Pineda et al. 2012; Favre et al. 2014; Girart et al. 2014).

\section{4. $\mathrm{D} / \mathrm{H}$ ratio}

The sensitivity of the ALMA observations makes the detection of many deuterated isotopologues of the oxygen-bearing species possible. Figure 5 compares the $\mathrm{D} / \mathrm{H}$ ratios of these species derived from the estimated column densities found towards the offset position of IRAS 16293 A and reported in the previous studies of IRAS 16293 B (Coutens et al. 2016; Lykke et al. 2017; Persson et al. 2018; Jørgensen et al. 2018) on a linear scale. In general, the $\mathrm{D} / \mathrm{H}$ ratios of IRAS $16293 \mathrm{~A}$ species are found to be as high as those of the species of IRAS 16293 B. However, due to the higher uncertainty in the column densities and the higher number of upper limits, it is difficult to observe a trend in the deuteration itself. Nevertheless, there is apparently no direct correlation between the $\mathrm{D} / \mathrm{H}$ ratios and the stratification suggested by the rotational temperatures. This could suggest that contrary to the rotational temperatures and the spatial extents, which give a clue as to the structure of the hot corino and thus the warm chemistry in situ, the $\mathrm{D} / \mathrm{H}$ ratio gives information about the history of the envelope during the pre-stellar phase 
when the gas was cold enough to let the $\mathrm{D} / \mathrm{H}$ ratio of the frozen species increase. This is supported by the $\mathrm{D} / \mathrm{H}$ ratio of the doubly-deuterated isotopologues $\mathrm{D}_{2} \mathrm{CO}$ of $2.0 \pm 0.4 \times 10^{-1}$ and $\mathrm{CHD}_{2} \mathrm{OCHO}$ of $8.2 \pm 0.6 \times 10^{-2}$ (Manigand et al. 2019) found towards IRAS 16293 A.

Using the result reported in Table 2 and the column density of $\mathrm{CH}_{3} \mathrm{CHO}$ measured towards IRAS $16293 \mathrm{~B}$ (Lykke et al. 2017; Jørgensen et al. 2018), which is $1.2 \times 10^{17} \mathrm{~cm}^{-2}$, the D/H ratio of $\mathrm{CH}_{2} \mathrm{DCHO}$ and $\mathrm{CH}_{3} \mathrm{CDO}$ are 1.4 and $4.7 \%$, respectively. The upper limits of the $\mathrm{D} / \mathrm{H}$ ratio of $\mathrm{CH}_{2} \mathrm{DCHO}$ and $\mathrm{CH}_{3} \mathrm{CDO}$ towards IRAS $16293 \mathrm{~A}$ are $<6$ and $<17 \%$, respectively. The D/H ratio of the groups $\mathrm{CH}_{3}$ - and $\mathrm{HCO}-$ are significantly different for $\mathrm{CH}_{3} \mathrm{CHO}$ even after applying the statistical correction for both sources. Assuming that the main formation route is due to the addition of those two radicals on the ice surface during the warm-up phase, then the difference in deuteration may indicate that the deuteration enhancement is different for $\mathrm{CH}_{3}$ and $\mathrm{HCO}$, which are already in the prestellar phase when the temperature is low enough to favour the deuteration enhancement of $\mathrm{H}_{3}^{+}$in the gas phase. However, the other species thought to be formed on ice surfaces do not show such a difference in the $\mathrm{D} / \mathrm{H}$ ratio between $\mathrm{CH}_{3}-$ or $\mathrm{CH}_{2}-$ and $\mathrm{HCO}-$ groups, such as $\mathrm{CH}_{3} \mathrm{OCHO}$ and $\mathrm{CH}_{2}(\mathrm{OH}) \mathrm{CHO}$ (Jørgensen et al. 2016). This suggests a selective process, independent of the luminosity difference between IRAS $16293 \mathrm{~A}$ and $\mathrm{B}$, which increases the deuteration of $\mathrm{HCO}$ or decreases those of $\mathrm{CH}_{3}$ - without significantly impacting the other species.

As a side note, the high intensity of the candidate lines found for $\mathrm{CHD}_{2} \mathrm{OH}$ in the range of the observations and the enhancement of the $\mathrm{D} / \mathrm{H}$ ratio of the two doubly-deuterated isotopologues $\mathrm{D}_{2} \mathrm{CO}$ and $\mathrm{CHD}_{2} \mathrm{OCHO}$ with respect to their respective singly-deuterated isotopologues, $\mathrm{HDCO}$ and $\mathrm{CH}_{2} \mathrm{DOCHO}$, suggest a roughly equally high column density for $\mathrm{CHD}_{2} \mathrm{OH}$. This stresses the importance of future studies on the spectroscopy of $\mathrm{CHD}_{2} \mathrm{OH}$ and even more its deuterated conformers $\mathrm{CD}_{3} \mathrm{OH}$ and $\mathrm{CD}_{3} \mathrm{OD}$.

\section{Conclusions}

In this study, we analysed the molecular content of the protostar IRAS $16293 \mathrm{~A}$ at the hot corino scale and compared the abundances to those measured towards its protostar companion IRAS 16293 B. Numerous O-bearing species have been detected, along with their rarer isotopologues. The main findings of this work are summarised below.

1. The abundances with respect to $\mathrm{CH}_{3} \mathrm{OH}$ of half of the species are significantly lower towards the $0 .{ }^{\prime} 6$ offset position from IRAS 16293 A in comparison to the 0.5 offset position from IRAS 16293 B in spite of its higher luminosity.

2. The cross-correlation of the main isotopologue abundances highlights a selective differentiation depending on the species observed. Different categories are identified whether the species abundances are similar or significantly different between IRAS $16293 \mathrm{~A}$ and B.

3. The first category, including $\mathrm{CH}_{3} \mathrm{OH}, \mathrm{CH}_{3} \mathrm{OCHO}, \mathrm{c}-\mathrm{C}_{2} \mathrm{H}_{4} \mathrm{O}$, $\mathrm{CH}_{3} \mathrm{COCH}_{3}, \mathrm{CH}_{3} \mathrm{COOH}, \mathrm{CH}_{3} \mathrm{OCH}_{3}, \mathrm{t}-\mathrm{C}_{2} \mathrm{H}_{5} \mathrm{OCH}_{3}$, and $\mathrm{CH}_{3} \mathrm{OCH}_{2} \mathrm{OH}$, corresponds to the species that have a similar abundance towards both IRAS $16293 \mathrm{~A}$ and B. These species show an extended spatial distribution across IRAS 16293 A and have a relatively low rotational temperature of $\sim 125 \mathrm{~K}$ towards IRAS $16293 \mathrm{~B}$, except for $\mathrm{CH}_{3} \mathrm{COOH}, \mathrm{CH}_{3} \mathrm{OH}$ and $\mathrm{CH}_{3} \mathrm{OCHO}$. $\mathrm{CH}_{3} \mathrm{COOH}$ spatial distribution is much more compact towards both IRAS 16293 A and B than the other extended species and the rotational temperature found towards IRAS $16293 \mathrm{~B}$, which is unambiguously $\sim 300 \mathrm{~K}$. In addition, $\mathrm{CH}_{3} \mathrm{OH}$ and $\mathrm{CH}_{3} \mathrm{OCHO}$ show attributes of both compact and extended regions as suggested by their extended distribution and their high desorption temperature. Their emission is suspected to trace both regions in the hot corino.

4. The second category concerns the species showing a significantly lower abundance towards IRAS 16293 A with respect to IRAS $16293 \mathrm{~B}$, which are $\mathrm{HNCO}, \mathrm{C}_{2} \mathrm{H}_{5} \mathrm{CHO}$, $\mathrm{C}_{2} \mathrm{H}_{5} \mathrm{OH}$, t-HCOOH, $\mathrm{NH}_{2} \mathrm{CHO}, \mathrm{CH}_{2} \mathrm{CO}, \mathrm{H}_{2} \mathrm{CO}, \mathrm{CH}_{3} \mathrm{CHO}$, $\mathrm{CH}_{2}(\mathrm{OH}) \mathrm{CHO}$, and $\left(\mathrm{CH}_{2} \mathrm{OH}\right)_{2}$. These species have a more compact spatial distribution towards IRAS 16293 A compared to the first category of species. In addition, they tend to have a high rotational temperature of $\sim 300 \mathrm{~K}$, especially for those that are the most compact, such as $\mathrm{CH}_{2}(\mathrm{OH}) \mathrm{CHO}$ and $\left(\mathrm{CH}_{2} \mathrm{OH}\right)_{2}$. However, the spatial distribution difference is not resolved towards IRAS $16293 \mathrm{~B}$, the rotational temperature difference between compact and extended species is more pronounced though. $\mathrm{H}_{2} \mathrm{CO}, \mathrm{CH}_{2} \mathrm{CO}$, and $\mathrm{CH}_{3} \mathrm{CHO}$ do not fit into any category as they have a roughly compact spatial emission but a low rotational temperature.

5. In the search for O-bearing species, we report the new detection of $\mathrm{t}-\mathrm{C}_{2} \mathrm{H}_{5} \mathrm{OCH}_{3}$ and $\mathrm{CH}_{3} \mathrm{OCH}_{2} \mathrm{OH}$ towards IRAS $16293 \mathrm{~B}$. Their abundances with respect to $\mathrm{CH}_{3} \mathrm{OH}$ are consistent with the previous detection of these species towards the high mass protostellar regions Sgr B2(N) and Orion KL. Upper limits of their column density are derived towards IRAS $16293 \mathrm{~A}$.

6. The $\mathrm{D} / \mathrm{H}$ ratio is not correlated to the structure of the hot corino itself; however, it is the result of what happens during the formation of COMs in the pre-stellar phase. The multiply-deuterated COMs seem to have a systematically higher $\mathrm{D} / \mathrm{H}$ ratio compared to singly-deuterated species. More observations of different sources, supported by future spectroscopic studies of other multiply-deuterated COMs, are required to confirm this trend. In addition, we report the identification of $23 \mathrm{CHD}_{2} \mathrm{OH}$ transitions at $0.8 \mathrm{~mm}$ wavelength, which have not been observed so far. The intensity of $\mathrm{CHD}_{2} \mathrm{OH}$ lines suggests a high abundance and $\mathrm{D} / \mathrm{H}$ ratio, as it is for $\mathrm{CHD}_{2} \mathrm{OCHO}$. The identification of these $\mathrm{CHD}_{2} \mathrm{OH}$ transitions highlights the need for spectroscopic data for $\mathrm{CHD}_{2} \mathrm{OH}$ and $\mathrm{CD}_{3} \mathrm{OH}$ to derive their rotational temperatures and their column densities and to get their $\mathrm{D} / \mathrm{H}$ ratios. As discussed in this paper, abundance variations between IRAS $16293 \mathrm{~A}$ and B suggest that the spatial distributions are different for the species associated with low or high rotational temperatures. Whether the origins of these differences are physical or chemical, future higher angular observations will be critical in resolving the innermost structure of the two protostars.

Acknowledgements. The authors wish to thank the anonymous referee for the constructive comments that significantly improved the paper. The authors are grateful to Brett McGuire and Roman Motiyenko for providing the line strengths of $\mathrm{CH}_{3} \mathrm{OCH}_{2} \mathrm{OH}$ spectroscopic data. The authors acknowledge Gleb Fedoseev for the valuable discussion about the desorption temperatures from the TPD experiments of the O-bearing COMs and Troels C. Petersen for discussions of the statistical methods. This paper makes use of the following ALMA data: ADS/JAO.ALMA\#2012.1.00712.S and ADS/JAO.ALMA\#2013.1.00278.S. ALMA is a partnership of ESO (representing its member states), NSF (USA) and NINS (Japan), together with NRC (Canada), NSC and ASIAA (Taiwan), and KASI (Republic of Korea), in cooperation with the Republic of Chile. The Joint ALMA Observatory is operated by ESO, AUI/NRAO and NAOJ. The group of J.K.J. acknowledges support from the H2020 European Research Council (ERC) (grant agreement No 646908) through ERC Consolidator Grant "S4F". Research at Centre for Star and Planet Formation is funded by the Danish National Research Foundation. A.C. postdoctoral grant is funded by the ERC Starting Grant 3DICE (grant agreement 336474). M.N.D. is supported by the Swiss National Science Foundation (SNSF) Ambizione grant 180079, the 
Center for Space and Habitability (CSH) Fellowship and the IAU Gruber Foundation Fellowship. This research has made use of NASA's Astrophysics Data System and VizierR catalogue access tool, CDS, Strasbourg, France (Ochsenbein et al. 2000), as well as community-developed core Python packages for astronomy and scientific computing including Astropy (Astropy Collaboration 2013), Scipy (Jones et al. 2001), Numpy (van der Walt et al. 2011) and Matplotlib (Hunter 2007).

\section{References}

Altwegg, K., Balsiger, H., Berthelier, J. J., et al. 2017, MNRAS, 469, S130 Andre, P., \& Montmerle, T. 1994, ApJ, 420, 837

Astropy Collaboration (Robitaille, T. P., et al.) 2013, A\&A, 558, A33

Auwera, J. V. 1992, J. Mol. Spectr., 155, 136

Bacmann, A., Taquet, V., Faure, A., Kahane, C., \& Ceccarelli, C. 2012, A\&A, 541, L12

Balucani, N., Ceccarelli, C., \& Taquet, V. 2015, MNRAS, 449, L16

Baskakov, O. I. 1996, J. Mol. Spectr., 180, 266

Baskakov, O. I., Bürger, H., \& Jerzembeck, W. 1999, J. Mol. Spectr., 193, 33

Baskakov, O. I., Alekseev, E. A., Motiyenko, R. A., et al. 2006, J. Mol. Spectr., 240, 188

Bellet, J., Deldalle, A., Samson, C., Stenbeckeliers, G., \& Wertheimer, R. 1971, J. Mol. Struct., 9, 65

Belov, S. P., Tretyakov, M. Y., Kleiner, I., \& Hougen, J. T. 1993, J. Mol. Spectr., 160,61

Bergner, J. B., Öberg, K. I., Garrod, R. T., \& Graninger, D. M. 2017, ApJ, 841, 120

Bisschop, S. E., Jørgensen, J. K., Bourke, T. L., Bottinelli, S., \& van Dishoeck E. F. 2008 , A\&A, 488, 959

Biver, N., Bockelée-Morvan, D., Debout, V., et al. 2014, A\&A, 566, L5

Blake, G. A., Sutton, E. C., Masson, C. R., \& Phillips, T. G. 1987, ApJ, 315, 621

Blanco, S., López, J. C., Lesarri, A., \& Alonso, J. L. 2006, J. Am. Chem. Soc., 128,12111

Bockelée-Morvan, D., Lis, D. C., Wink, J. E., et al. 2000, A\&A, 353, 1101

Bocquet, R., Demaison, J., Poteau, L., et al. 1996, J. Mol. Spectr., 177, 154

Bocquet, R., Demaison, J., Cosléou, J., et al. 1999, J. Mol. Spectr., 195, 345

Bøgelund, E. G., Barr, A. G., Taquet, V., et al. 2019, A\&A, 628, A2

Botta, O., \& Bada, J. L. 2002, Surv. Geophys., 23, 411

Bouchez, A., Walters, A., Müller, H. S. P., et al. 2012, J. Quant. Spectr. Rad. Transf., 113, 1148

Boussessi, R., Senent, M. L., \& Jaïdane, N. 2016, J. Chem. Phys., 144, 164110

Brown, R. D., Godfrey, P. D., McNaughton, D., Pierlot, A. P., \& Taylor, W. H. 1990, J. Mol. Spectr., 140, 340

Brünken, S., Müller, H. S. P., Lewen, F., \& Winnewisser, G. 2003, Phys. Chem. Chem. Phys, 5, 1515

Burke, D. J., Puletti, F., Brown, W. A., et al. 2015, MNRAS, 447, 1444

Butcher, S. S., \& Bright Wilson, Jr., E. 1964, J. Chem. Phys., 40, 1671

Butler, R. A. H., De Lucia, F. C., Petkie, D. T., et al. 2001, ApJS, 134, 319

Calcutt, H., Viti, S., Codella, C., et al. 2014, MNRAS, 443, 3157

Calcutt, H., Fiechter, M. R., Willis, E. R., et al. 2018a, A\&A, 617, A95

Calcutt, H., Jørgensen, J. K., Müller, H. S. P., et al. 2018b, A\&A, 616, A90

Carroll, P. B., Drouin, B. J., \& Widicus Weaver, S. L. 2010, ApJ, 723, 845

Carroll, P. B., McGuire, B. A., Blake, G. A., et al. 2015, ApJ, 799, 15

Carvajal, M., Kleiner, I., \& Demaison, J. 2010, ApJS, 190, 315

Caux, E., Kahane, C., Castets, A., et al. 2011, A\&A, 532, A23

Cazaux, S., Tielens, A. G. G. M., Ceccarelli, C., et al. 2003, ApJ, 593, L51

Ceccarelli, C., Caselli, P., Fontani, F., et al. 2017, ApJ, 850, 176

Christen, D., \& Müller, H. S. P. 2003, Phys. Chem. Chem. Phys., 5, 3600

Christen, D., Coudert, L. H., Suenram, R. D., \& Lovas, F. J. 1995, J. Mol. Spectr., 172,57

Christen, D., Coudert, L. H., Larsson, J. A., \& Cremer, D. 2001, J. Mol. Spectr., 205,185

Chuang, K.-J., Fedoseev, G., Ioppolo, S., van Dishoeck, E. F., \& Linnartz, H. 2016, MNRAS, 455, 1702

Chuang, K.-J., Fedoseev, G., Qasim, D., et al. 2018, ApJ, 853, 102

Cornet, R. A., \& Winnewisser, G. 1980, J. Mol. Spectr., 80, 438

Coudert, L. H., Margulès, L., Vastel, C., et al. 2019, A\&A, 624, A70

Coutens, A., Jørgensen, J. K., van der Wiel, M. H. D., et al. 2016, A\&A, 590, A1

Coutens, A., Viti, S., Rawlings, J. M. C., et al. 2018, MNRAS, 475, 2016

Coutens, A., Ligterink, N. F. W., Loison, J. C., et al. 2019, A\&A, 623, L13

Creswell, R. A., \& Schwendeman, R. H. 1974, Chem. Phys. Lett., 27, 521

Crovisier, J., Bockelée-Morvan, D., Biver, N., et al. 2004, A\&A, 418, L35

Cunningham, Jr., G. L., Boyd, A. W., Myers, R. J., Gwinn, W. D., \& Le van, W. I. 1951, J. Chem. Phys., 19, 676

Dangoisse, D., Willemot, E., \& Bellet, J. 1978, J. Mol. Spectr., 71, 414

Demaison, J., Dubrulle, A., Boucher, D., Burie, J., \& van Eijck, B. P. 1982, J. Mol. Spectr., 94, 211
Demaison, J., Maes, H., Van Eijck, B. P., Wlodarczak, G., \& Lasne, M. C. 1987, J. Mol. Spectr., 125, 214

Drozdovskaya, M. N., Walsh, C., van Dishoeck, E. F., et al. 2016, MNRAS, 462, 977

Drozdovskaya, M. N., van Dishoeck, E. F., Jørgensen, J. K., et al. 2018, MNRAS, 476, 4949

Drozdovskaya, M. N., van Dishoeck, E. F., Rubin, M., Jørgensen, J. K., \& Altwegg, K. 2019, MNRAS, 490, 50

Durig, J. R., \& Larsen, R. A. 1990, J. Mol. Struct., 238, 195

Durig, J. R., Compton, D. A. C., \& McArver, A. Q. 1980, J. Chem. Phys., 73, 719

Durig, J. R., Deeb, H., Darkhalil, I. D., et al. 2011, J. Mol. Struct., 985, 202

Dzib, S. A., Ortiz-León, G. N., Hernández-Gómez, A., et al. 2018, A\&A, 614, A20

Ehrenfreund, P., Glavin, D. P., Botta, O., Cooper, G., \& Bada, J. L. 2001, Proc. Natl. Acad. Sci., 98, 2138

Elkeurti, M., Coudert, L. H., Medvedev, I. R., et al. 2010, J. Mol. Spectr., 263, 145

Endres, C. P., Drouin, B. J., Pearson, J. C., et al. 2009, A\&A, 504, 635

Endres, C. P., Schlemmer, S., Schilke, P., Stutzki, J., \& Müller, H. S. P. 2016, J. Mol. Spectr., 327, 95

Fabricant, B., Krieger, D., \& Muenter, J. S. 1977, J. Chem. Phys., 67, 1576

Favre, C., Carvajal, M., Field, D., et al. 2014, ApJS, 215, 25

Favre, C., Fedele, D., Semenov, D., et al. 2018, ApJ, 862, L2

Fayolle, E. C., Öberg, K. I., Garrod, R. T., van Dishoeck, E. F., \& Bisschop, S. E. 2015, A\&A, 576, A45

Fayolle, E. C., Öberg, K. I., Jørgensen, J. K., et al. 2017, Nat. Astron., 1, 703

Fedoseev, G., Cuppen, H. M., Ioppolo, S., Lamberts, T., \& Linnartz, H. 2015a, MNRAS, 448, 1288

Fedoseev, G., Ioppolo, S., Zhao, D., Lamberts, T., \& Linnartz, H. 2015b, MNRAS, 446, 439

Fedoseev, G., Chuang, K. J., Ioppolo, S., et al. 2017, ApJ, 842, 52

Fisher, J., Paciga, G., Xu, L.-H., et al. 2007, J. Mol. Spectr., 245, 7

Fuchs, U., Winnewisser, G., Groner, P., De Lucia, F. C., \& Herbst, E. 2003, ApJS, 144,277

Fuchs, G. W., Fuchs, U., Giesen, T. F., \& Wyrowski, F. 2005, A\&A, 444, 521

Fuchs, G. W., Cuppen, H. M., Ioppolo, S., et al. 2009, A\&A, 505, 629

Gardner, F. F., Godfrey, P. D., \& Williams, D. R. 1980, MNRAS, 193, 713

Garrod, R. T. 2013, ApJ, 765, 60

Garrod, R. T., \& Herbst, E. 2006, A\&A, 457, 927

Garrod, R. T., Widicus Weaver, S. L., \& Herbst, E. 2008, ApJ, 682, 283

Girart, J. M., Estalella, R., Palau, A., Torrelles, J. M., \& Rao, R. 2014, ApJ, 780, L11

Groner, P., Albert, S., Herbst, E., \& De Lucia, F. C. 1998, ApJ, 500, 1059

Groner, P., Albert, S., Herbst, E., et al. 2002, ApJS, 142, 145

Groner, P., Herbst, E., De Lucia, F. C., Drouin, B. J., \& Mäder, H. 2006, J. Mol. Struct., 795, 173

Guarnieri, A. 2005, Z. Naturforsch. A, 60, 619

Guarnieri, A., \& Huckaufa, A. 2003, Z. Naturforsch. A, 58, 275

Hardy, J. A., Cox, A. P., Fliege, E., \& Dreizler, H. 1982, Z. Naturforsch. A, 37, 1035

Hayashi, M., \& Adachi, M. 1982, J. Mol. Struct., 78, 53

Hayashi, M., \& Kuwada, K. 1975, J. Mol. Struct., 28, 147

Hays, B. M., \& Widicus Weaver, S. L. 2013, J. Phys. Chem. A, 117, 7142

Herbst, E., \& van Dishoeck, E. F. 2009, ARA\&A, 47, 427

Hirose, C. 1974, ApJ, 189, L145

Hirota, E., Sugisaki, R., Nielsen, C. J., \& Sørensen, G. O. 1974, J. Mol. Spectr., 49,251

Hocking, W. H., Gerry, M. C. L., \& Winnewisser, G. 1975, Can. J. Phys., 53, 1869

Hollis, J. M., Vogel, S. N., Snyder, L. E., Jewell, P. R., \& Lovas, F. J. 2001, ApJ, 554, L81

Hunter, J. D. 2007, Comput. Sci. Eng., 9, 90

Ikeda, M., Duan, Y.-B., Tsunekawa, S., \& Takagi, K. 1998, ApJS, 117, 249

Ilyushin, V. V., Alekseev, E. A., Dyubko, S. F., et al. 2001, J. Mol. Spectr., 205, 286

Ilyushin, V. V., Alekseev, E. A., Dyubko, S. F., \& Kleiner, I. 2003, J. Mol. Spectr., 220,170

Ilyushin, V., Kleiner, I., \& Lovas, F. J. 2008, J. Phys. Chem. Ref. Data, 37, 97

Ilyushin, V. V., Endres, C. P., Lewen, F., Schlemmer, S., \& Drouin, B. J. 2013, J. Mol. Spectr., 290, 31

Jacobsen, S. K., Jørgensen, J. K., van der Wiel, M. H. D., et al. 2018, A\&A, 612, A72

Johns, J. W. C., \& McKellar, A. R. W. 1977, J. Mol. Spectr., 64, 327

Johns, J. W. C., Nemes, L., Yamada, K. M. T., et al. 1992, J. Mol. Spectr., 156, 501

Johnson, H. R., \& Strandberg, M. W. P. 1952, J. Chem. Phys., 20, 687 
Jones, E., Oliphant, T., Peterson, P., et al. 2001, SciPy: Open source scientific tools for Python

Jørgensen, J. K., Bourke, T. L., Nguyen Luong, Q., \& Takakuwa, S. 2011, A\&A, 534, A 100

Jørgensen, J. K., Favre, C., Bisschop, S. E., et al. 2012, ApJ, 757, L4

Jørgensen, J. K., van der Wiel, M. H. D., Coutens, A., et al. 2016, A\&A, 595, A117

Jørgensen, J. K., Müller, H. S. P., Calcutt, H., et al. 2018, A\&A, 620, A170

Kleiner, I., Lovas, F. J., \& Godefroid, M. 1996, J. Phys. Chem. Ref. Data, 25, 1113

Kobayashi, K., Sakai, Y., Tsunekawa, S., et al. 2016, J. Mol. Spectr., 321, 63

Koerber, M., Bisschop, S. E., Endres, C. P., et al. 2013, A\&A, 558, A112

Krisher, L. C., \& Saegebarth, E. 1971, J. Chem. Phys., 54, 4553

Kryvda, A. V., Gerasimov, V. G., Dyubko, S. F., Alekseev, E. A., \& Motiyenko, R. A. 2009, J. Mol. Spectr., 254, 28

Kukolich, S. G., \& Nelson, A. C. 1971, Chem. Phys. Lett., 11, 383

Kurland, R. J., \& Bright Wilson, Jr., E. 1957, J. Chem. Phys., 27, 585

Kuze, H., Kuga, T., \& Shimizu, T. 1982, J. Mol. Spectr., 93, 248

Lapinov, A. V., Golubiatnikov, G. Y., Markov, V. N., \& Guarnieri, A. 2007, Astron. Lett., 33, 121

Lattanzi, V., Walters, A., Drouin, B. J., \& Pearson, J. C. 2008, ApJS, 176, 536

Le Roy, L., Altwegg, K., Balsiger, H., et al. 2015, A\&A, 583, A1

Lerner, R. G., Dailey, B. P., \& Friend, J. P. 1957, J. Chem. Phys., 26, 680

Li, J., Shen, Z., Wang, J., et al. 2017, ApJ, 849, 115

Ligterink, N. F. W., Coutens, A., Kofman, V., et al. 2017, MNRAS, 469, 2219

Lohilahti, J., \& Horneman, V.-M. 2004, J. Mol. Spectr., 228, 1

López-Sepulcre, A., Sakai, N., Neri, R., et al. 2017, A\&A, 606, A121

Lykke, J. M., Coutens, A., Jørgensen, J. K., et al. 2017, A\&A, 597, A53

Maeda, A., Medvedev, I. R., De Lucia, F. C., Herbst, E., \& Groner, P. 2008a, ApJS, 175, 138

Maeda, A., De Lucia, F. C., \& Herbst, E. 2008b, J. Mol. Spectr., 251, 293

Manigand, S., Calcutt, H., Jørgensen, J. K., et al. 2019, A\&A, 623, A69

Marstokk, K.-M., \& Møllendal, H. 1970, J. Mol. Struct., 5, 205

Marstokk, K.-M., \& Møllendal, H. 1973, J. Mol. Struct., 16, 259

Martinache, L., \& Bauder, A. 1989, Chem. Phys. Lett., 164, 657

McGuire, B. A., Shingledecker, C. N., Willis, E. R., et al. 2017, ApJ, 851, L46

McMullin, J. P., Waters, B., Schiebel, D., Young, W., \& Golap, K. 2007, ASP Conf. Ser., 376, 127

Medcraft, C., Thompson, C. D., Robertson, E. G., Appadoo, D. R. T., \& McNaughton, D. 2012, ApJ, 753, 18

Milam, S. N., Savage, C., Brewster, M. A., Ziurys, L. M., \& Wyckoff, S. 2005, ApJ, 634, 1126

Mollabashi, M., Lees, R. M., Xu, L.-H., \& Bakota, M. 1993, Int. J. Infrared Millim. Waves, 14, 2569

Morina, L., Obst, S., Unrath, M., et al. 2019, J. Mol. Spectr., 356, 1

Moskienko, E. M., \& Dyubko, S. F. 1991, Radiophys. Quant. Electron., 34, 181

Motiyenko, R. A., Tercero, B., Cernicharo, J., \& Margulès, L. 2012, A\&A, 548, A71

Motiyenko, R. A., Margulès, L., Despois, D., \& Guillemin, J.-C. 2018, Phys. Chem. Chem. Phys., 20, 5509

Mukhopadhyay, I. 2016, Infrared Phys. Technol., 75, 139

Müller, H. S. P., \& Christen, D. 2004, J. Mol. Spectr., 228, 298

Müller, H. S. P., \& Lewen, F. 2017, J. Mol. Spectr., 331, 28

Müller, H. S. P., Gendriesch, R., Margulès, L., et al. 2000a, Phys. Chem. Chem Phys., 2, 3401

Müller, H. S. P., Gendriesch, R., Lewen, F., \& Winnewisser, G. 2000b, Z. Naturforsch. A, 55

Müller, H. S. P., Winnewisser, G., Demaison, J., Perrin, A., \& Valentin, A. 2000c, J. Mol. Spectr., 200, 143

Müller, H. S. P., Thorwirth, S., Roth, D. A., \& Winnewisser, G. 2001, A\&A, 370 , L49

Müller, H. S. P., Schlöder, F., Stutzki, J., \& Winnewisser, G. 2005, J. Mol. Struct., 742,215

Müller, H. S. P., Xu, L.-H., \& van der Tak, F. 2006, J. Mol. Struct., 795, 114

Müller, H. S. P., Belloche, A., Xu, L.-H., et al. 2016, A\&A, 587, A92

Ndao, M., Kwabia Tchana, F., Coudert, L. H., et al. 2016, J. Mol. Spectr., 326, 136

Niedenhoff, M., Yamada, K. M. T., Belov, S. P., \& Winnewisser, G. 1995, J. Mol Spectr., 174, 151

Nielsen, C. J. 1973, PhD thesis, Københavns Universitet, Denmark

Öberg, K. I., Garrod, R. T., van Dishoeck, E. F., \& Linnartz, H. 2009, A\&A, 504, 891

Öberg, K. I., Furuya, K., Loomis, R., et al. 2015, ApJ, 810, 112

Ochsenbein, F., Bauer, P., \& Marcout, J. 2000, A\&AS, 143, 23

Oldag, F., \& Sutter, D. H. 1992, Z. Naturforsch. A, 47, 527

Ordu, M. H., Zingsheim, O., Belloche, A., et al. 2019, A\&A, 629, A72

Ospina-Zamudio, J., Lefloch, B., Ceccarelli, C., et al. 2018, A\&A, 618, A145
Oya, Y., Sakai, N., López-Sepulcre, A., et al. 2016, ApJ, 824, 88 Paardekooper, D. M., Bossa, J. B., \& Linnartz, H. 2016, A\&A, 592, A67

Pan, J., Albert, S., Sastry, K. V. L. N., Herbst, E., \& De Lucia, F. C. 1998, ApJ, 499, 517

Parise, B., Ceccarelli, C., Tielens, A. G. G. M., et al. 2002, A\&A, 393, L49

Parise, B., Castets, A., Herbst, E., et al. 2004, A\&A, 416, 159

Pearson, J. C., Sastry, K. V. L. N., Winnewisser, M., Herbst, E., \& De Lucia,

F. C. 1995, J. Phys. Chem. Ref. Data, 24, 1

Pearson, J. C., Sastry, K. V. L. N., Herbst, E., \& De Lucia, F. C. 1996, J. Mol. Spectr., 175, 246

Pearson, J. C., Brauer, C. S., \& Drouin, B. J. 2008, J. Mol. Spectr., 251, 394

Pearson, J. C., Yu, S., \& Drouin, B. J. 2012, J. Mol. Spectr., 280, 119

Penzias, A. A. 1981, ApJ, 249, 518

Persson, M. V., Jørgensen, J. K., \& van Dishoeck, E. F. 2012, A\&A, 541, A39

Persson, M. V., Jørgensen, J. K., Müller, H. S. P., et al. 2018, A\&A, 610, A54

Peter, R., \& Dreizler, H. 1965, Z. Naturforsch. A, 20, 301

Pickett, H. M., Poynter, R. L., Cohen, E. A., et al. 1998, J. Quant. Spectr. Rad. Transf., 60, 883

Pineda, J. E., Maury, A. J., Fuller, G. A., et al. 2012, A\&A, 544, L7

Potin, S., Manigand, S., Beck, P., Wolters, C., \& Schmitt, B. 2019, Icarus, submitted

Qasim, D., Fedoseev, G., Chuang, K. J., et al. 2019a, A\&A, 627, A1

Qasim, D., Lamberts, T., He, J., et al. 2019b, A\&A, 626, A118

Richard, C., Margulès, L., Caux, E., et al. 2013, A\&A, 552, A117

Senent, M., Ruiz, R., Villa, M., \& Domínguez-Gómez, R. 2009, J. Chem. Phys., 130, 064101

Skouteris, D., Vazart, F., Ceccarelli, C., et al. 2017, MNRAS, 468, L1

Skouteris, D., Balucani, N., Ceccarelli, C., et al. 2018, ApJ, 854, 135

Skouteris, D., Balucani, N., Ceccarelli, C., et al. 2019, MNRAS, 482, 3567

Sutter, D. H., \& Dreizler, H. 2000, Z. Naturforsch. A, 55, 695

Tabor, W. J. 1957, J. Chem. Phys., 27, 974

Taquet, V., Wirström, E. S., Charnley, S. B., et al. 2017, A\&A, 607, A20

Tercero, B., Cernicharo, J., López, A., et al. 2015, A\&A, 582, L1

Tercero, B., Cuadrado, S., López, A., et al. 2018, A\&A, 620, L6

Tsunekawa, S., Kinai, Y., Kondo, Y., Odashima, H., \& Takagi, K. 2003, Molecules, 8, 103

Tsunekawa, S., Kinai, Y., Kondo, Y., et al. 2011, Toyama Univ. Mol. Spectr. Data, 4

Turner, P. H., \& Cox, A. P. 1976, Chem. Phys. Lett., 42, 84

Turner, P. H., Cox, A. P., \& Hardy, J. A. 1981, J. Chem. Soc. Faraday Trans. 2, 77,1217

Vacherand, J. M., Van Eijck, B. P., Burie, J., \& Demaison, J. 1986, J. Mol. Spectr., 118,355

van der Walt, S., Colbert, S. C., \& Varoquaux, G. 2011, Comput. Sci. Eng., 13, 22

van der Wiel, M. H. D., Jacobsen, S. K., Jørgensen, J. K., et al. 2019, A\&A, 626, A93

van Dishoeck, E. F., Blake, G. A., Jansen, D. J., \& Groesbeck, T. D. 1995, ApJ, 447, 760

van Eijck, B. P., van Opheusden, J., van Schaik, M. M. M., \& van Zoeren, E. 1981, J. Mol. Spectr., 86, 465

van Eijck, B. P., \& van Duijneveldt, F. B. 1983, J. Mol. Spectr., 102, 273

Vorob'eva, E. M., \& Dyubko, S. F. 1994, Radiophys. Quantum Electron., 37, 155

Walsh, M. S., Xu, L.-H., \& Lees, R. M. 1998, J. Mol. Spectr., 188, 85

Walsh, C., Juhász, A., Meeus, G., et al. 2016, ApJ, 831, 200

Walters, A., Schäfer, M., Ordu, M. H., et al. 2015, J. Mol. Spectr., 314, 6

Wellington Davis, R., Robiette, A. G., Gerry, M. C. L., Bjarnov, E., \& Winnemisser, G. 1980, J. Mol. Spectr., 81, 93

Widicus Weaver, S. L., Butler, R. A. H., Drouin, B. J., et al. 2005, ApJS, 158, 188

Willaert, F., Møllendal, H., Alekseev, E., et al. 2006, J. Mol. Struct., 795, 4

Willemot, E., Dangoisse, D., Monnanteuil, N., \& Bellet, J. 1980, J. Phys. Chem. Ref. Data, 9, 59

Wilson, T. L. 1999, Rep. Prog. Phys., 62, 143

Winnewisser, M., Winnewisser, B. P., Stein, M., et al. 2002, J. Mol. Spectr., 216, 259

Wlodarczak, G., \& Demaison, J. 1988, A\&A, 192, 313

Xu, L.-H., \& Lovas, F. J. 1997, J. Phys. Chem. Ref. Data, 26, 17

Xu, L.-H., Walsh, M. S., \& Lees, R. M. 1996, J. Mol. Spectr., 179, 269

Xu, L. H., Müller, H. S. P., van der Tak, F. F. S., \& Thorwirth, S. 2004, J. Mol. Spectr., 228, 220

Xue, C., Remijan, A. J., Burkhardt, A. M., \& Herbst, E. 2019, ApJ, 871, 112

Zakharenko, O., Motiyenko, R. A., Margulès, L., \& Huet, T. R. 2015, J. Mol. Spectr., 317, 41

Zingsheim, O., Müller, H. S. P., Lewen, F., Jørgensen, J. K., \& Schlemmer, S. 2017, J. Mol. Spectr., 342, 125 


\section{Appendix A: Statistical distance}

The statistical distance of the abundance of a species $X$ relative to a species $Y$ between IRAS 16293 A and IRAS 16293 B is calculated as follows:

$S_{X / Y}=\frac{\left(\frac{N_{X}}{N_{Y}}\right)_{\mathrm{A}}-\left(\frac{N_{X}}{N_{Y}}\right)_{\mathrm{B}}}{\sqrt{\sigma_{\mathrm{A}}^{2}+\sigma_{\mathrm{B}}^{2}}}$

in which $\sigma_{\mathrm{A}}$ and $\sigma_{\mathrm{B}}$ are the uncertainties of the column density ratios $\left(\frac{N_{X}}{N_{Y}}\right)_{\mathrm{A}}$ and $\left(\frac{N_{X}}{N_{Y}}\right)_{\mathrm{B}}$, respectively. The value of $S_{X / Y}$ corresponds to the significance of the distance in terms of the number of $\sigma$.

\section{Appendix B: Monte-Carlo error estimation}

Because of the non-linearity of the model used in this study and the non-standard $\chi^{2}$ minimisation method, the uncertainties on the excitation temperature and the column density are not derivable from the covariance matrix, usually calculated with the $\chi^{2}$ minimisation methods. In this case, the Monte Carlo (MC) simulation becomes a convenient tool to estimate the uncertainties from the posterior probability distributions of each parameter of the model. In this section, we describe the bootstrap algorithm, which is a specific class of Monte Carlo simulations, that was tested to estimate the uncertainties of the parameters of the model.

Consider the data fitted as a vector $S$ of $N$ elements, which are the data points:

$\boldsymbol{S}=\left(s_{0}, s_{1}, s_{2}, \cdots\right)$.

A Gaussian noise $\epsilon$ is defined with the same logic:

$\boldsymbol{\epsilon}=\left(\epsilon_{0}, \epsilon_{1}, \epsilon_{2}, \cdots\right)$.

The $\chi^{2}$ minimisation can be represented by a function $F_{\chi^{2}}$ that transforms a data vector $\boldsymbol{S}$ into a parameter vector $\boldsymbol{P}$, which is the best fit parameters of the model for the given data. The inverse function $F^{-1}$ is thus the fitting model, that is, the synthetic spectrum in the present study:

$\boldsymbol{P}=\left(p_{0}, p_{1}, p_{2}, \cdots\right)$

$F_{\chi^{2}}: S \longrightarrow F_{\chi^{2}}(S)=P$

$F^{-1}: \boldsymbol{P} \longrightarrow F^{-1}(\boldsymbol{P})=\tilde{\boldsymbol{S}}$

in which $\tilde{\boldsymbol{S}}$ is the closest synthetic spectrum to the data $\boldsymbol{S}$. The MC method used in this study is a simple application of the Markov-chain MC simulations where: the prior distribution is a group of normally distributed noise $\left\{\boldsymbol{\epsilon}_{i}\right\}_{N_{\mathrm{MC}}}$ of a width equivalent to the uncertainty $\sigma$ of the data, with $N_{\text {MC }}$ the total number of walkers; the sampler is the $\chi^{2}$ minimisation function $F_{\chi^{2}}$; the probability for a walker to jump to a worse position in the parameter space is null; and only the final position of each walker is kept.

In this particular case, the posterior probability distributions are fully dependent on the prior distributions. From the MC simulation, we get a group of parameter vectors:

$$
\begin{aligned}
\mathcal{P} & =\left\{F_{\chi 2}\left(\boldsymbol{S}+\boldsymbol{\epsilon}_{i}\right)\right\}_{N_{\mathrm{MC}}} \\
& =\left\{\left(p_{0}, p_{1}, p_{2}, \cdots\right)_{0},\left(p_{0}, p_{1}, p_{2}, \cdots\right)_{1}, \cdots\right\}_{N_{\mathrm{MC}}} \\
\mathcal{P}^{T} & =\left(\left\{p_{0}\right\}_{N_{\mathrm{MC}}},\left\{p_{1}\right\}_{N_{\mathrm{MC}}},\left\{p_{2}\right\}_{N_{\mathrm{MC}}}, \cdots\right) \\
\mathcal{P}^{T} & =\left(\mathcal{P}_{0}, \mathcal{P}_{1}, \mathcal{P}_{2}, \cdots\right),
\end{aligned}
$$

where $\mathcal{P}_{i}$ is the group of parameters $p_{i}$. The distribution of $\mathcal{P}_{i}$, called the posterior probability distribution of $p_{i}$, gives the needed information for the fitted parameters. It is maximal for the best fit value and the width at half-maximum of each side of the distribution corresponds to the negative and positive uncertainties on $p_{i}$.

\section{Appendix C: Laboratory spectroscopic data}

Most of the analysed species do not assume any excited vibrational or torsional level contributions to the partition function at low rotational temperatures. However, they are all detected at rotational temperatures above $100 \mathrm{~K}$, where the vibrational and torsional contribution can be significant. Thus, it is necessary to detail the spectroscopic dataset used for each species. In this study, a vibrational correction factor $>1.1$ is considered significant and is not applied to the column density derived from the synthetic spectrum fit if it lies below this value.

Spectroscopic data for $\mathrm{CH}_{3}^{18} \mathrm{OH}$ are provided in the Cologne Database of Molecular Spectroscopy ${ }^{2}$ (CDMS, Endres et al. 2016 and Müller et al. 2005, 2001) from the spectroscopic analyses of Fisher et al. (2007) and Ikeda et al. (1998). The entry of ${ }^{13} \mathrm{CH}_{3} \mathrm{OH}$, from the CDMS catalogue, is based on $\mathrm{Xu} \&$ Lovas (1997) and Xu et al. (1996). The dipole moment is assumed to be the same as of the main isotopomer, and the partition function takes only the permanent dipole moment into account. The spectroscopy of $\mathrm{CH}_{2} \mathrm{DOH}$ is taken from the Jet Propulsion Laboratory catalogue $^{3}$ (JPL, Pickett et al. 1998), where the entry is based on Pearson et al. (2012). The frequency list for $\mathrm{CHD}_{2} \mathrm{OH}$ is based on Ndao et al. (2016), with additional transitions in the range of the PILS observations from Mukhopadhyay (2016). The data do not include line strengths or the partition function; however, the frequencies are assigned with uncertainty much lower than the resolution of the observations used in this study.

The spectroscopic data for $\mathrm{CH}_{3} \mathrm{OCHO}$ isotopologues, except for $\mathrm{CH}_{3} \mathrm{O}^{13} \mathrm{CHO}$, are described in Manigand et al. (2019), and references therein, from which the derived column densities for source $\mathrm{A}$ are taken. The $\mathrm{CDMS}$ entry for $\mathrm{CH}_{3} \mathrm{O}^{13} \mathrm{CHO}$ is based on Carvajal et al. (2010), with measured data in the range of the PILS survey from Willaert et al. (2006) and Maeda et al. (2008a,b). The vibrational contribution of $\mathrm{CH}_{3} \mathrm{O}^{13} \mathrm{CHO}$ is already included in the CDMS entry (Favre et al. 2014).

The data for $\mathrm{CH}_{3} \mathrm{OCH}_{3}$ are provided by the CDMS catalogue. The entry is based on Endres et al. (2009) with additional data in the range of our survey from Groner et al. (1998). The mono-deuterated dimethyl ether $\left(\mathrm{CH}_{2} \mathrm{DOCH}_{3}\right)$ exists in two forms, symmetric and asymmetric. The data of both conformers are taken from Richard et al. (2013). The data of $\mathrm{CH}_{3} \mathrm{O}^{13} \mathrm{CH}_{3}$ are taken from Koerber et al. (2013) and from the Vizier Online Data Catalogue, which is from the Centre de Données Astronomiques de Strasbourg ${ }^{4}$ (CDS). The contribution from excited vibrational levels is estimated to be 1.15 at $T_{\text {ex }}=100 \mathrm{~K}$ for the ${ }^{13} \mathrm{C}$ and deuterated isotopologues.

Spectroscopic data for $\mathrm{CH}_{3} \mathrm{CHO}$ can be found in the JPL catalogue. The entry is based on Kleiner et al. (1996), with experimental transition frequencies in the range of our survey from Belov et al. (1993). The CDMS entry for $\mathrm{CH}_{3} \mathrm{CDO}$ is based on Coudert et al. (2019). The fit and the experimental frequencies used are described in Elkeurti et al. (2010), with additional data below $50 \mathrm{GHz}$ taken from Martinache \& Bauder (1989).

\footnotetext{
2 https://cdms.astro.uni-koeln.de/

https://spec.jpl.nasa.gov/

4 http://cdsarc.u-strasbg.fr/
} 
The partition function includes the first three excited torsional mode contributions and is reliable at $140 \mathrm{~K}$. The spectroscopy of the other deuterated isotopologue $\mathrm{CH}_{2} \mathrm{DCHO}$ is also described in Coudert et al. (2019) with additional data taken from Turner \& Cox (1976) and Turner et al. (1981). The contribution of the lowest torsional mode and the small amplitude vibration are included in the partition function. The partition function converged up to about $100 \mathrm{~K}$. Therefore, vibrational corrections are neglected at $140 \mathrm{~K}$.

$\mathrm{C}_{2} \mathrm{H}_{5} \mathrm{OH}$ isotopologues exist under two isomeric forms, anti and gauche, depending on the $\mathrm{OH}$ group torsion. In addition, the gauche conformer has two degenerated states. The extended analysis of the vibrational ground state is provided by Pearson et al. (2008). However, Müller et al. (2016) find that the predicted intensities do not fit the Sgr B2(N2) emission at $3 \mathrm{~mm}$, and they provide a corrected entry in the CDMS catalogue. This entry combines the anti and the gauche conformer transitions and includes data from Pearson et al. $(1995,1996)$ in the range of the PILS survey. ${ }^{13} \mathrm{C}$ and deuterated $\mathrm{C}_{2} \mathrm{H}_{5} \mathrm{OH}$ isotopologues spectroscopic data are taken from corresponding CDMS entries, based on Bouchez et al. (2012) and Walters et al. (2015), respectively. However, these data consider only the anti conformer. The column density has to be multiplied by a factor of $\sim 2.32$ at $T_{\mathrm{ex}}=$ $135 \mathrm{~K}$ to take the gauche conformer into account (Müller et al. 2016; Jørgensen et al. 2018). The contribution from excited vibrational levels is dominated by the two torsional modes of the methyl group (Durig \& Larsen 1990) ${ }^{5}$. The vibrational correction factor is 1.24 for the main isotopologue (Durig et al. 2011) and is applicable for the ${ }^{13} \mathrm{C}$ and deuterated isotopologues.

Spectroscopic data of the $\mathrm{H}_{2} \mathrm{CO}$ isotopologues are taken from the corresponding CDMS entries and are the result of several contributions over the past four decades. $\mathrm{H}_{2} \mathrm{CO}, \mathrm{H}_{2} \mathrm{C}^{18} \mathrm{O}$ and $\mathrm{H}_{2} \mathrm{C}^{17} \mathrm{O}$ entries are based on Müller \& Lewen (2017), the $\mathrm{H}_{2}^{13} \mathrm{CO}$ entry is based on Müller et al. (2000a), and HDCO and $\mathrm{D}_{2} \mathrm{CO}$ entries are based on Zakharenko et al. (2015). Additional data were taken from Brünken et al. (2003), Bocquet et al. (1996), and Cornet \& Winnewisser (1980) for $\mathrm{H}_{2} \mathrm{CO}$ as well as a few transition frequencies of $\mathrm{H}_{2} \mathrm{C}^{18} \mathrm{O}$ and $\mathrm{H}_{2}^{13} \mathrm{CO}$. Müller et al. (2000c) provided the ground state combination differences employed in the spectroscopic analysis of $\mathrm{H}_{2} \mathrm{CO}$. For the two deuterated isotopologues $\mathrm{HDCO}$ and $\mathrm{D}_{2} \mathrm{CO}$, the data were taken from Lohilahti \& Horneman (2004), Bocquet et al. (1999), and Dangoisse et al. (1978). Additional $\mathrm{H}_{2} \mathrm{C}^{18} \mathrm{O}$ transition frequencies were taken from Müller et al. (2000b). The dipole moments were measured by Fabricant et al. (1977) and Johns \& McKellar (1977). The dipole moments of $\mathrm{H}_{2} \mathrm{C}^{17} \mathrm{O}$ and $\mathrm{H}_{2} \mathrm{C}^{18} \mathrm{O}$ are assumed to be the same as the $\mathrm{H}_{2} \mathrm{CO}$ one. The vibration state energies are way too high to significantly affect the partition function at $155 \mathrm{~K}$.

The publicly available JPL entry for $\mathrm{CH}_{3} \mathrm{COCH}_{3}$, also called propanone, is based on Groner et al. (2002), with the rotational partitioning and the line strength calculation. This study used additional data from Oldag \& Sutter (1992), Vacherand et al. (1986), and Peter \& Dreizler (1965) for transition frequencies up to $300 \mathrm{GHz}$. In the present study, we used an updated version of the spectroscopic data, which will be available on CDMS. This entry is based on the study of Ordu et al. (2019), which includes the previous linelists as well as those from the first two torsional excited states taken from Morina et al. (2019) and Groner et al. (2006). The contribution from excited vibrational level $v=1$ is

\footnotetext{
5 It is the combination of four torsional transitions at 244.4 and $231.1 \mathrm{~cm}^{-1}$ of the trans conformer and 490.0 and $453.0 \mathrm{~cm}^{-1}$ of the gauche conformer.
}

taken into account in the harmonic approximation and thus in the partition function.

c- $\mathrm{H}_{2} \mathrm{COCH}_{2}$ data are provided by the CDMS catalogue. The entry was based on experimental data taken from Hirose (1974), Creswell \& Schwendeman (1974), and Pan et al. (1998). Much more additional data from Medcraft et al. (2012) enriched the set, extending the frequency range from $358 \mathrm{GHz}$ to $4 \mathrm{THz}$. The dipole moment is taken from Cunningham et al. (1951). The vibrational correction factor is insignificant at $95 \mathrm{~K}$.

The rotational spectrum of $\mathrm{CH}_{3} \mathrm{COOH}$ was first extensively analysed by Ilyushin et al. (2008), which was based on a fair amount of measured spectroscopic data from 8 to $358 \mathrm{GHz}$ taken from Tabor (1957), Krisher \& Saegebarth (1971), van Eijck et al. (1981), van Eijck \& van Duijneveldt (1983), Demaison et al. (1982), Wlodarczak \& Demaison (1988), and Ilyushin et al. (2001, 2003). The present study uses the recent datafile provided by Ilyushin et al. (2013). This last study considerably extended the number of measured transition frequencies up to $845 \mathrm{GHz}$ and observed the first two torsional excited states. The partition function takes into account these states and more excited torsional up to $v_{\mathrm{t}}=8$ in the vibrational ground state. The low vibration states are too energetically high to make the vibrational correction factor significant at $110 \mathrm{~K}$.

The JPL catalogue provides the line list for $\mathrm{CH}_{2}(\mathrm{OH}) \mathrm{CHO}$. The entry is based on the rotational lines collection of Carroll et al. (2010). Previous laboratory measurements were carried out by Marstokk \& Møllendal (1970, 1973), and Butler et al. (2001) and served as the base for the latest study. The partition function was previously calculated by Widicus Weaver et al. (2005) over a measured frequency range from 72 to $122.5 \mathrm{GHz}$ and included the ground state and three partially populated vibrational states at room temperature. Carroll et al. (2010) find a systematic bias in the lines with $K_{\mathrm{a}} \sim 28-31$ and they corrected iteratively the assignment in the JPL line list.

$\mathrm{NH}_{2} \mathrm{CHO}$ data used in this study are provided by the CDMS catalogue. The dipole moment was measured by Kurland \& Bright Wilson (1957). The observed experimental frequencies are taken from Kukolich \& Nelson (1971), Nielsen (1973), Hirota et al. (1974), Gardner et al. (1980), Moskienko \& Dyubko (1991), Vorob'eva \& Dyubko (1994), Blanco et al. (2006), and Kryvda et al. (2009). The latest study (Motiyenko et al. 2012) considerably extended the frequency range of measured transitions up to $930 \mathrm{GHz}$. The partition function does not include any contributions from the excited vibrational state. However, the lowest vibration state energy of $288 \mathrm{~cm}^{-1}$ leads to the highest vibration correction factor, which is lower than 1.1 at $145 \mathrm{~K}$.

Spectroscopic data of the $\mathrm{HCOOH}$ isotopologues are provided in the CDMS catalogue for $\mathrm{t}-\mathrm{HCOOH}$ and by JPL for $\mathrm{t}-\mathrm{H}^{13} \mathrm{COOH}, \mathrm{t}-\mathrm{DCOOH}$, and $\mathrm{t}-\mathrm{HCOOD}$. The CDMS entry for the main isotopologue is based on Winnewisser et al. (2002) with additional data from previous studies (Auwera 1992; Willemot et al. 1980). The JPL entries are based on the same study of Lattanzi et al. (2008) gathering the analysis of these three isotopologues. Experimental measurements were taken from Bellet et al. (1971) and Lerner et al. (1957) for the three entries, and Wellington Davis et al. (1980) and Winnewisser et al. (2002) only for the $\mathrm{t}-\mathrm{H}^{13} \mathrm{COOH}$ entry. The dipole moment is assumed to be the same as the main isotopologue, which was measured by Kuze et al. (1982). The excited vibrational state, especially $v_{7}$ and $v_{9}$, was also measured for the ${ }^{13} \mathrm{C}$ and deuterated isotopologues in the studies of Baskakov et al. $(1999,2006)$ and Baskakov (1996). Vibration correction factors are neglected at $90 \mathrm{~K}$ for both the main and deuterated species. 
The CDMS entries for $\mathrm{CH}_{2} \mathrm{CO}$ isotopologues are based on Guarnieri \& Huckaufa (2003) and on Guarnieri (2005) for the deuterated isotopologues (CHDCO). Laboratory measurements of the different isotopologues are taken from Johnson \& Strandberg (1952), Brown et al. (1990), Johns et al. (1992), and Sutter \& Dreizler (2000). The dipole moments were calculated by Fabricant et al. (1977). Vibrations contributions are negligible at $135 \mathrm{~K}$ for both isotopologues.

$\left(\mathrm{CH}_{2} \mathrm{OH}\right)_{2}$ has three coupled rotors along the molecular chain. It can exist in a total of ten stable configurations (Christen et al. 2001) based on the orientation of the two OH groups at both ends of the carbon chain. Most of the transitions, especially the low-energy rotational transitions, are emitted from the two lowest fundamental state conformers aGg'- $\left(\mathrm{CH}_{2} \mathrm{OH}\right)_{2}$ and $\mathrm{gGg}^{\prime}-\left(\mathrm{CH}_{2} \mathrm{OH}\right)_{2}$, the second one lying $290 \mathrm{~K}$ above $\mathrm{aGg}^{\prime}$ (Müller \& Christen 2004). The spectroscopic data used here are taken from the CDMS entries. Experimental measurements and extensive analysis were carried out by Christen et al. (1995) and Christen \& Müller (2003) for $\mathrm{aGg}^{\prime}$ and by Christen et al. (2001), and Müller \& Christen (2004) for $\mathrm{gGg}^{\prime}$, up to 370 and $579 \mathrm{GHz}$, respectively. Concerning the $\mathrm{gGg}^{\prime}$ conformer, the line strength and partition function were treated as if the conformer was the lowest conformer energy. The ground state energy of $290 \mathrm{~K}$ contributes to the line strengths by a factor of 2.35 at $145 \mathrm{~K}$ (Müller \& Christen 2004). The vibrational correction factor at $145 \mathrm{~K}$ of 1.75 , which includes the interactions between $\mathrm{gGg}^{\prime}$ and $\mathrm{aGg}^{\prime}$, were calculated in the anharmonic approximation from Boussessi et al. (2016) for both conformers.

$\mathrm{t}-\mathrm{C}_{2} \mathrm{H}_{5} \mathrm{OCH}_{3}$ data were taken from a preliminary CDMS entry. They are based on Fuchs et al. (2003), with significant additional data from Kobayashi et al. (2016) as well as the experimental measurements from Hayashi \& Adachi (1982) and Tsunekawa et al. (2003, 2011). The dipole moment was measured by Hayashi \& Kuwada (1975). Torsional and vibrational transitions were studied by Senent et al. (2009), providing a future basis for determining the contribution of higher vibrational state energies to the partition function. The partition function and the line strength were revised by H. S. P. Müller. With the lowest vibrations at $288 \mathrm{~cm}^{-1}$, the vibrational correction factor is negligible.

Regarding HNCO isotopologues, the spectroscopic data are taken from the CDMS entry for HNCO, and from the JPL entry for DNCO. The CDMS entry is based on the work of Lapinov et al. (2007), using additional data from Kukolich \& Nelson (1971), Hocking et al. (1975), and Niedenhoff et al. (1995). The JPL entry was last updated in 1987 and only includes the frequencies from Hocking et al. (1975). However, the frequency uncertainties are lower than $0.4 \mathrm{MHz}$ in the range of the PILS observations, except for the transitions at 346.027, 346.176, 346.380 , and $346.541 \mathrm{GHz}$. These transitions correspond to very high upper state energies (i.e. $>1000 \mathrm{~K}$ ) and they are not populated in the temperature and density ranges of IRAS 16293; therefore, the spectroscopy is reliable. We note that the HNCO partition function does not take into account the spin multiplicity of the ${ }^{14} \mathrm{~N}$ nucleus. Vibration correction factors are negligible at $180 \mathrm{~K}$ due to the high lowest vibration contributions of 577 and $\sim 460 \mathrm{~cm}^{-1}$ for $\mathrm{HNCO}$ and DNCO, respectively.

$\mathrm{CH}_{3} \mathrm{OCH}_{2} \mathrm{OH}$ spectroscopy has been investigated very recently because of its lower dipole moment, which makes this species more difficult to detect in space. The data used are based on the work of Motiyenko et al. (2018). They acquired the first measurements of rotation transitions between 150 and $460 \mathrm{GHz}$. The line strength and the partition function have not been published yet; however, they have been kindly provided by B. McGuire under the approval of R. A. Motiyenko. Based on the calculated vibrations (harmonic) from Hays \& Widicus Weaver (2013), the vibrational correction factor is 1.55 at $130 \mathrm{~K}$.

The spectroscopic data of $\mathrm{C}_{2} \mathrm{H}_{5} \mathrm{CHO}$ were found on the CDMS database. The CDMS entry is largely based on the recent study of Zingsheim et al. (2017) with additional data taken from Hardy et al. (1982) and Demaison et al. (1987), though they were remeasured in the most recent study. The dipole moment was measured by Butcher \& Bright Wilson (1964). The partition function takes into account the ground vibrational state alone. The vibration correction factor is 1.42 at $120 \mathrm{~K}$ (Durig et al. 1980). 


\section{Appendix D: Identification of $\mathrm{CHD}_{2} \mathrm{OH}$ transitions}

The identified lines of $\mathrm{CHD}_{2} \mathrm{OH}$ are given in this section, in Table D.1. The unblended lines alone were fitted using a Gaussian line profile and plotted in Figs. D.1 and D.2.
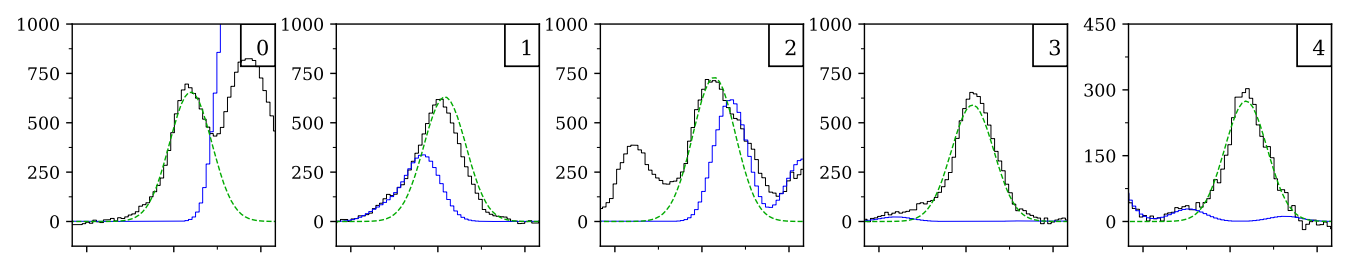

$\begin{array}{lllllllllllllll}330.453 & 330.459 & 330.465 & 330.616 & 330.622 & 330.628 & 331.747 & 331.753 & 331.759 & 332.277 & 332.283 & 332.289 & 332.336 & 332.342 & 332.348\end{array}$
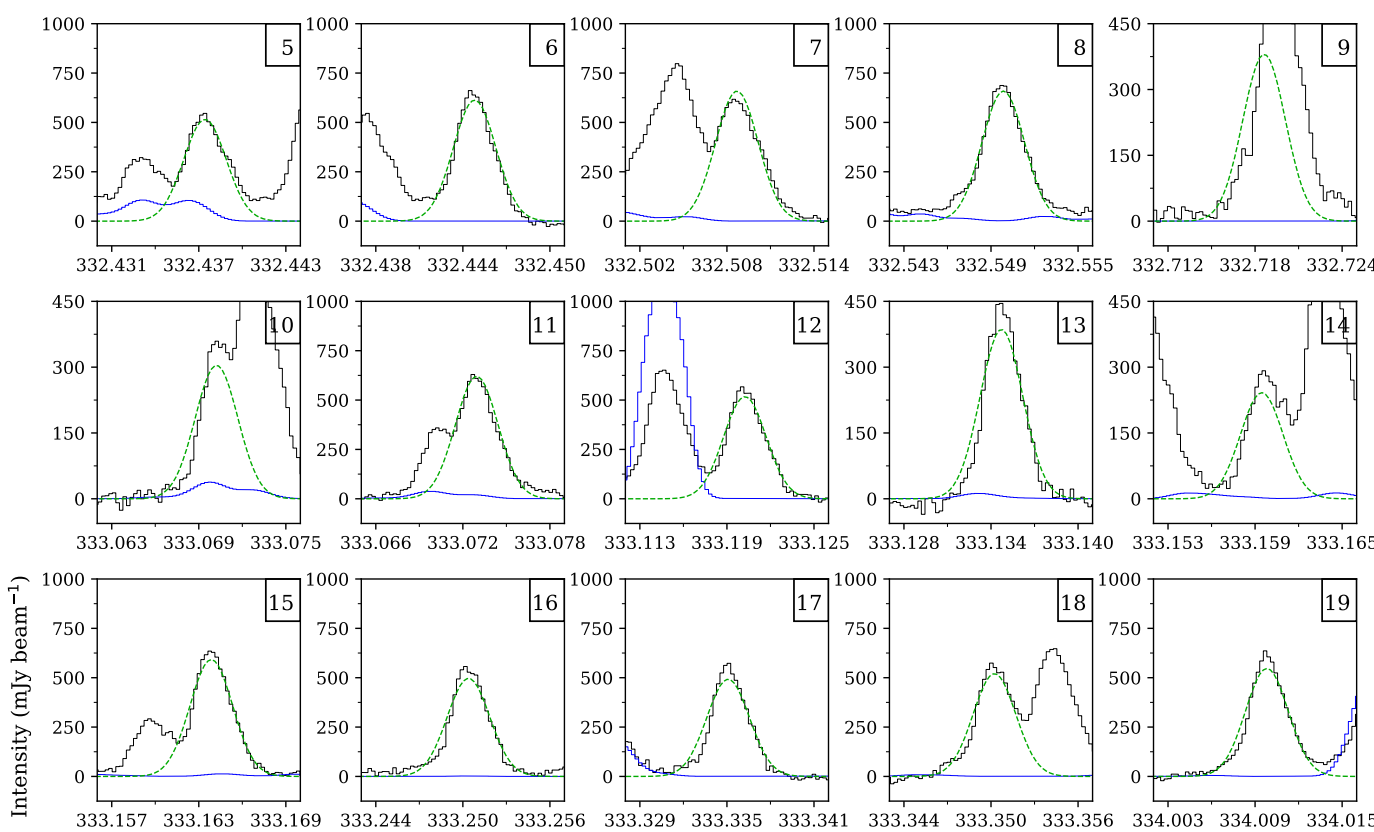

$333.153 \quad 333.159 \quad 333.165$
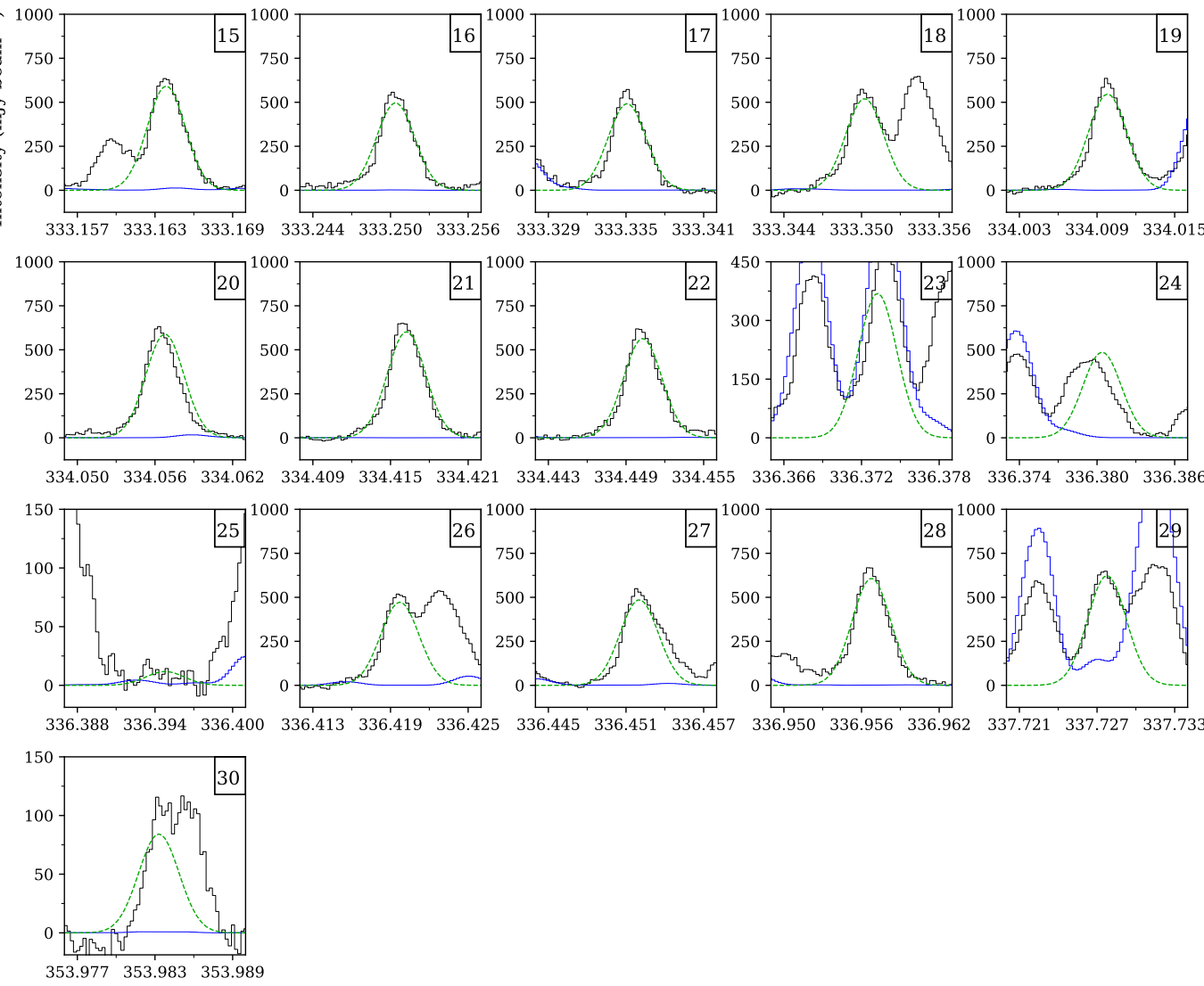

Frequency $(\mathrm{GHz})$

Fig. D.1. Selection of $\mathrm{CHD}_{2} \mathrm{OH}$ identified lines towards IRAS $16293 \mathrm{~A}$ (in black) with the Gaussian fit in green dashed line, with fixed central velocity and FWHM. The same reference spectrum used in the analysis is represented in blue. The squared number in the top-right corner corresponds to the identification number of the transition, i.e. the panel number in Table D.1. 
S. Manigand et al.: Inventory of complex organic molecules towards IRAS 16293-2422 A
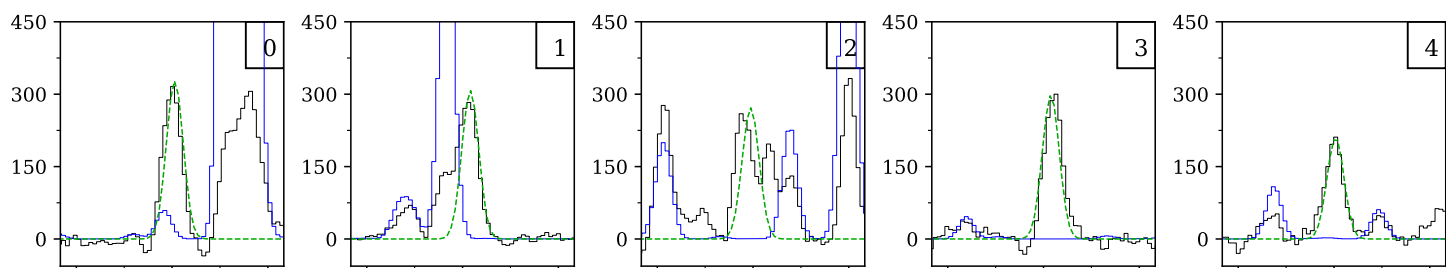

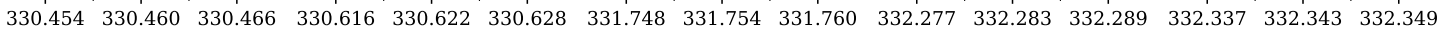
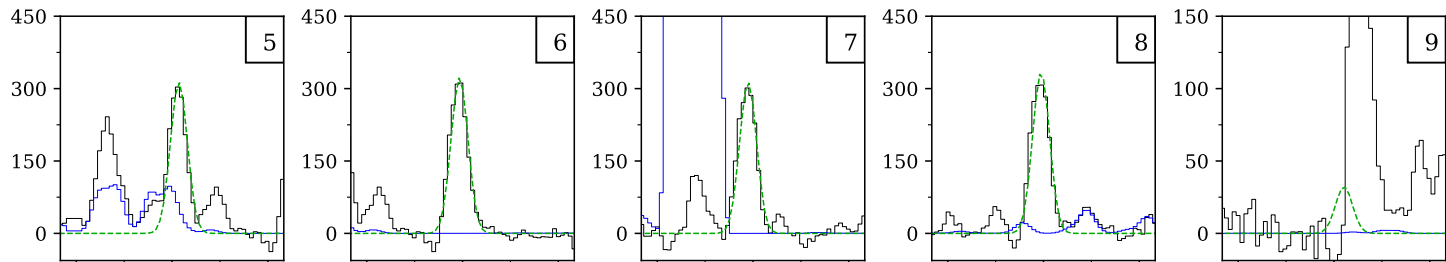

$\begin{array}{llllllllllllllll}332.431 & 332.437 & 332.443 & 332.439 & 332.445 & 332.451 & 332.503 & 332.509 & 332.515 & 332.544 & 332.550 & 332.556 & 332.712 & 332.718 & 332.724\end{array}$
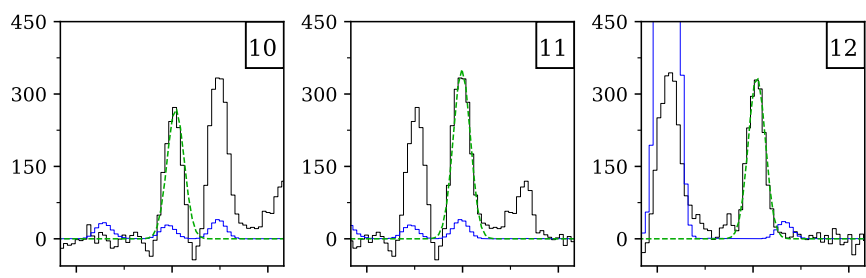

$2{ }^{450} \longrightarrow 13$

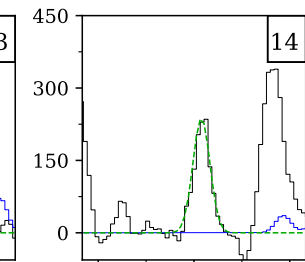

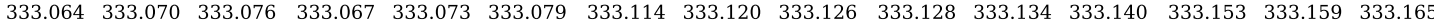
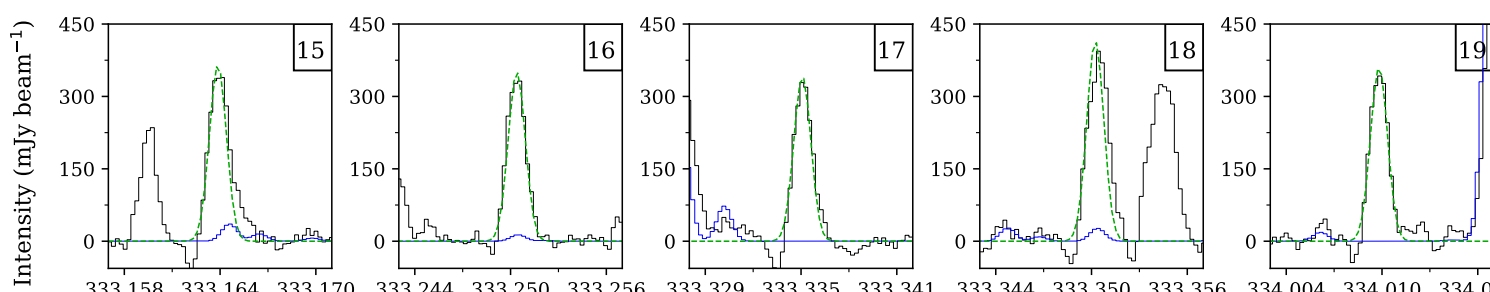

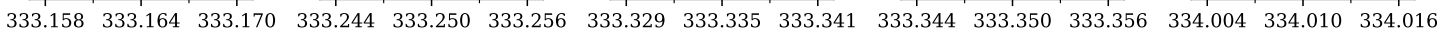
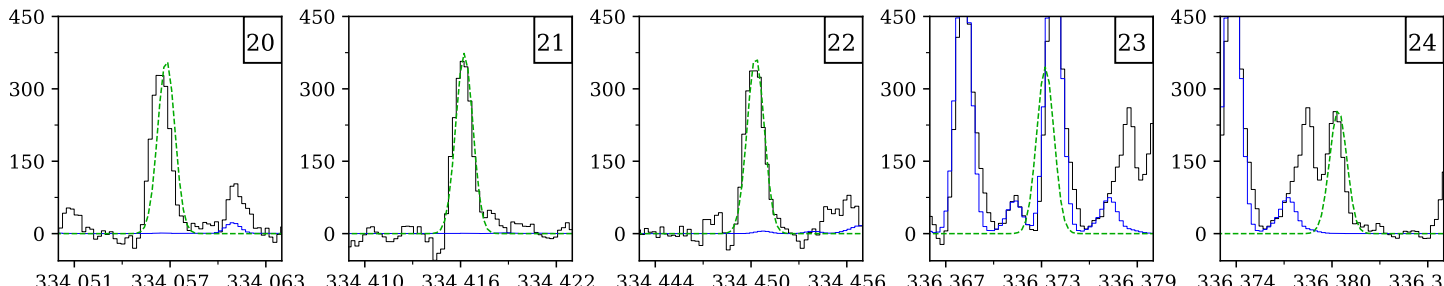

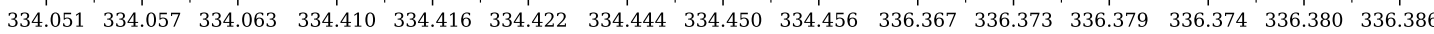
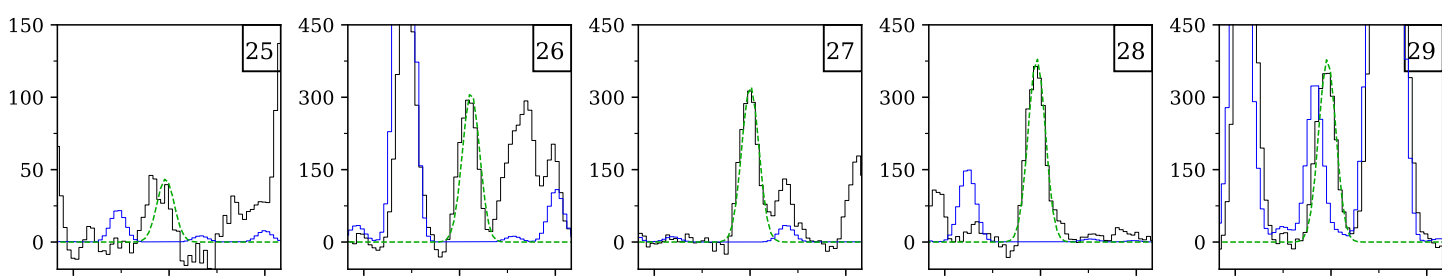

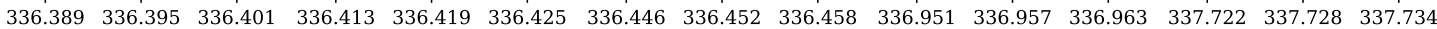

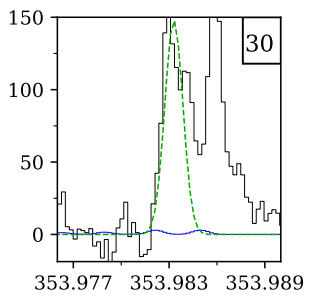

Frequency $(\mathrm{GHz})$

Fig. D.2. Selection of $\mathrm{CHD}_{2} \mathrm{OH}$ identified lines towards IRAS $16293 \mathrm{~B}$ (in black) with the Gaussian fit in green dashed line, with fixed central velocity and FWHM. The same reference spectrum used in the analysis is represented in blue. The squared number in the top-right corner corresponds to the identification number of the transition, i.e. the panel number in Table D.1. 
Table D.1. $\mathrm{CHD}_{2} \mathrm{OH}$ identified lines.

\begin{tabular}{|c|c|c|c|c|}
\hline $\begin{array}{c}\text { Transition } \\
\left(J^{\prime \prime}, K^{\prime \prime}\right) \rightarrow\left(J^{\prime}, K^{\prime}\right)^{(b)}\end{array}$ & $\begin{array}{l}\text { Frequency } \\
\qquad(\mathrm{GHz})\end{array}$ & Panel number ${ }^{(a)}$ & $\begin{array}{c}W_{\text {IRAS } 16293 \mathrm{~A}^{(c)}} \\
\left(\mathrm{Jy} \mathrm{beam}^{-1} \mathrm{~km} \mathrm{~s}^{-1}\right)\end{array}$ & $\begin{array}{c}W_{\text {IRAS } 16293 \text { B }}{ }^{(c)} \\
\left(\mathrm{Jy} \mathrm{beam}^{-1} \mathrm{~km} \mathrm{~s}^{-1}\right)\end{array}$ \\
\hline$\left(7,1+\mathrm{e}_{1}\right) \rightarrow\left(8,1+\mathrm{e}_{1}\right)$ & 330.4602 & 0 & - & $0.362 \pm 0.013$ \\
\hline$\left(7,0 \mathrm{o}_{1}+\right) \rightarrow\left(8,0 \mathrm{o}_{1}+\right)$ & 330.6225 & 1 & - & - \\
\hline$\left(7,0 \mathrm{e}_{0}+\right) \rightarrow\left(8,0 \mathrm{e}_{0}+\right)$ & 331.7538 & 2 & - & - \\
\hline$\left(7,0 \mathrm{e}_{1}+\right) \rightarrow\left(8,0 \mathrm{e}_{1}+\right)$ & 332.2835 & 3 & $2.129 \pm 0.078$ & $0.314 \pm 0.014$ \\
\hline$\left(7,7 \pm \mathrm{e}_{1}\right) \rightarrow\left(8,7 \pm \mathrm{e}_{1}\right)$ & 332.3431 & 4 & $0.938 \pm 0.079$ & $0.239 \pm 0.014$ \\
\hline$\left(7,6 \pm \mathrm{e}_{1}\right) \rightarrow\left(8,6 \pm \mathrm{e}_{1}\right)$ & 332.4374 & 5 & - & $0.349 \pm 0.013$ \\
\hline$\left(7,2-\mathrm{e}_{1}\right) \rightarrow\left(8,2-\mathrm{e}_{1}\right)$ & 332.4448 & 6 & $2.139 \pm 0.094$ & $0.353 \pm 0.016$ \\
\hline$\left(7,5 \pm \mathrm{e}_{1}\right) \rightarrow\left(8,5 \pm \mathrm{e}_{1}\right)$ & 332.5087 & 7 & - & $0.353 \pm 0.015$ \\
\hline$\left(7,4 \pm \mathrm{e}_{1}\right) \rightarrow\left(8,4 \pm \mathrm{e}_{1}\right)$ & 332.5498 & 8 & $2.382 \pm 0.081$ & $0.370 \pm 0.014$ \\
\hline$\left(7,2+\mathrm{e}_{1}\right) \rightarrow\left(8,2+\mathrm{e}_{1}\right)$ & 332.7186 & 9 & - & - \\
\hline$\left(7,7 \mathrm{e}_{0} \pm\right) \rightarrow\left(8,7 \mathrm{e}_{0} \pm\right)$ & 333.0702 & 10 & - & $0.286 \pm 0.014$ \\
\hline$\left(7,2-\mathrm{o}_{1}\right) \rightarrow\left(8,2-\mathrm{o}_{1}\right)$ & 333.0730 & 11 & - & $0.391 \pm 0.016$ \\
\hline$\left(7,5 \pm o_{1}\right) \rightarrow\left(8,5 \pm o_{1}\right)$ & 333.1202 & 12 & $2.290 \pm 0.107$ & $0.376 \pm 0.015$ \\
\hline$\left(7,6 \pm o_{1}\right) \rightarrow\left(8,6 \pm o_{1}\right)$ & 333.1347 & 13 & $1.195 \pm 0.081$ & $0.350 \pm 0.016$ \\
\hline$\left(7,6 \mathrm{e}_{0} \pm\right) \rightarrow\left(8,6 \mathrm{e}_{0} \pm\right)$ & 333.1595 & 14 & - & $0.254 \pm 0.015$ \\
\hline$\left(7,7 \pm o_{1}\right) \rightarrow\left(8,7 \pm o_{1}\right)$ & 333.1639 & 15 & $2.403 \pm 0.091$ & $0.442 \pm 0.016$ \\
\hline$\left(7,5 \mathrm{e}_{0} \pm\right) \rightarrow\left(8,5 \mathrm{e}_{0} \pm\right)$ & 333.2504 & 16 & $1.645 \pm 0.082$ & $0.396 \pm 0.015$ \\
\hline$\left(7,3+\mathrm{e}_{1}\right) \rightarrow\left(8,3+\mathrm{e}_{1}\right)$ & 333.3351 & & $1.579+0.054$ & $0.371+0.013$ \\
\hline$\left(7,3+o_{1}\right) \rightarrow\left(8,3+o_{1}\right)$ & 333.3361 & 17 & $1.519 \pm 0.034$ & $0.5 / 1 \pm 0.015$ \\
\hline$\left(7,3-o_{1}\right) \rightarrow\left(8,3-o_{1}\right)$ & 333.3502 & & & \\
\hline$\left(7,4 \mathrm{e}_{0} \pm\right) \rightarrow\left(8,4 \mathrm{e}_{0} \pm\right)$ & 333.3502 & 18 & - & $0.467 \pm 0.013$ \\
\hline$\left(7,3-\mathrm{e}_{1}\right) \rightarrow\left(8,3-\mathrm{e}_{1}\right)$ & 333.3509 & & & \\
\hline$\left(7,2-\mathrm{e}_{0}\right) \rightarrow\left(8,2-\mathrm{e}_{0}\right)$ & 334.0098 & 19 & $1.904 \pm 0.080$ & $0.389 \pm 0.014$ \\
\hline$\left(7,2+\mathrm{e}_{0}\right) \rightarrow\left(8,2+\mathrm{e}_{0}\right)$ & 334.0568 & 20 & $1.849 \pm 0.074$ & $0.362 \pm 0.012$ \\
\hline$\left(7,1-\mathrm{e}_{1}\right) \rightarrow\left(8,1-\mathrm{e}_{1}\right)$ & 334.4162 & 21 & $1.929 \pm 0.069$ & $0.407 \pm 0.013$ \\
\hline$\left(7,2+o_{1}\right) \rightarrow\left(8,2+o_{1}\right)$ & 334.4503 & 22 & $1.856 \pm 0.079$ & $0.429 \pm 0.015$ \\
\hline$\left(4,3 \mathrm{o}_{1}\right) \rightarrow\left(4,4 \mathrm{e}_{0}\right)$ & 336.3732 & 23 & - & - \\
\hline$\left(5,3 \mathrm{o}_{1}\right) \rightarrow\left(5,4 \mathrm{e}_{0}\right)$ & 336.3804 & 24 & - & - \\
\hline$\left(6,3 \mathrm{o}_{1}\right) \rightarrow\left(6,4 \mathrm{e}_{0}\right)$ & 336.3948 & 25 & $0.107 \pm 0.207$ & $0.049 \pm 0.013$ \\
\hline$\left(7,3 \mathrm{o}_{1}\right) \rightarrow\left(7,4 \mathrm{e}_{0}\right)$ & 336.4197 & 26 & - & $0.341 \pm 0.013$ \\
\hline$\left(8,3 \mathrm{o}_{1}\right) \rightarrow\left(8,4 \mathrm{e}_{0}\right)$ & 336.4520 & 27 & $1.806 \pm 0.078$ & $0.367 \pm 0.014$ \\
\hline$\left(7,1-\mathrm{e}_{0}\right) \rightarrow\left(8,1-\mathrm{e}_{0}\right)$ & 336.9568 & 28 & $2.042 \pm 0.072$ & $0.414 \pm 0.013$ \\
\hline$\left(7,1-o_{1}\right) \rightarrow\left(8,1-o_{1}\right)$ & 337.7278 & 29 & - & - \\
\hline$\left(6,5 \mathrm{e}_{1}\right) \rightarrow\left(7,6 \mathrm{e}_{1}\right)$ & 353.9833 & 30 & - & - \\
\hline
\end{tabular}

Notes. The quantum numbers are taken from Mukhopadhyay (2016). ${ }^{(a)}$ This corresponds to the number indicated in the top-right corner of each panel in Figs. D.1 and D.2. ${ }^{(b)} \mathrm{e}_{0}, \mathrm{o}_{1}$ and $\mathrm{e}_{1}$ corresponds to the three first torsional states. The notation of the quantum numbers is directly taken from Mukhopadhyay (2016). ${ }^{(c)} W$ is the integrated intensity of the unblended lines, summed over the range $\left[v_{0}-\Delta v_{\mathrm{FWHM}}, v_{0}+\Delta v_{\mathrm{FWHM}}\right]$, given $\Delta v_{\mathrm{FWHM}}=v_{0} \frac{F W H M}{\mathrm{c}}$, with FWHM equal to 2.2 and $0.8 \mathrm{~km} \mathrm{~s}^{-1}$ for IRAS $16293 \mathrm{~A}$ and B, respectively. 


\section{Appendix E: Synthetic spectra}

Figures E.1-E.10 show the synthetic spectra produced by the LTE model using the parameters listed in Tables 1 and 2 , for all the detected species. The most unblended and optically thin lines are shown, up to twenty lines for the species having more fitted transitions, such as $\mathrm{CH}_{3} \mathrm{OCHO}$ and $\mathrm{CH}_{3} \mathrm{COCH}_{3}$.
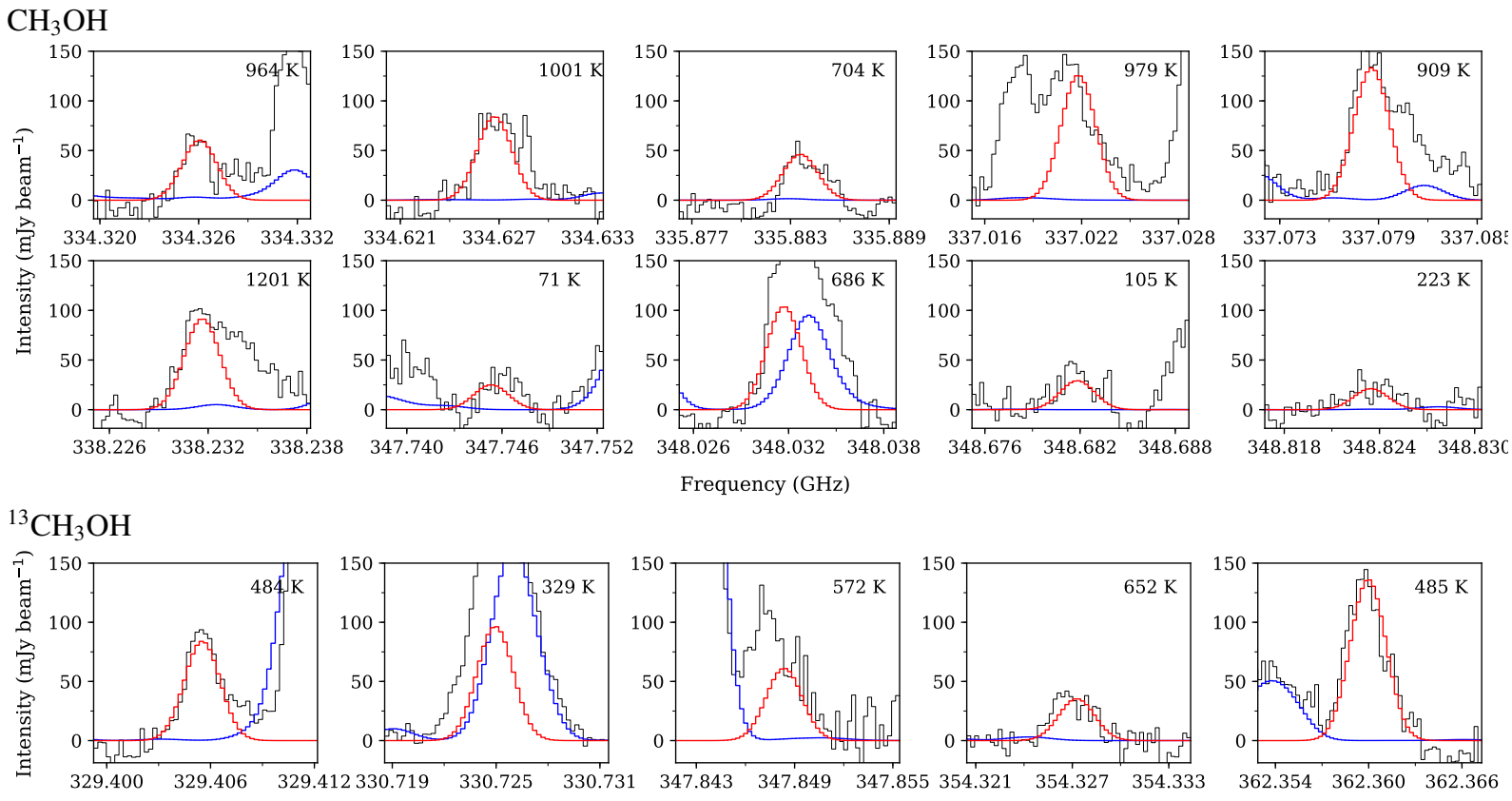

$\mathrm{CH}_{3}^{18} \mathrm{OH}$
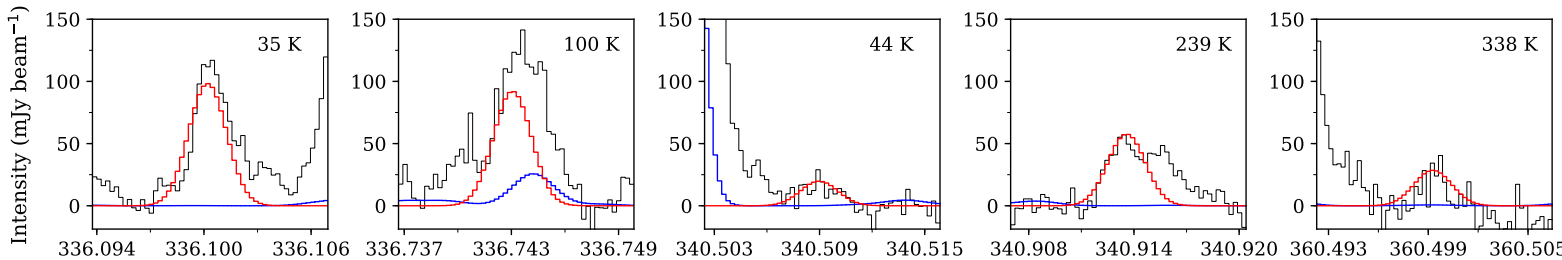

Frequency $(\mathrm{GHz})$

\section{$\mathrm{CH}_{2} \mathrm{DOH}$}
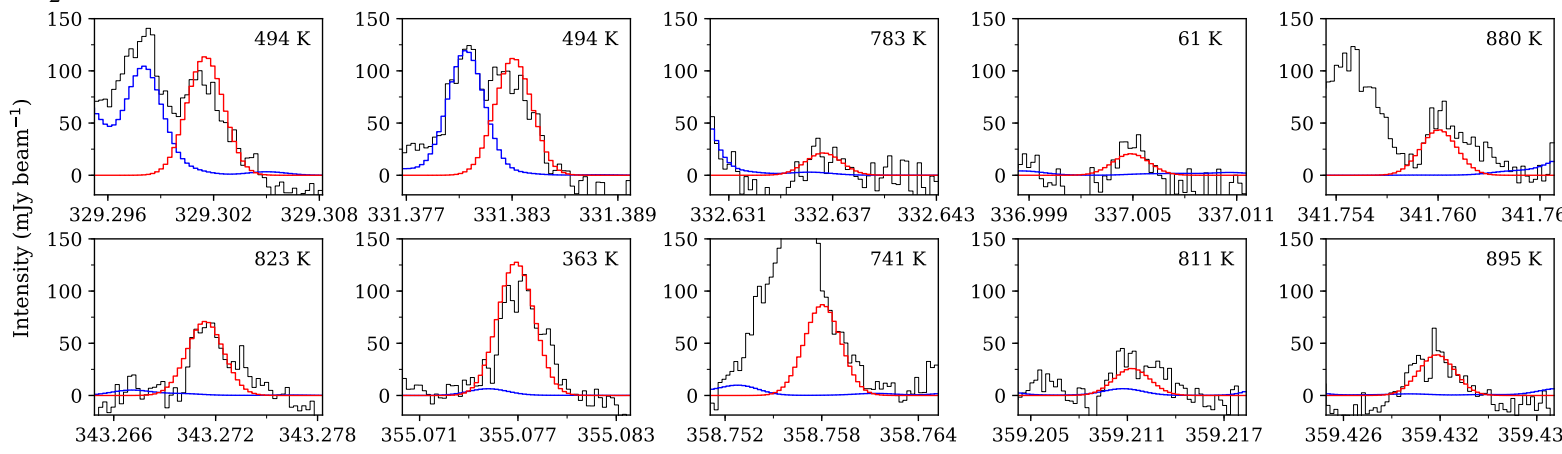

$\mathrm{CH}_{3} \mathrm{OD}$
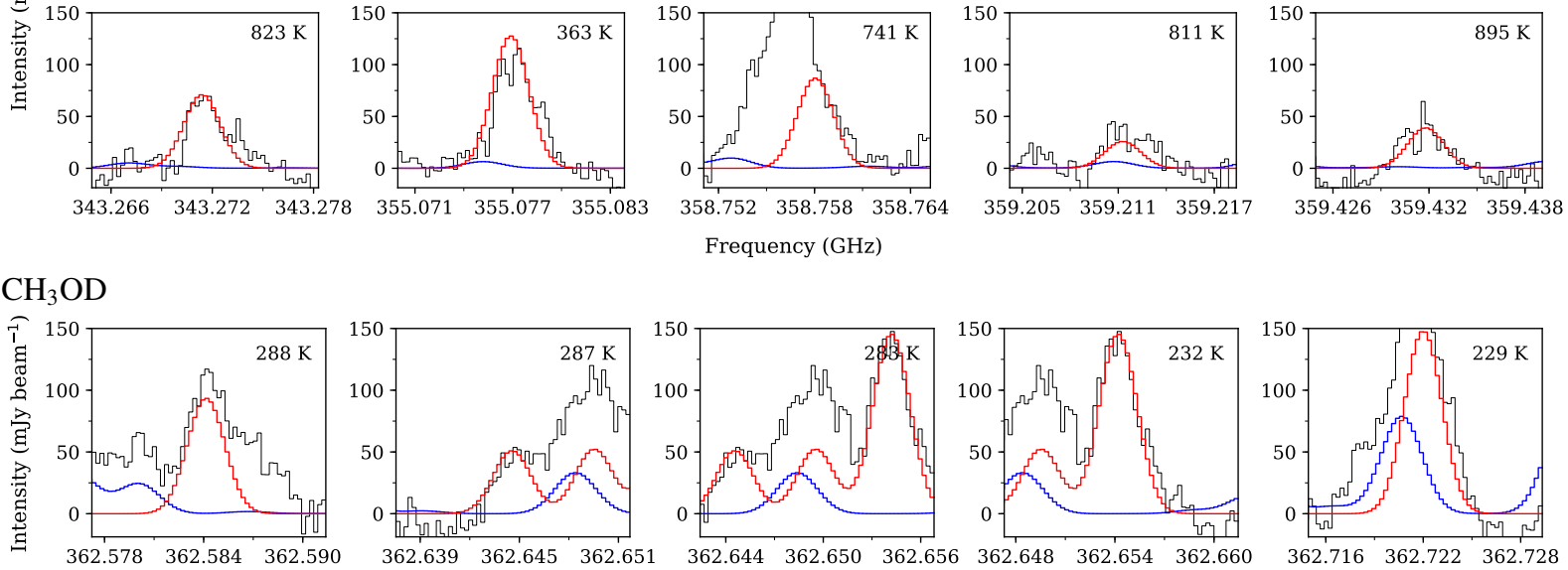

Frequency $(\mathrm{GHz})$

$$
\text { Frequency (GHz) }
$$

Fig. E.1. Representative selection of transitions of $\mathrm{CH}_{3} \mathrm{OH}$ isotopologues towards IRAS 16293 A. The synthetic spectra is over-plotted in red, the reference spectrum in blue, and the data in black. The upper energy level of the transition is indicated in the top-left corner. 
$\mathrm{CH}_{3} \mathrm{OCH}_{3}$
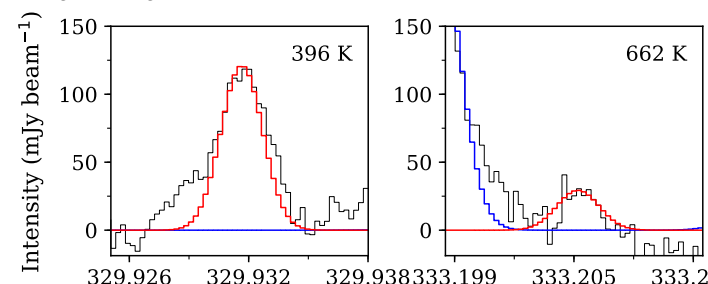

$\begin{array}{lllll}329.926 & 329.932 & 329.938333 .199 & 333.205 & 333.21\end{array}$

\section{$\mathrm{CH}_{3} \mathrm{O}^{13} \mathrm{CH}_{3}$}
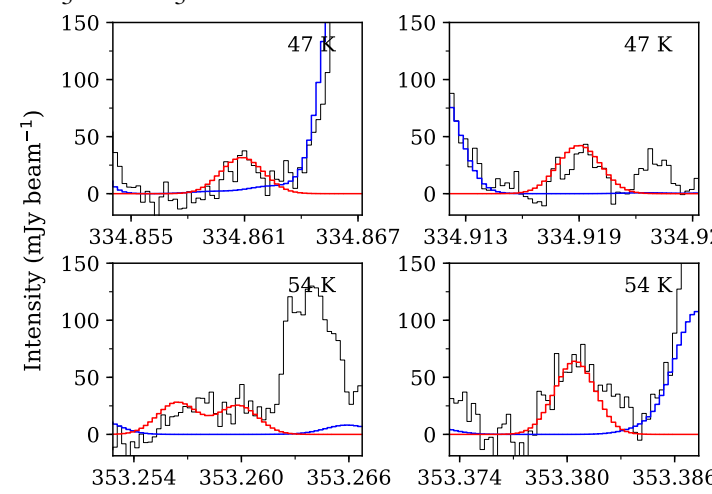

\section{a- $\mathrm{CH}_{2} \mathrm{DOCH}_{3}$}

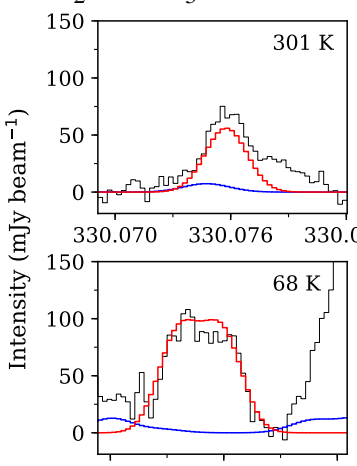

$\begin{array}{lll}335.465 & 335.471 & 335.477\end{array}$
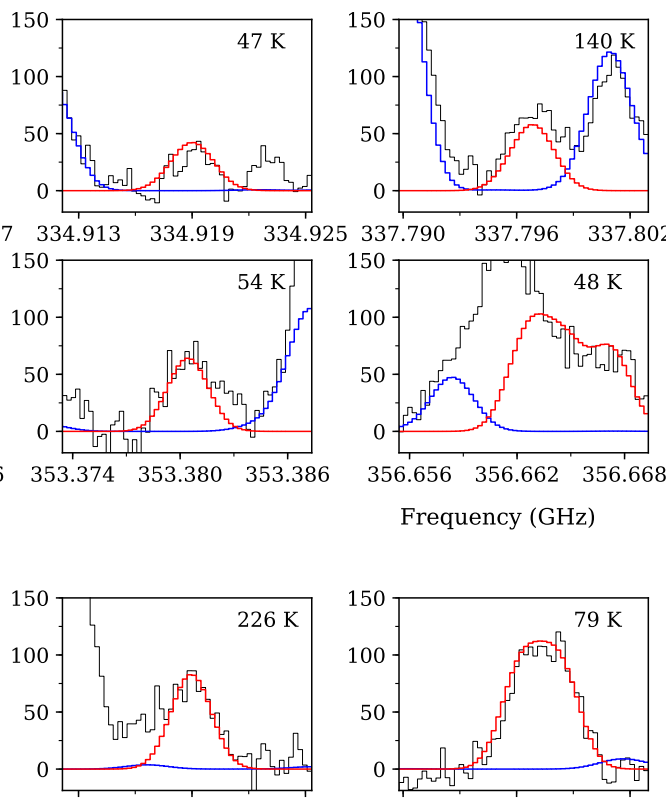

$\begin{array}{lll}356.656 & 356.662 & 356.668\end{array}$ Frequency (GHz)
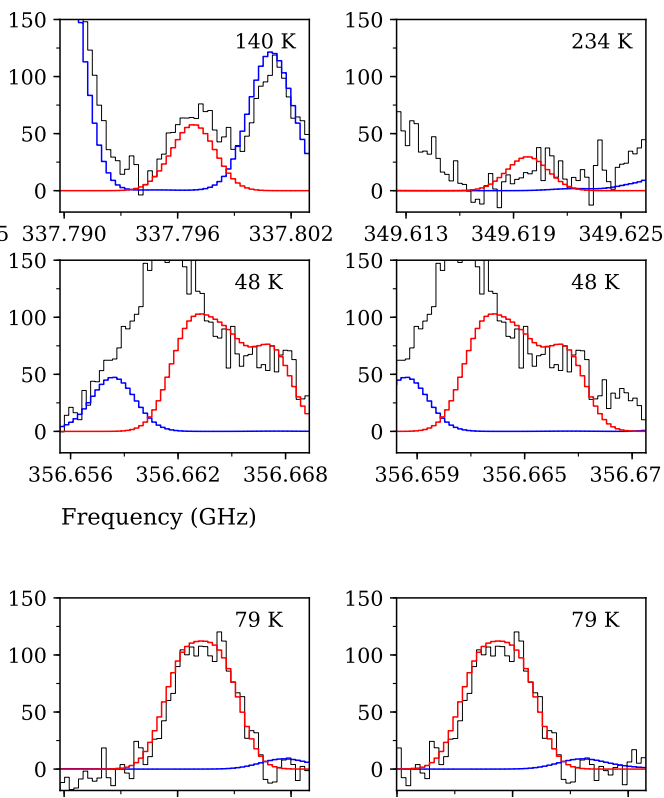

$\begin{array}{lll}356.659 & 356.665 & 356.67\end{array}$

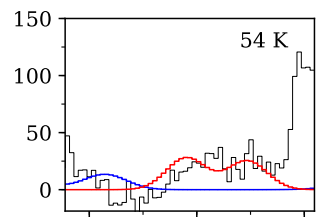

$\begin{array}{lll}353.251 & 353.257 & 353.263\end{array}$

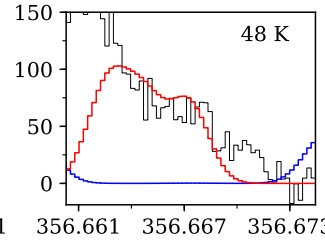

$\begin{array}{lll}356.661 & 356.667 & 356.673\end{array}$
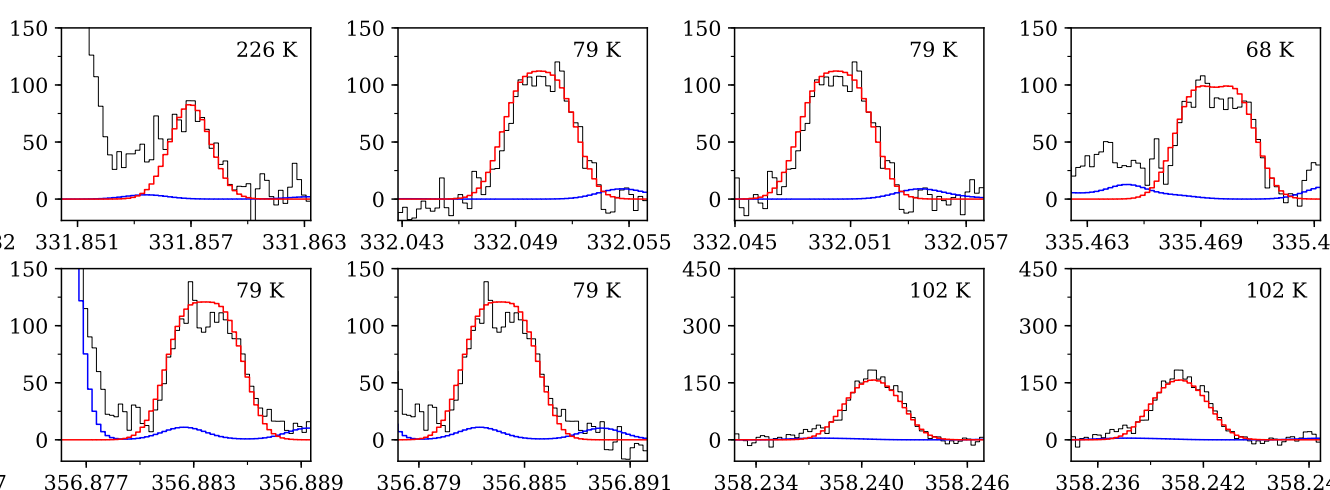

$\begin{array}{lll}335.463 & 335.469 & 335.475\end{array}$

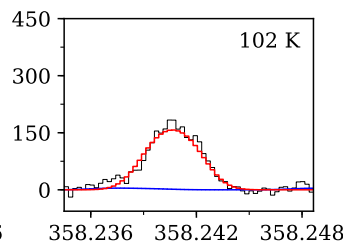

Frequency $(\mathrm{GHz})$

\section{$\mathrm{s}-\mathrm{CH}_{2} \mathrm{DOCH}_{3}$}
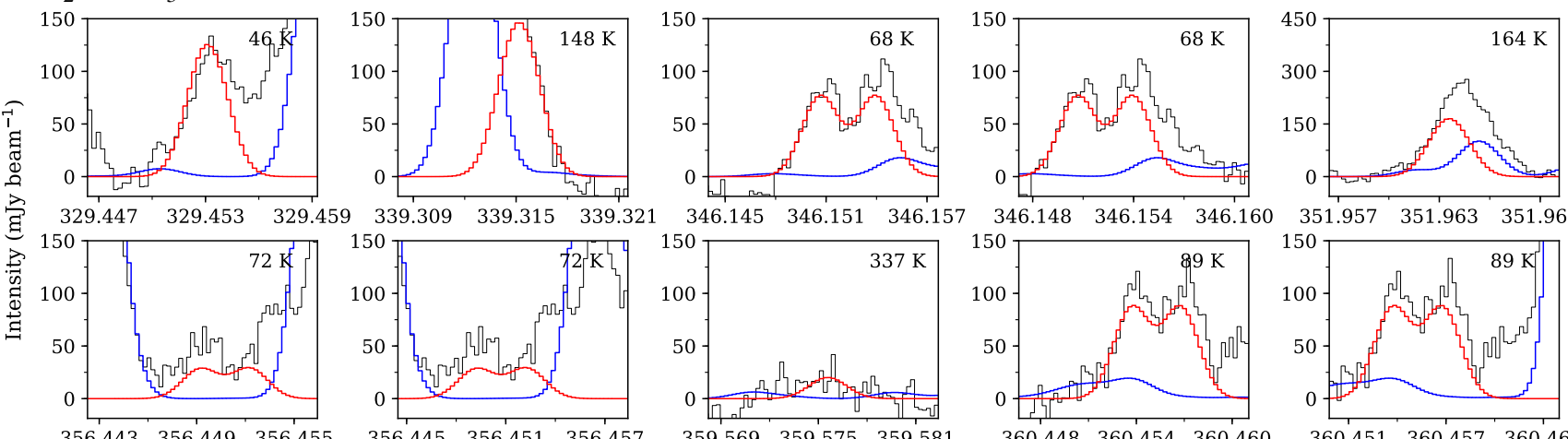

$\begin{array}{lll}356.445 & 356.451 & 356.457\end{array}$

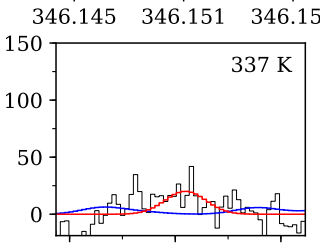

$359.569 \quad 359.575 \quad 359.581$ Frequency $(\mathrm{GHz})$

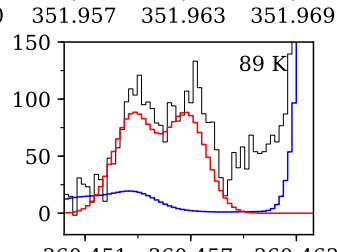

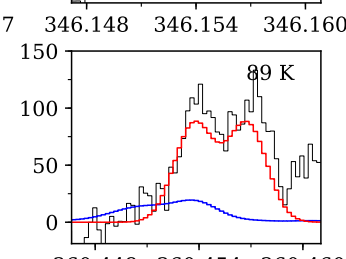

$\begin{array}{lll}360.451 & 360.457 & 360.463\end{array}$

Fig. E.2. Representatives selection of transitions of $\mathrm{CH}_{3} \mathrm{OCH}_{3}$ isotopologues towards IRAS $16293 \mathrm{~A}$. The synthetic spectra is over-plotted in red, the reference spectrum in blue, and the data in black. The upper energy level of the transition is indicated in the top-left corner. 


\section{$\mathrm{H}_{2} \mathrm{CO}$}
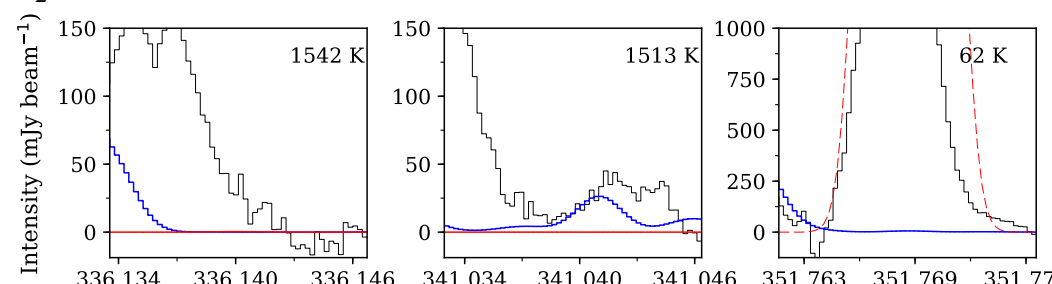

$\begin{array}{lllllll}341.034 & 341.040 & 341.046 & 351.763 & 351.769 & 351.775\end{array}$ Frequency $(\mathrm{GHz})$

\section{$\mathrm{H}_{2}^{13} \mathrm{CO}$}
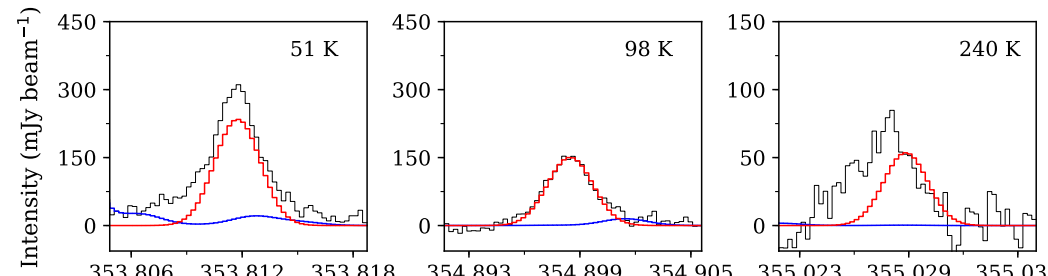

$\begin{array}{lllllll}354.893 & 354.899 & 354.905 & 355.023 & 355.029 & 355.035\end{array}$

Frequency $(\mathrm{GHz})$

\section{$\mathrm{H}_{2} \mathrm{C}^{18} \mathrm{O}$}
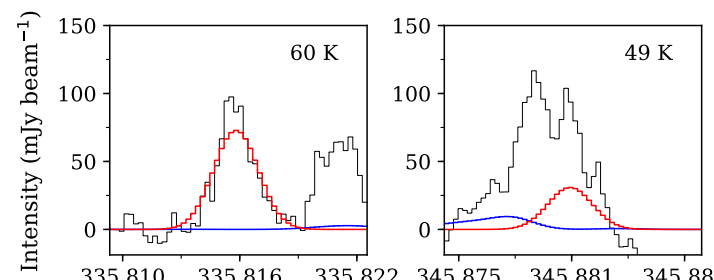

$\begin{array}{llllll}335.810 & 335.816 & 335.822 & 345.875 & 345.881 & 345.887\end{array}$
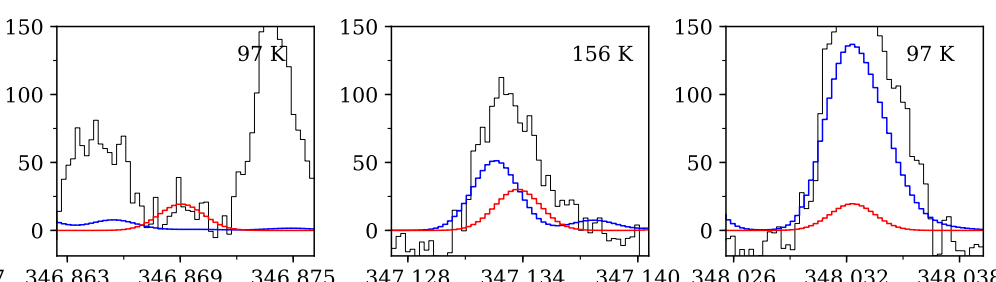

Frequency (GHz)

\section{$\mathrm{HDCO}$}
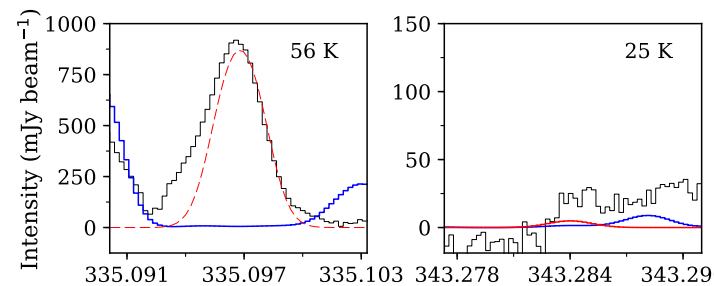

\section{$\mathrm{D}_{2} \mathrm{CO}$}

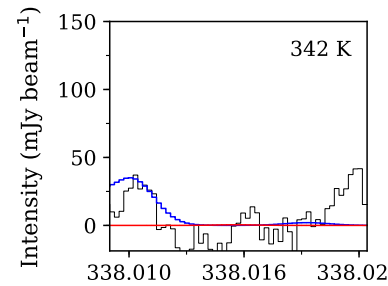

$\begin{array}{llllll}338.010 & 338.016 & 338.022 & 345.069 & 345.075 & 345.081\end{array}$
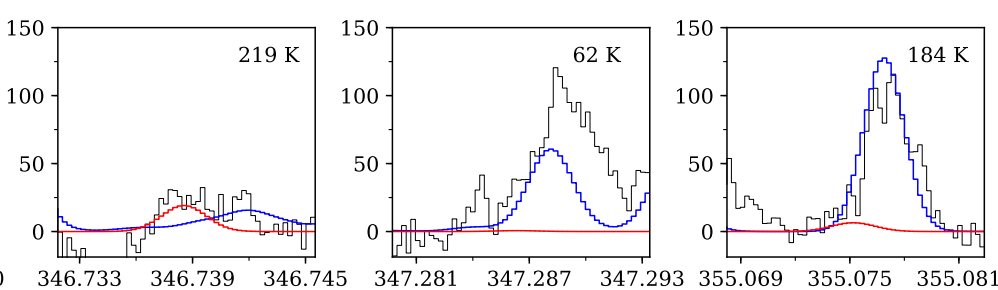

Frequency (GHz)

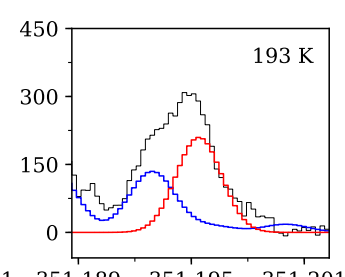

Frequency (GHz)

$\begin{array}{llllll}347.281 & 347.287 & 347.293 & 355.069 & 355.075 & 355.081\end{array}$
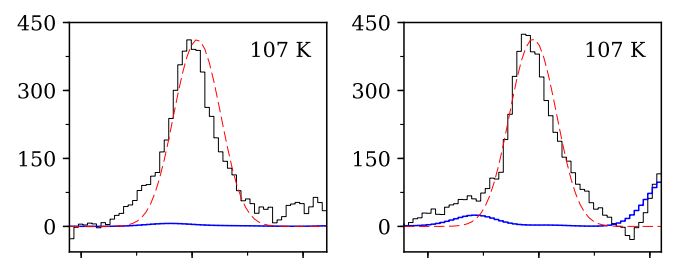

$351.888 \quad 351.894 \quad 351.900$

$\begin{array}{lll}352.238 & 352.244 & 352.250\end{array}$
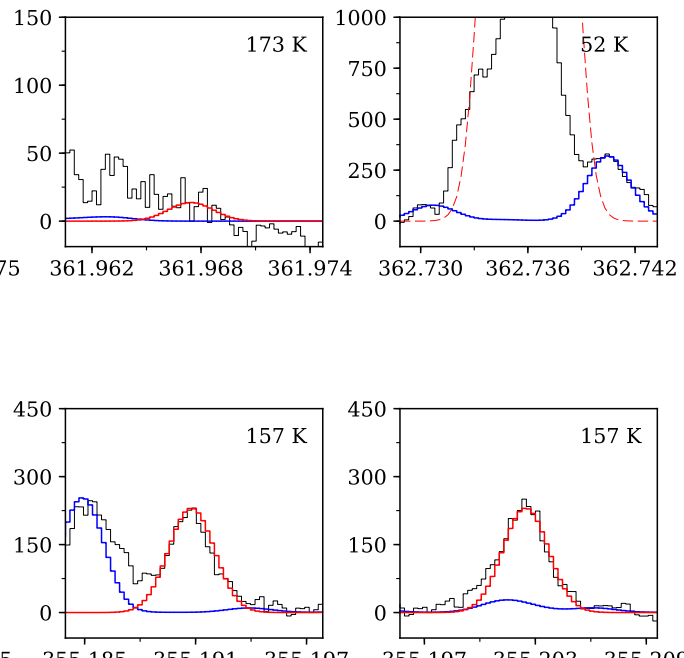

$\begin{array}{lll}355.197 & 355.203 & 355.209\end{array}$

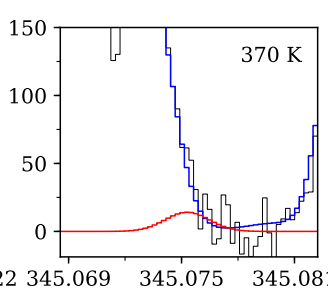

Fig. E.3. Representative selection of transitions of $\mathrm{H}_{2} \mathrm{CO}$ isotopologues towards IRAS 16293 A. The synthetic spectra is over-plotted in red, the reference spectrum in blue, and the data in black. The dashed curves represent optically thick lines. The upper energy level of the transition is indicated in the top-left corner. 


\section{$\mathrm{C}_{2} \mathrm{H}_{5} \mathrm{OH}$}
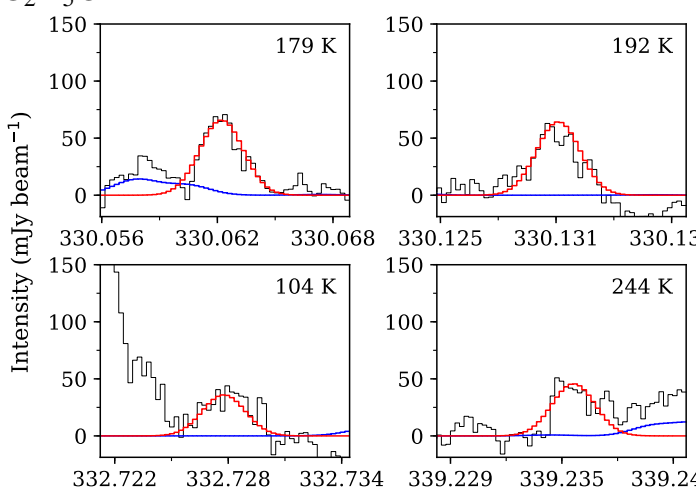

$\begin{array}{llllll}332.722 & 332.728 & 332.734 & 339.229 & 339.235 & 339.241\end{array}$

\section{$a-\mathrm{CH}_{3} \mathrm{CHDOH}$}
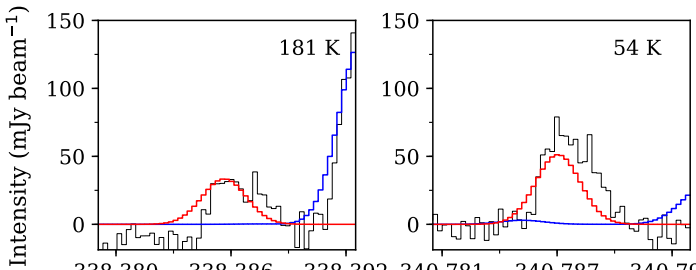

$\begin{array}{llllll}338.380 & 338.386 & 338.392 & 340.781 & 340.787 & 340.793\end{array}$

\section{a- $\mathrm{CH}_{3} \mathrm{CH}_{2} \mathrm{OD}$}
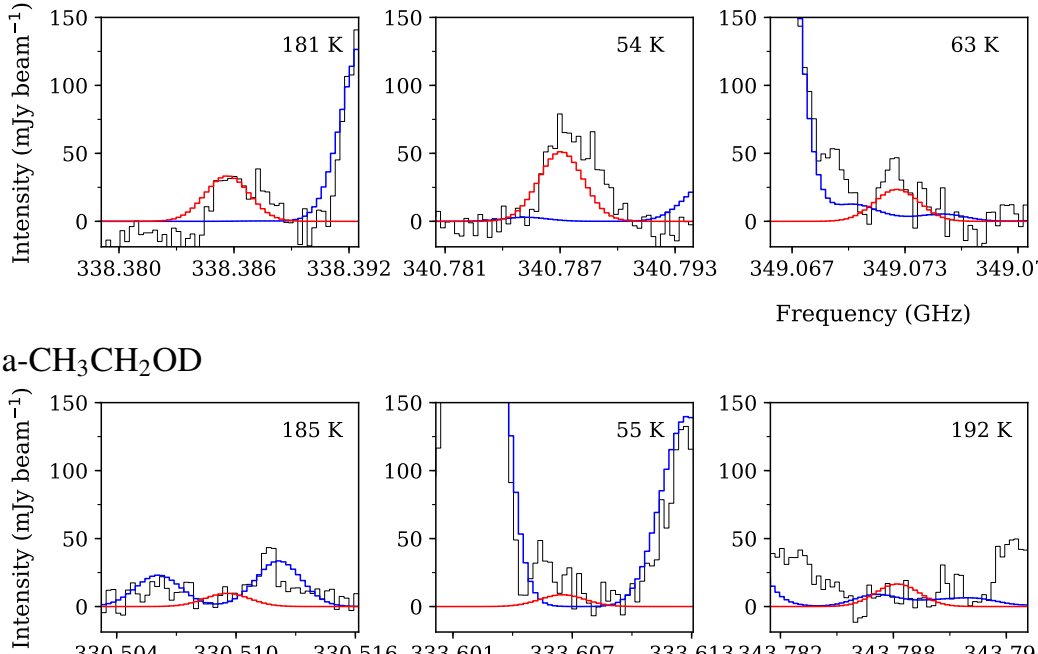

$\begin{array}{llllll}349.067 & 349.073 & 349.079 & 354.098 & 354.104 & 354.110\end{array}$ Frequency $(\mathrm{GHz})$

$\begin{array}{llllllll}330.504 & 330.510 & 330.516 & 333.601 & 333.607 & 333.613343 .782 & 343.788 & 343.794\end{array}$
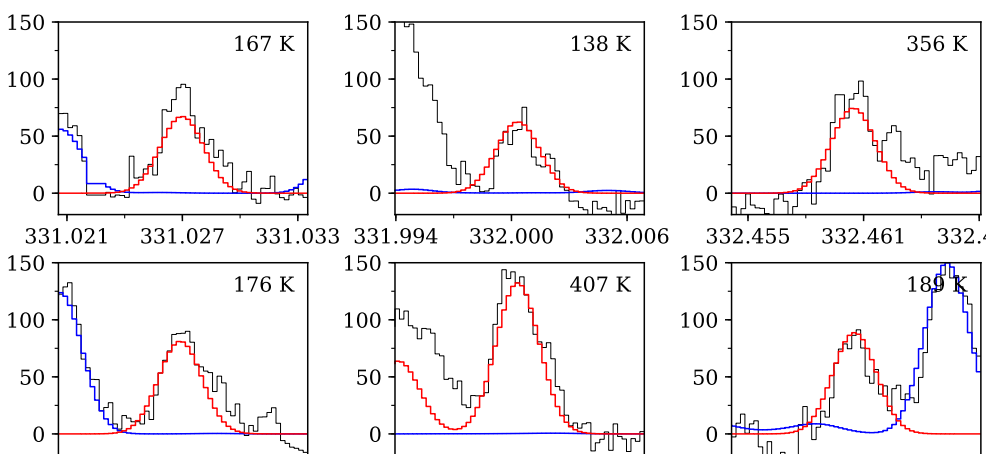

$\begin{array}{llllll}339.277 & 339.283 & 339.289 & 346.356 & 346.362 & 346.368\end{array}$ Frequency $(\mathrm{GHz})$

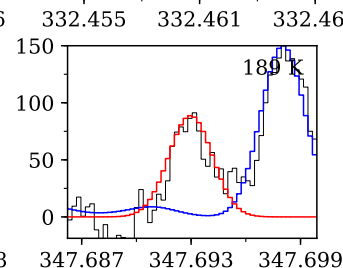
Frequency $(\mathrm{GHz})$

\section{$\mathrm{a}-\mathrm{a}-\mathrm{CH}_{2} \mathrm{DCH}_{2} \mathrm{OH}$}

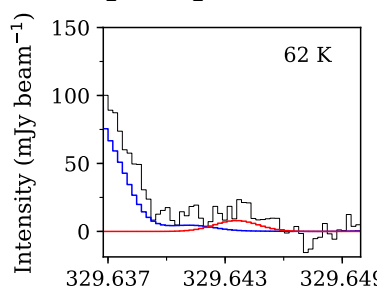

$\begin{array}{llllll}329.637 & 329.643 & 329.649 & 333.214 & 333.220 & 333.2\end{array}$

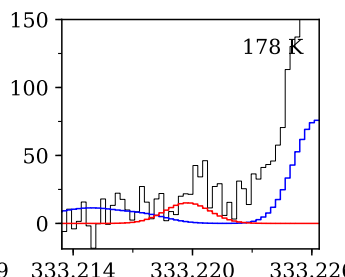

a-s- $\mathrm{CH}_{2} \mathrm{DCH}_{2} \mathrm{OH}$
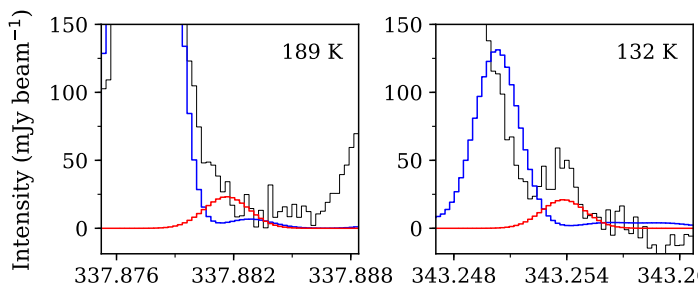

\begin{tabular}{lll}
\hline 1 & & \\
\hline 43.248 & 343.254 & 343.260
\end{tabular}
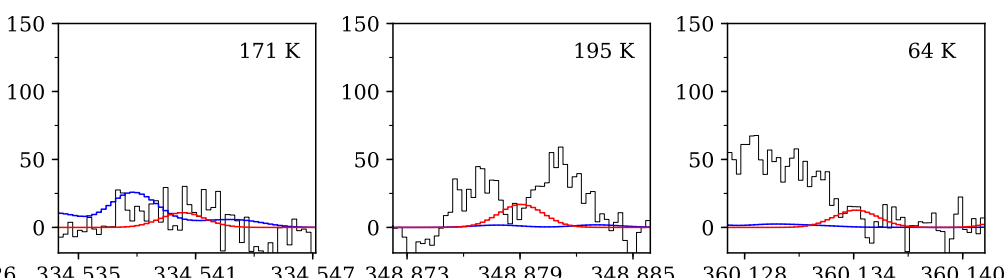

Frequency $(\mathrm{GHz})$
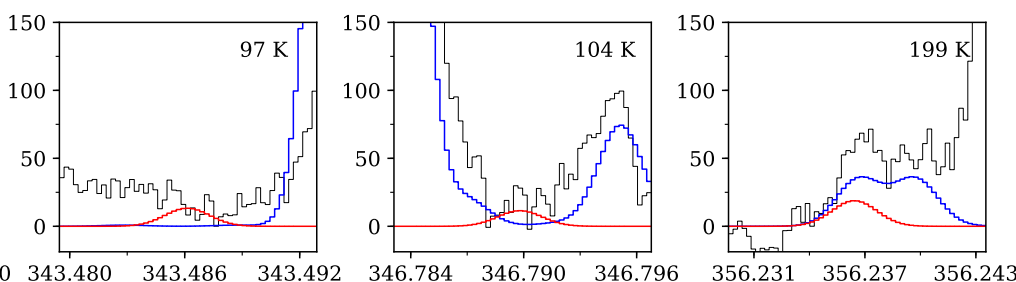

Frequency $(\mathrm{GHz})$

$\begin{array}{lll}360.128 & 360.134 & 360.140\end{array}$

$\begin{array}{lll}346.784 & 346.790 & 346.79\end{array}$
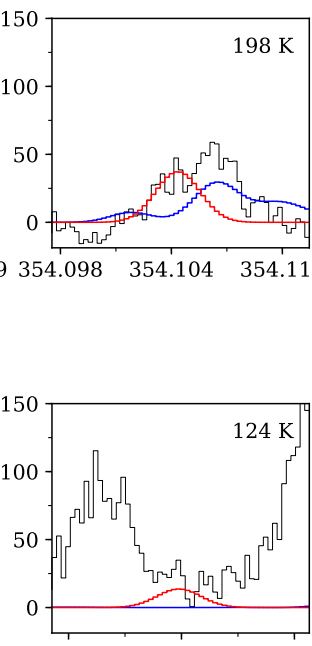

$\begin{array}{lllllll}344.567 & 344.573 & 344.579 & 354.042 & 354.048 & 354.054\end{array}$

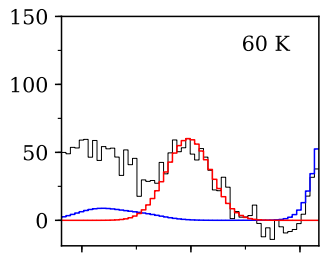

$357.969 \quad 357.975 \quad 357.981$

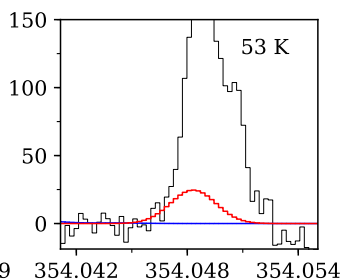

Fig. E.4. Representative selection of transitions of $\mathrm{C}_{2} \mathrm{H}_{5} \mathrm{OH}$ isotopologues towards IRAS 16293 A. The synthetic spectra is over-plotted in red, the reference spectrum in blue, and the data in black. The upper energy level of the transition is indicated in the top-left corner. 
S. Manigand et al.: Inventory of complex organic molecules towards IRAS 16293-2422 A

\section{$\mathrm{t}-\mathrm{HCOOH}$}
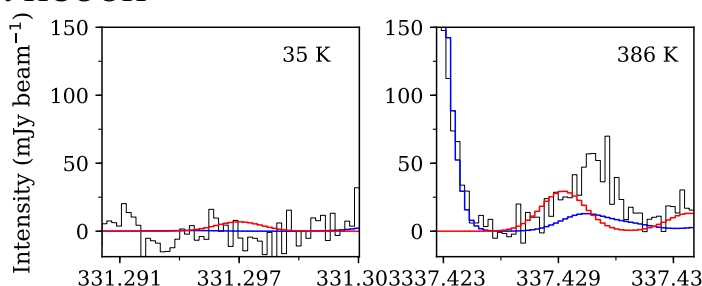

\section{$\mathrm{t}-\mathrm{H}^{13} \mathrm{COOH}$}
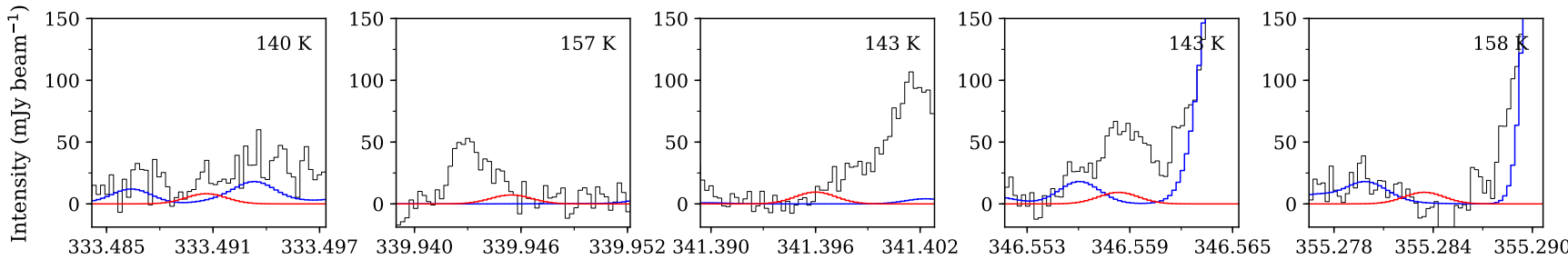

Frequency $(\mathrm{GHz})$

\section{$\mathrm{t}-\mathrm{DCOOH}$}
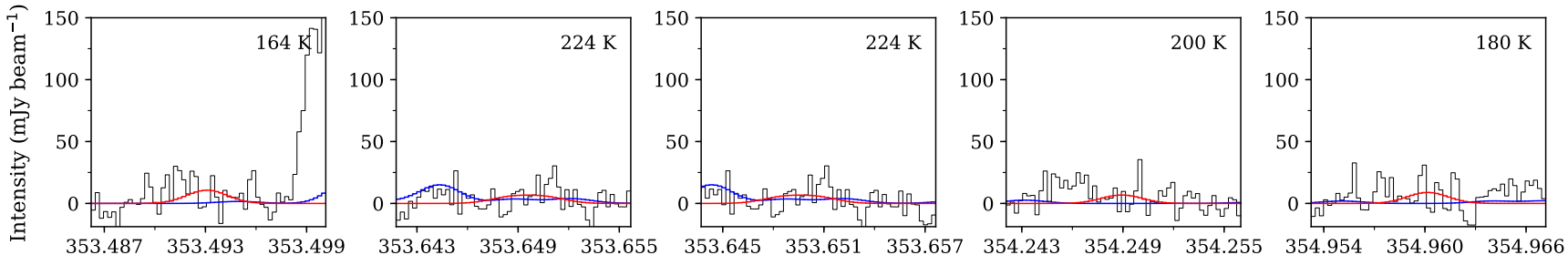

Frequency $(\mathrm{GHz})$

\section{$\mathrm{t}-\mathrm{HCOOD}$}
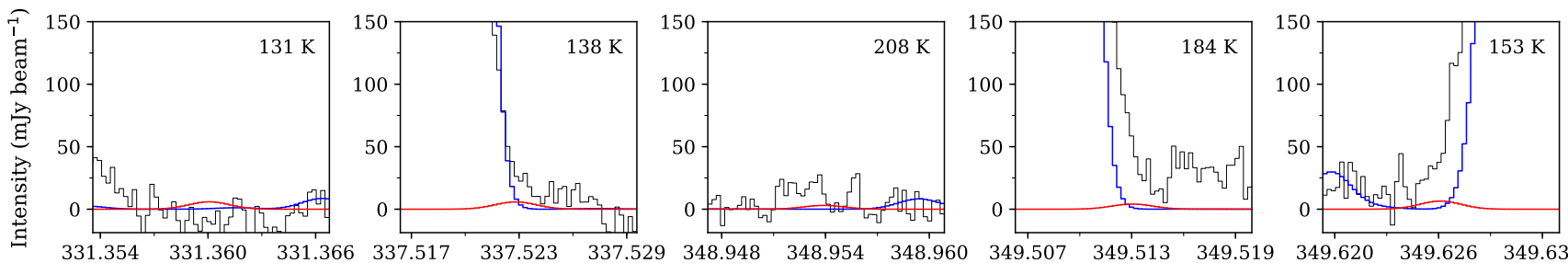

Frequency (GHz)

\section{$\mathrm{CH}_{3} \mathrm{COOH}$}
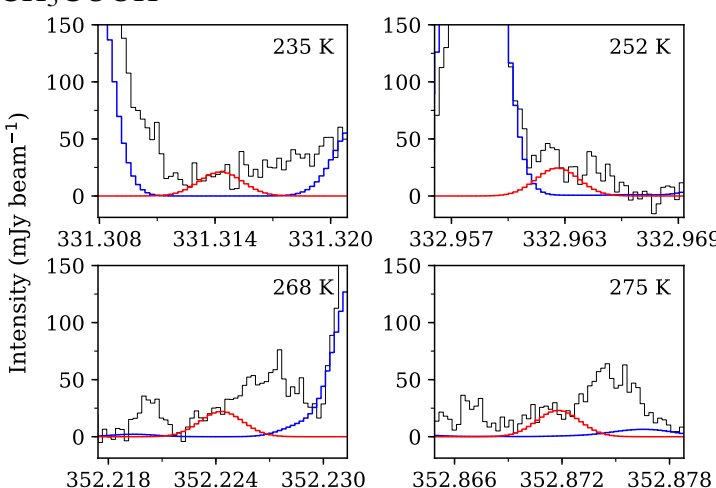
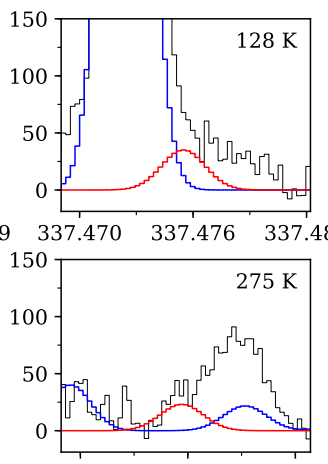

$353.156 \quad 353.162 \quad 353.168$ Frequency $(\mathrm{GHz})$
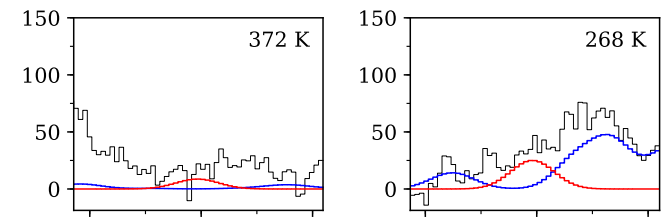

$\begin{array}{lllllll}340.058 & 340.064 & 340.070 & 343.825 & 343.831 & 343.837\end{array}$
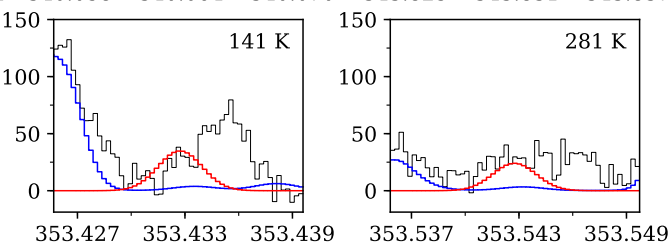

Fig. E.5. Representative selection of transitions of t-HCOOH isotopologues and $\mathrm{CH}_{3} \mathrm{COOH}$ towards IRAS 16293 A. The synthetic spectra is over-plotted in red, the reference spectrum in blue, and the data in black. The upper energy level of the transition is indicated in the top-left corner. 


\section{$\mathrm{CH}_{2} \mathrm{CO}$}
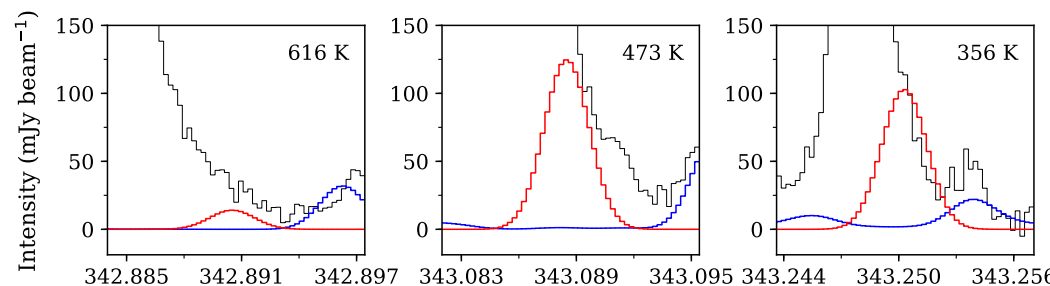

$\begin{array}{lllllllll}342.885 & 342.891 & 342.897 & 343.083 & 343.089 & 343.095 & 343.244 & 343.250 & 343.256\end{array}$

Frequency (GHz)

\section{CHDCO}
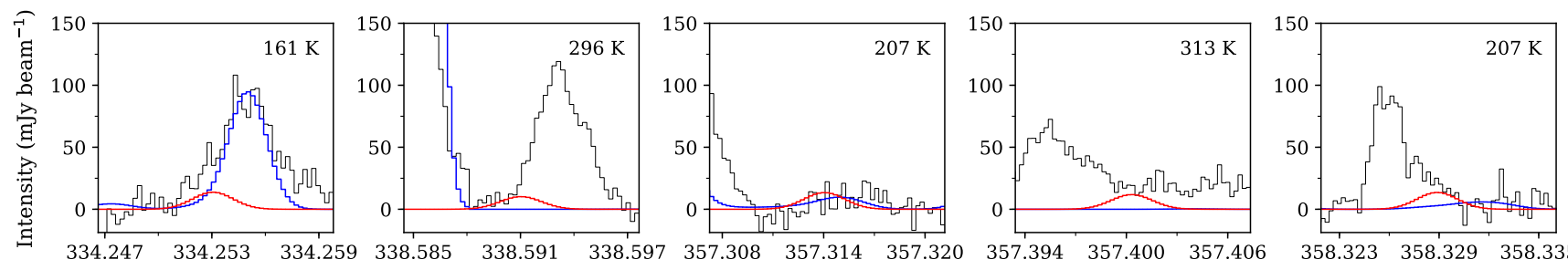

Frequency (GHz)

\section{$\mathrm{HNCO}$}
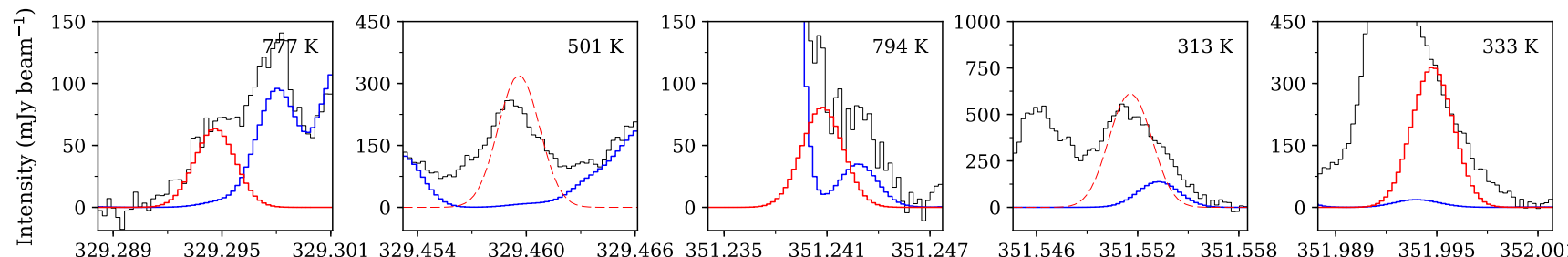

Frequency $(\mathrm{GHz})$

\section{DNCO}
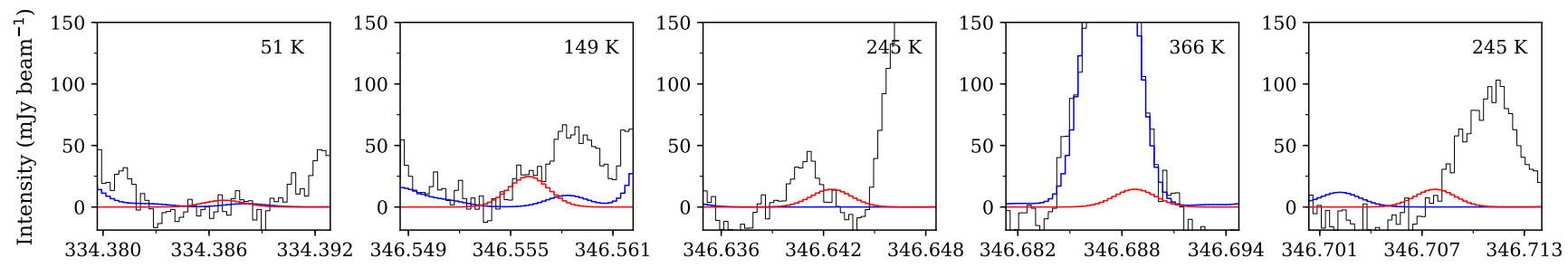

Frequency (GHz)

\section{$\mathrm{CH}_{3} \mathrm{CHO}$}
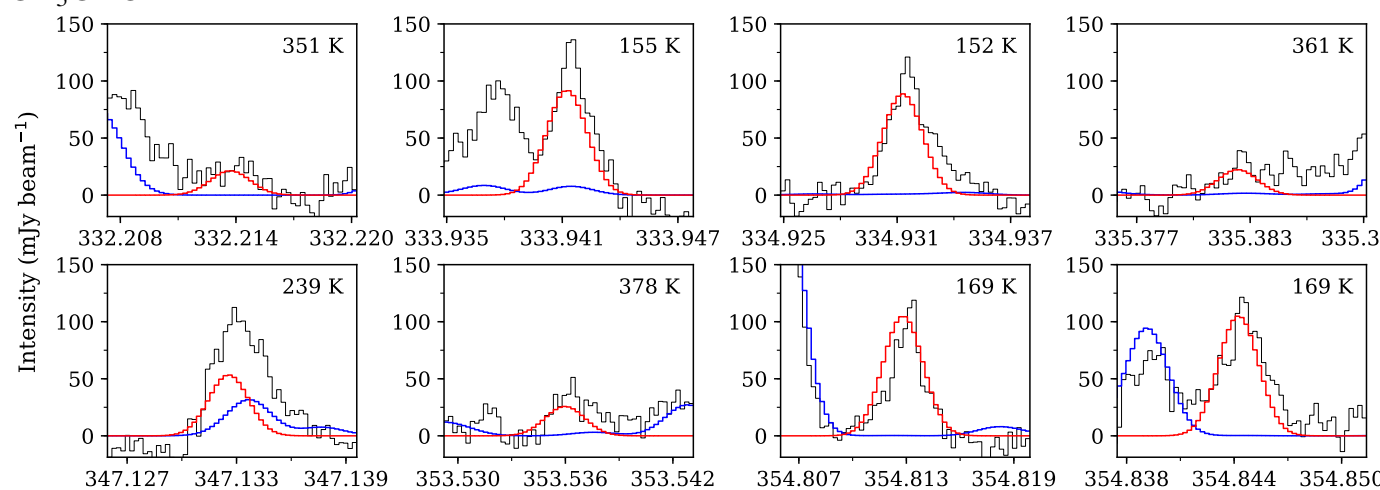

$335.377 \quad 335.383 \quad 335.38$

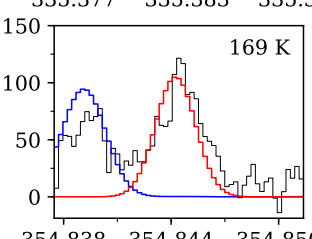

$\begin{array}{lllllll}354.807 & 354.813 & 354.819 & 354.838 & 354.844 & 354.850\end{array}$

Frequency $(\mathrm{GHz})$
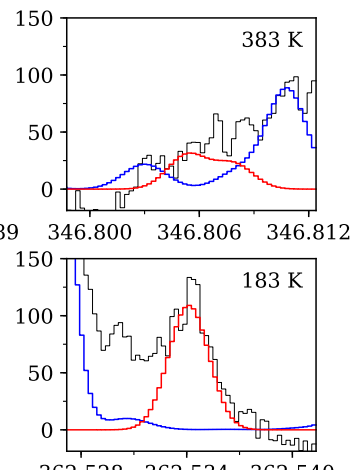

$\begin{array}{lll}362.528 & 362.534 & 362.540\end{array}$

Fig. E.6. Representative selection of transitions of $\mathrm{CH}_{2} \mathrm{CO}$ and $\mathrm{HNCO}$ isotopologues and $\mathrm{CH}_{3} \mathrm{CHO}$ towards IRAS 16293 A. The synthetic spectra is over-plotted in red, the reference spectrum in blue, and the data in black. The upper energy level of the transition is indicated in the top-left corner. 


\section{$\mathrm{CH}_{3} \mathrm{COCH}_{3}$}
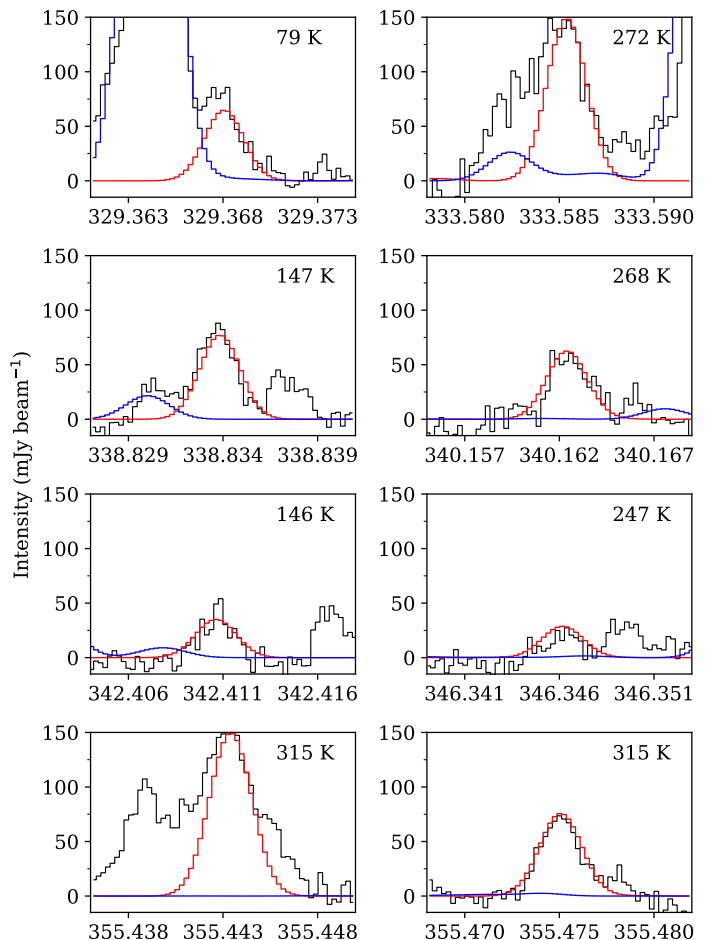

\section{$\mathrm{c}-\mathrm{H}_{2} \mathrm{COCH}_{2}$}
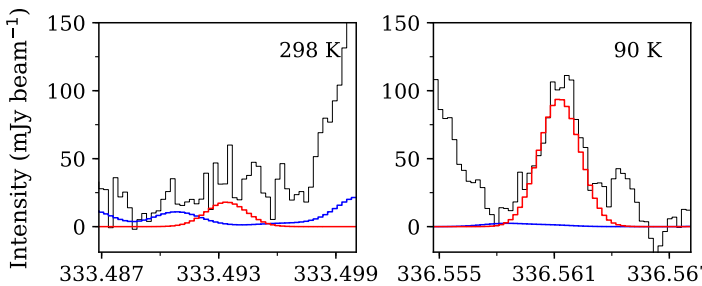

\section{$\mathrm{CH}_{3} \mathrm{O}^{13} \mathrm{CHO}$}
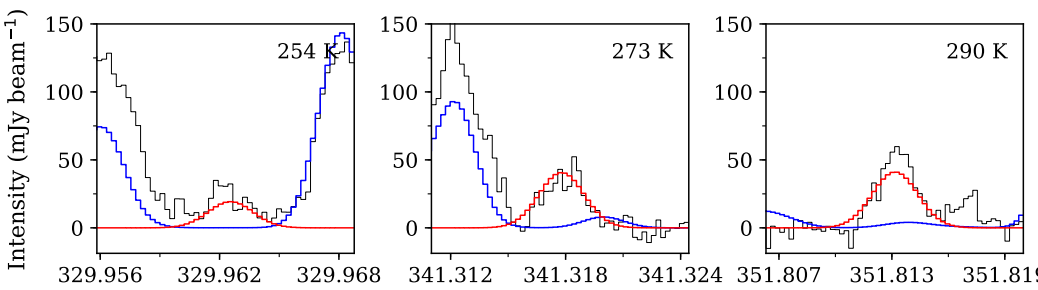
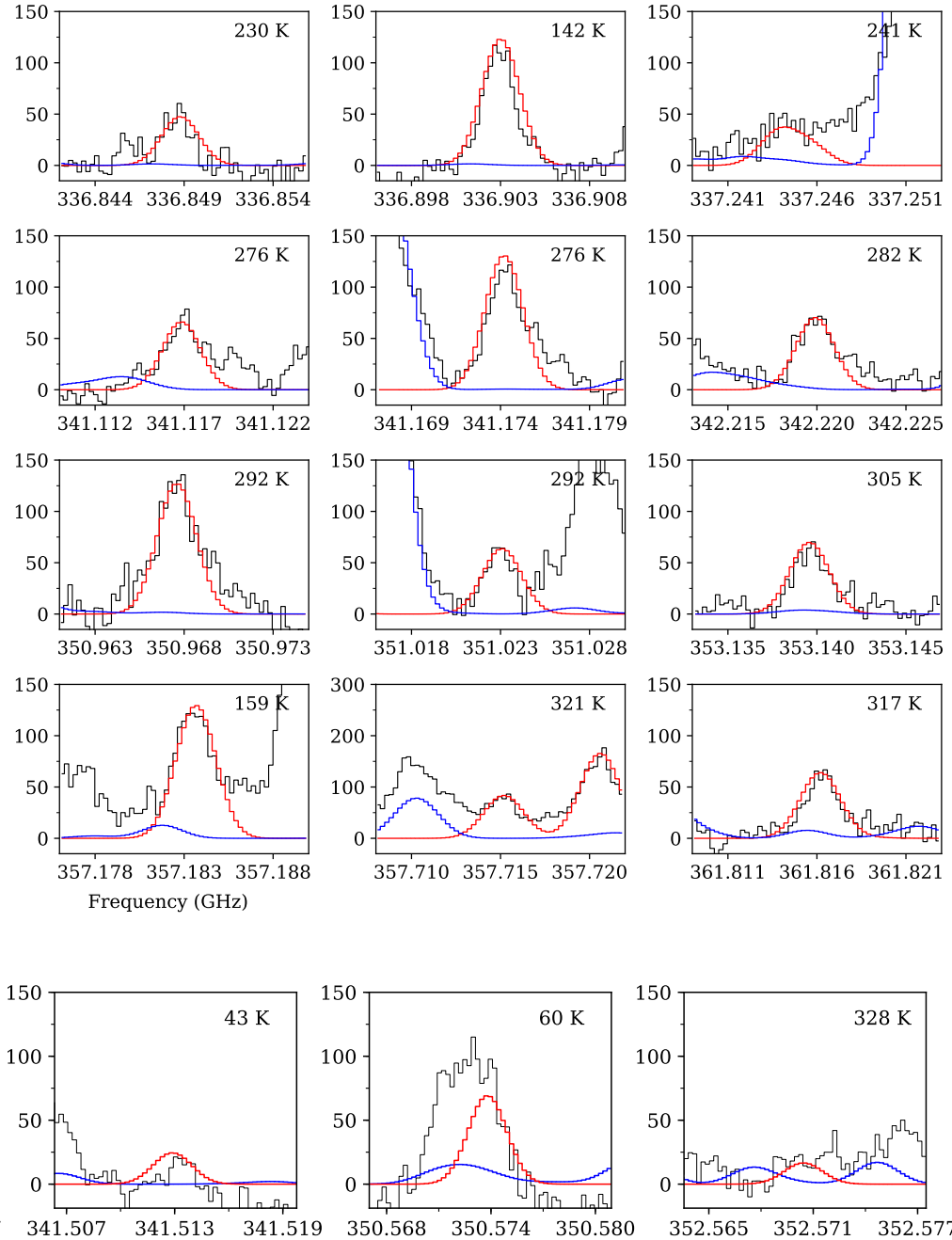

\section{$\mathrm{CH}_{2}(\mathrm{OH}) \mathrm{CHO}$}

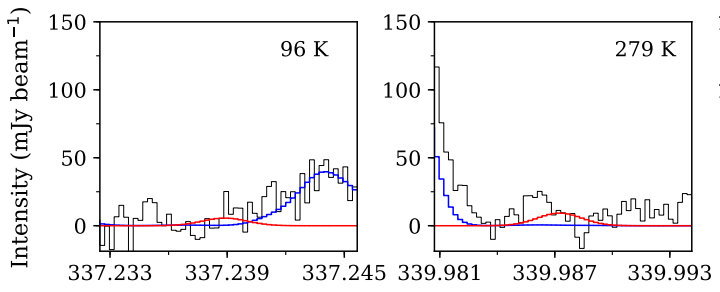

Frequency $(\mathrm{GHz})$
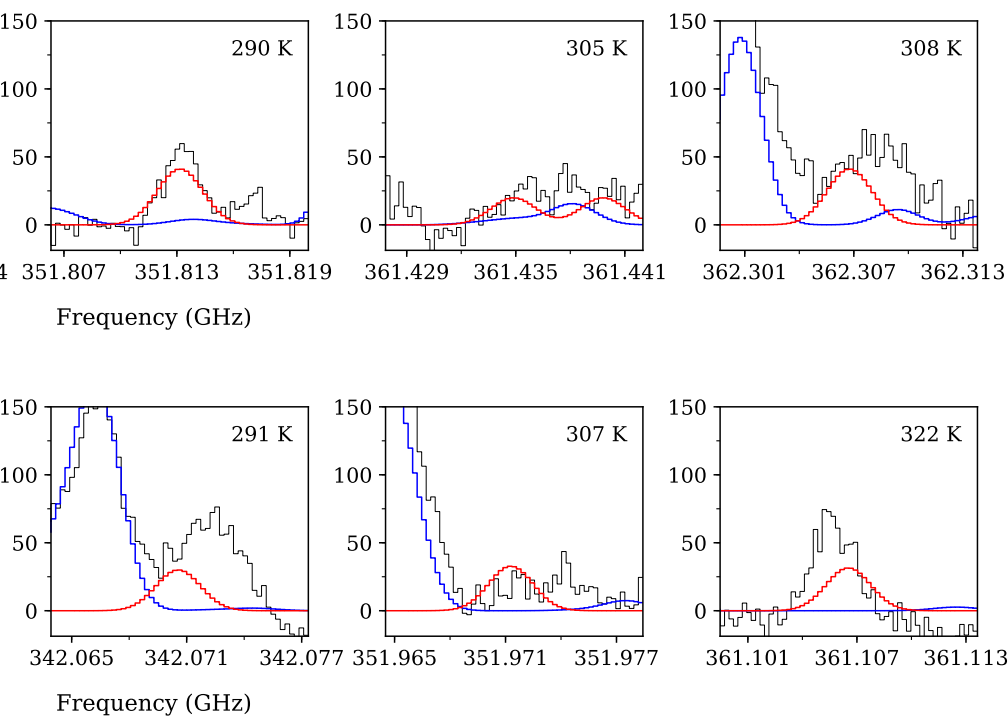

Fig. E.7. Representative selection of transitions of $\mathrm{CH}_{3} \mathrm{COCH}_{3}, \mathrm{c}_{-} \mathrm{H}_{2} \mathrm{COCH}_{2}, \mathrm{CH}_{3} \mathrm{O}^{13} \mathrm{CHO}$, and $\mathrm{CH} 2(\mathrm{OH}) \mathrm{CHO}$ towards IRAS $16293 \mathrm{~A}$. The synthetic spectra is over-plotted in red, the reference spectrum in blue, and the data in black. The upper energy level of the transition is indicated in the top-left corner. 
aGg'- $\left(\mathrm{CH}_{2} \mathrm{OH}\right)_{2}$
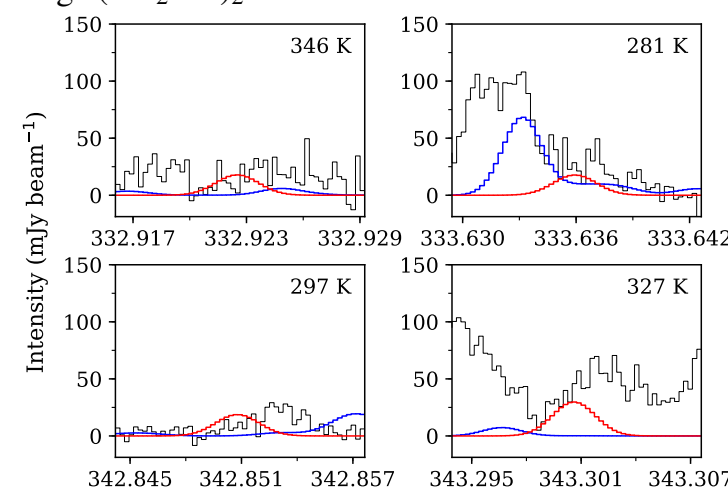

gGg'- $\left(\mathrm{CH}_{2} \mathrm{OH}\right)_{2}$
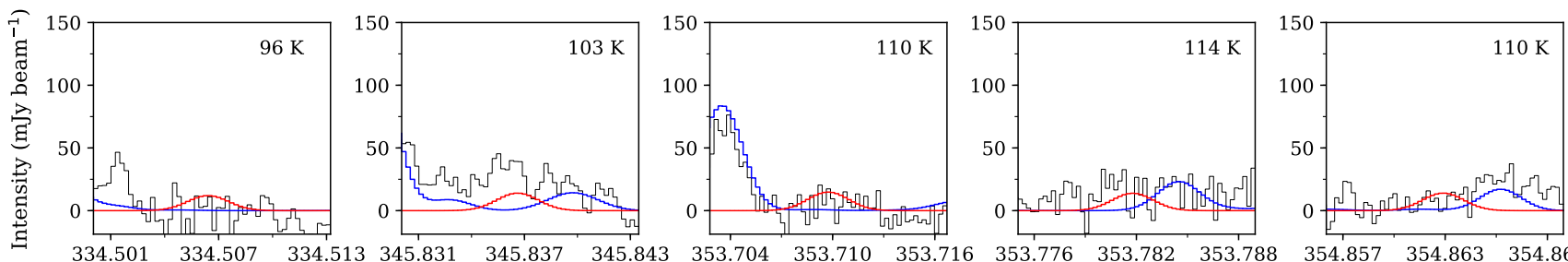
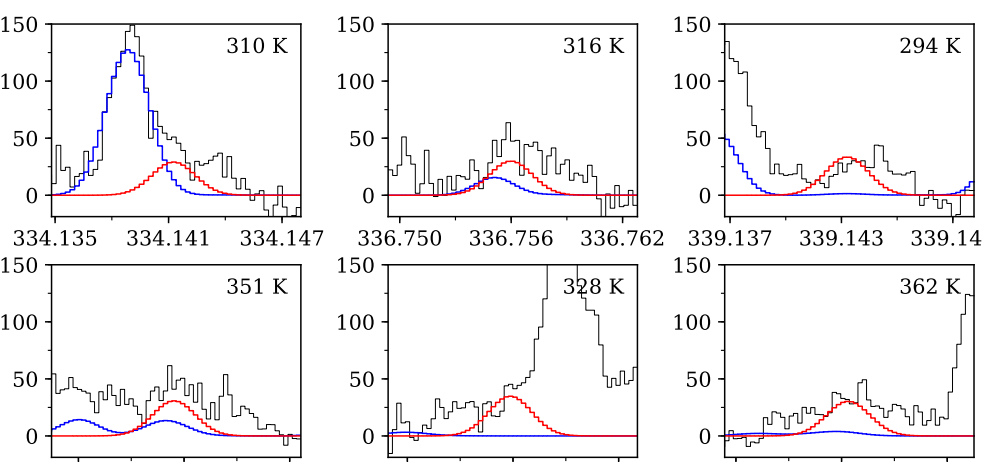

$\begin{array}{lll}357.510 & 357.516 & 357.522\end{array}$
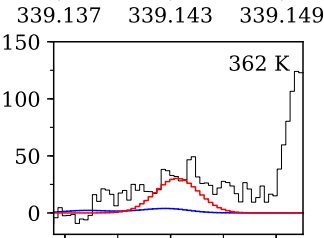

$\begin{array}{lll}361.603 & 361.609 & 361.615\end{array}$ $355.060 \quad 355.066$
Frequency $(\mathrm{GHz})$

$\begin{array}{llll}6 & 353.776 & 353.782 & 353.788\end{array}$

$\begin{array}{lll}354.857 & 354.863 & 354.869\end{array}$ Frequency $(\mathrm{GHz})$

$\mathrm{t}-\mathrm{C}_{2} \mathrm{H}_{5} \mathrm{OCH}_{3}$ (towards IRAS 16293A)
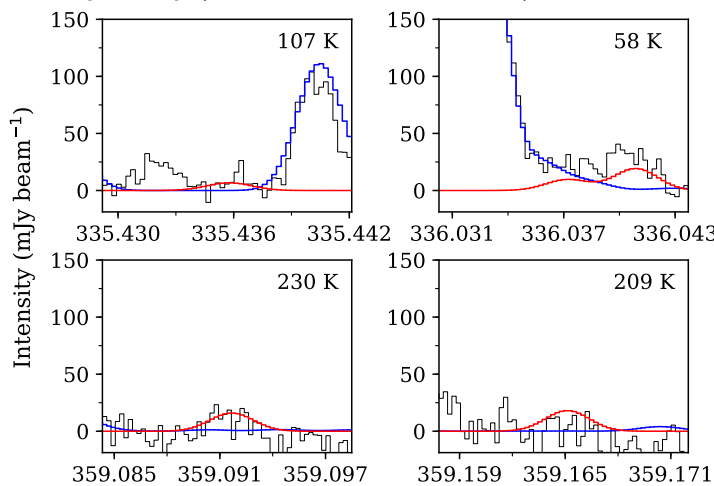
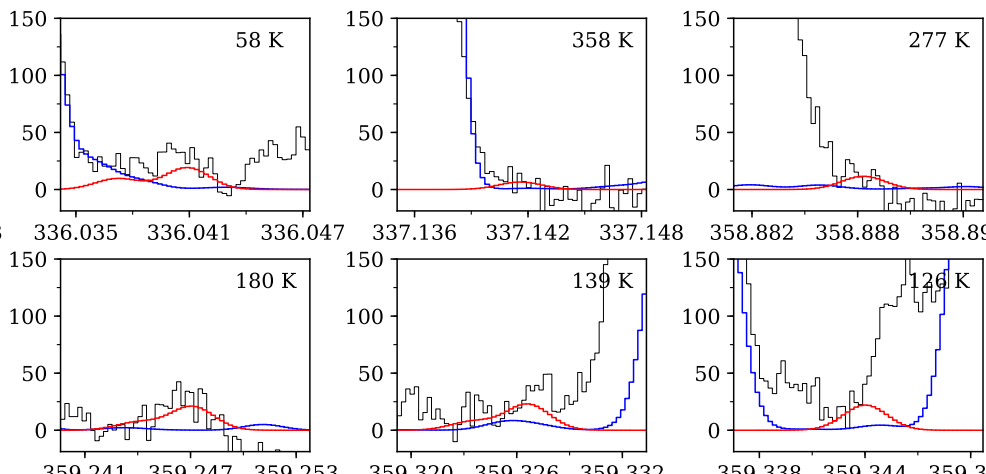

\begin{tabular}{lllllll}
\hline 359.241 & 359.247 & 359.253 & 359.320 & 359.326 & 359.332
\end{tabular} Frequency (GHz)

$\begin{array}{lll}358.882 & 358.888 & 358.894\end{array}$

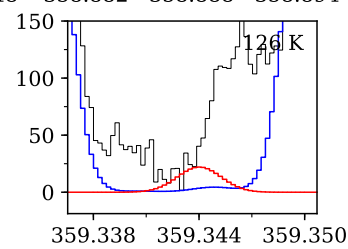

$\mathrm{t}-\mathrm{C}_{2} \mathrm{H}_{5} \mathrm{OCH}_{3}$ (towards IRAS 16293B)
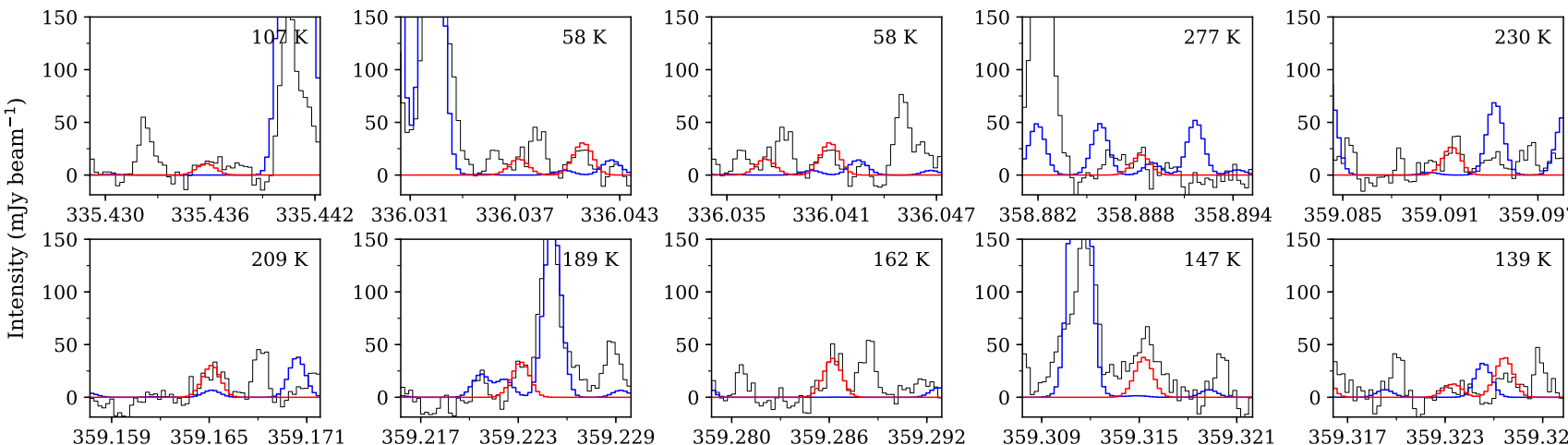

$\begin{array}{lll}359.085 & 359.091 & 359.097\end{array}$

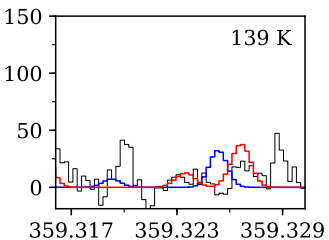

Frequency $(\mathrm{GHz})$

Fig. E.8. Representative selection of transitions of the two energetically lowest conformers of $\left(\mathrm{CH}_{2} \mathrm{OH}\right)_{2}$ towards IRAS $16293 \mathrm{~A}^{2}$ and t- $\mathrm{C}_{2} \mathrm{H}_{5} \mathrm{OCH}_{3}$ towards IRAS $16293 \mathrm{~A}$ and B. The synthetic spectra is over-plotted in red, the reference spectrum in blue, and the data in black. The upper energy level of the transition is indicated in the top-left corner. 
$\mathrm{CH}_{3} \mathrm{OCH}_{2} \mathrm{OH}$ (towards IRAS 16293A)
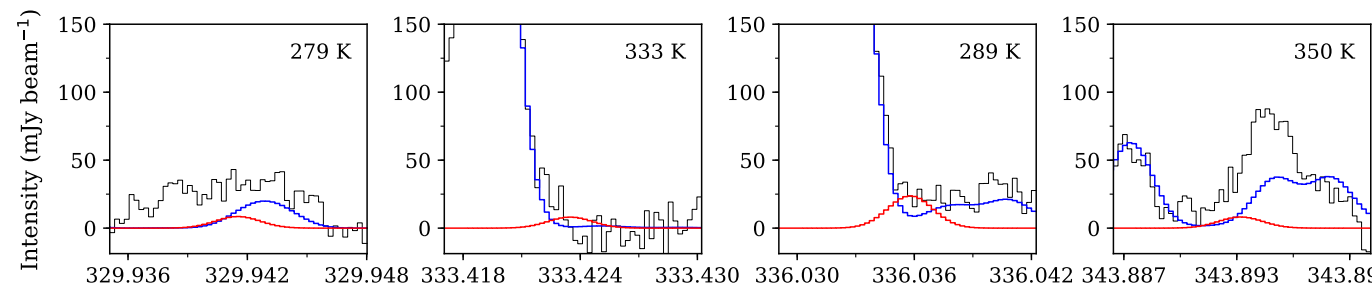

Frequency $(\mathrm{GHz})$

\section{$\mathrm{CH}_{3} \mathrm{OCH}_{2} \mathrm{OH}$ (towards IRAS 16293B)}
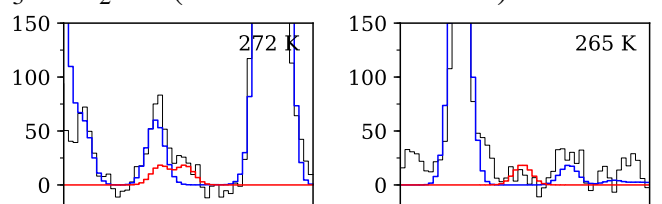

$\begin{array}{lllllll}1 & 330.267 & 330.273 & 330.279 & 332.351 & 332.357 & 332.363\end{array}$
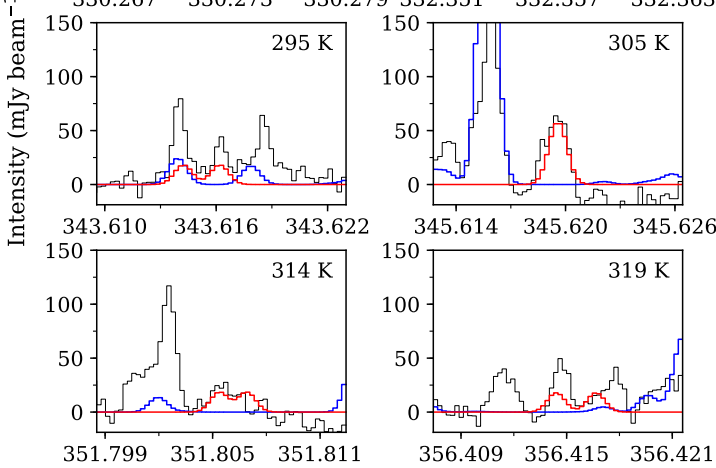

$\mathrm{NH}_{2} \mathrm{CHO}$
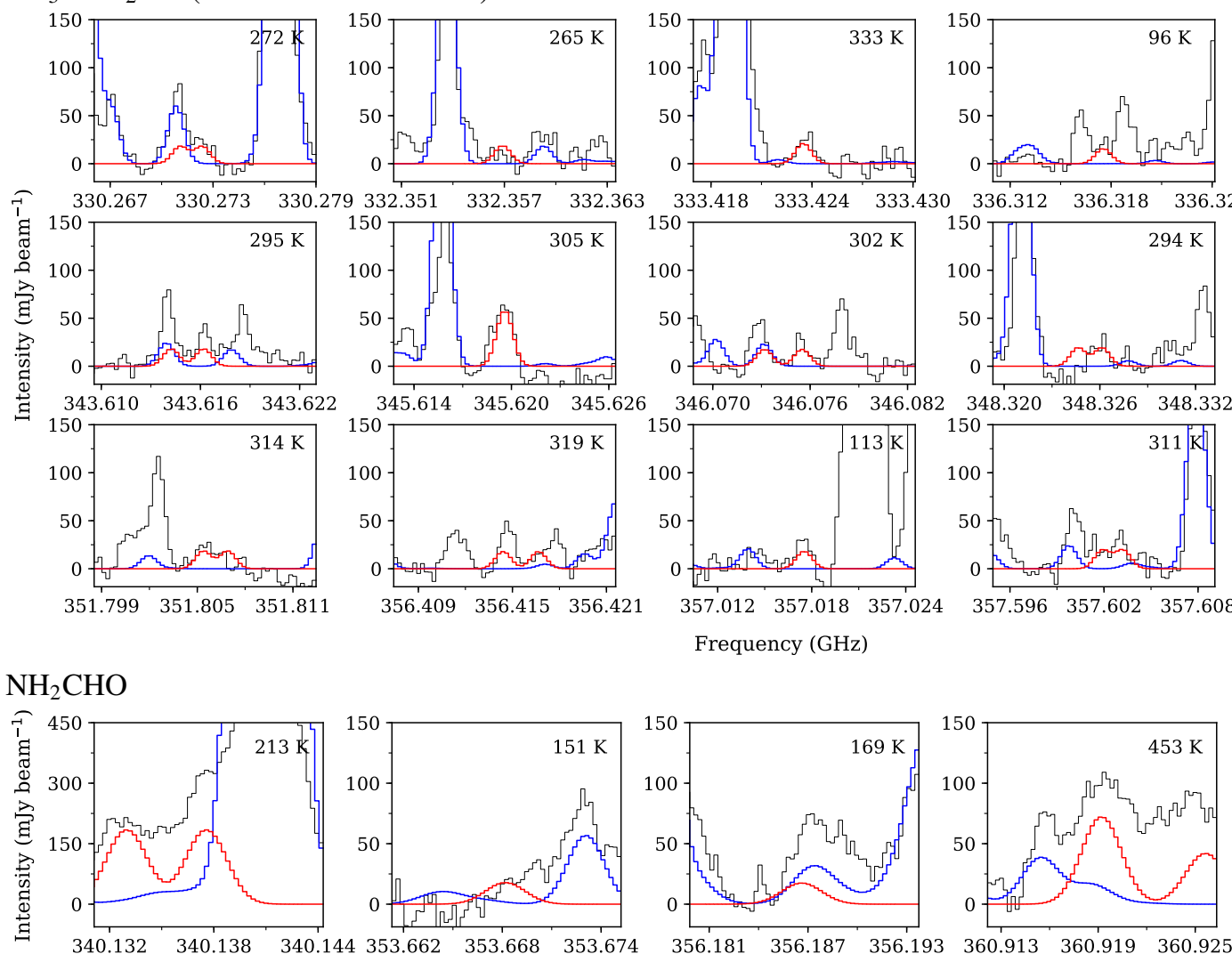

Frequency $(\mathrm{GHz})$
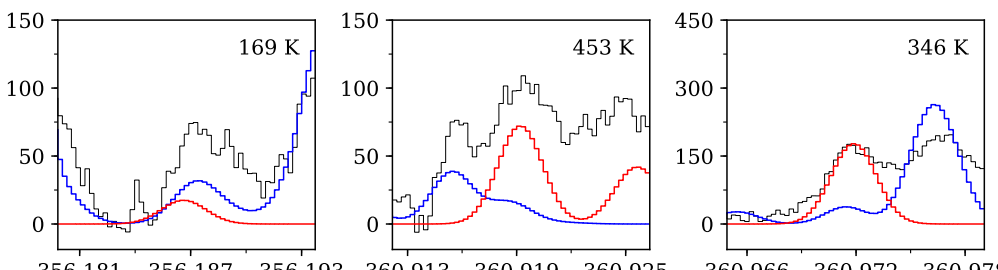

Frequency $(\mathrm{GHz})$

\section{$\mathrm{C}_{2} \mathrm{H}_{5} \mathrm{CHO}$}
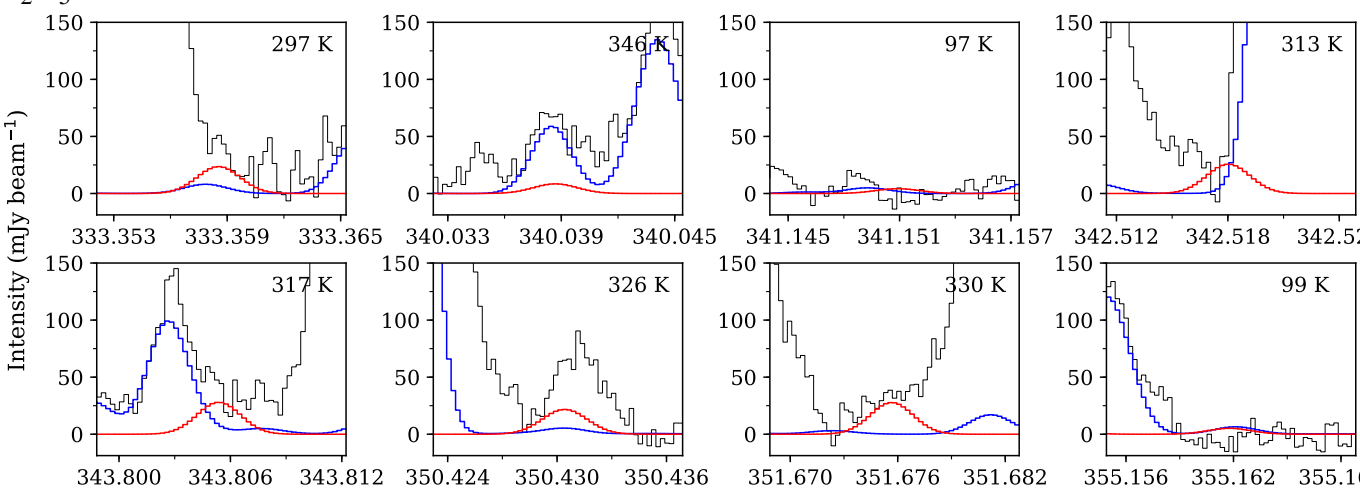

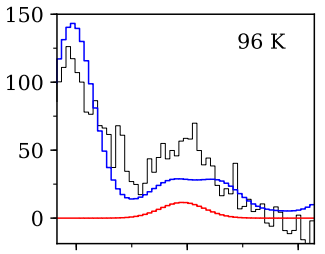

$353.186 \quad 353.192 \quad 353.198$

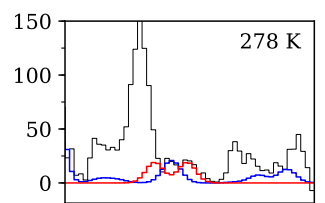

$\begin{array}{lll}338.936 & 338.942 & 338.948\end{array}$
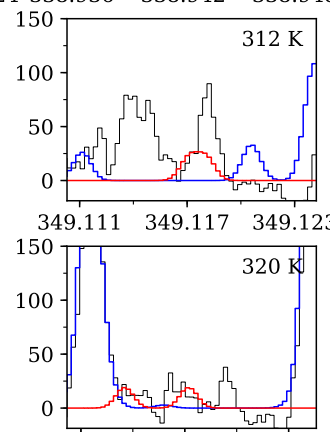

$\begin{array}{lll}362.740 & 362.746 & 362.752\end{array}$
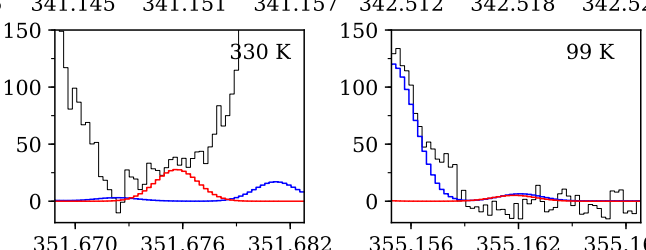

Frequency $(\mathrm{GHz})$

Fig. E.9. Representative selection of transitions of $\mathrm{CH}_{3} \mathrm{OCH}_{2} \mathrm{OH}$ towards IRAS $16293 \mathrm{~A}$ and $\mathrm{B}$ and $\mathrm{NH}_{2} \mathrm{CHO}$ and $\mathrm{C}_{2} \mathrm{H}_{5} \mathrm{CHO}$ towards IRAS 16293 A only. The synthetic spectra is over-plotted in red, the reference spectrum in blue, and the data in black. The upper energy level of the transition is indicated in the top-left corner. 
$\mathrm{CH}_{2}$ DCHO (towards IRAS 16293A)
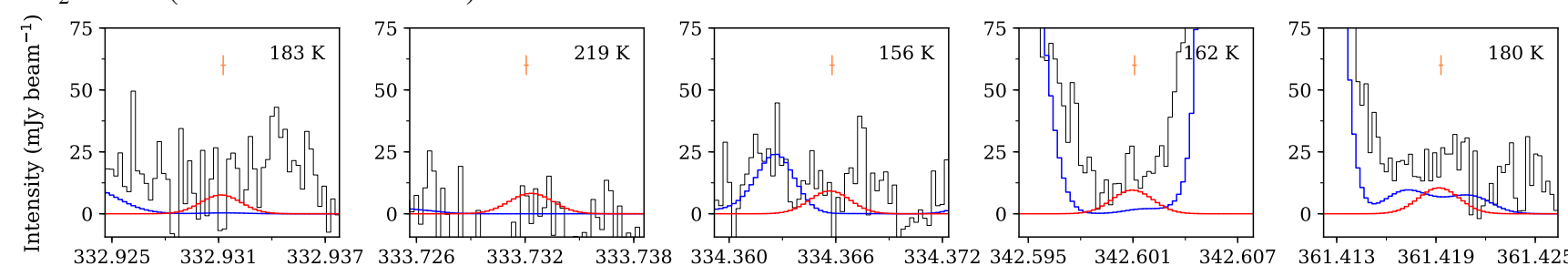

Frequency $(\mathrm{GHz})$

\section{$\mathrm{CH}_{2}$ DCHO (towards IRAS 16293B)}
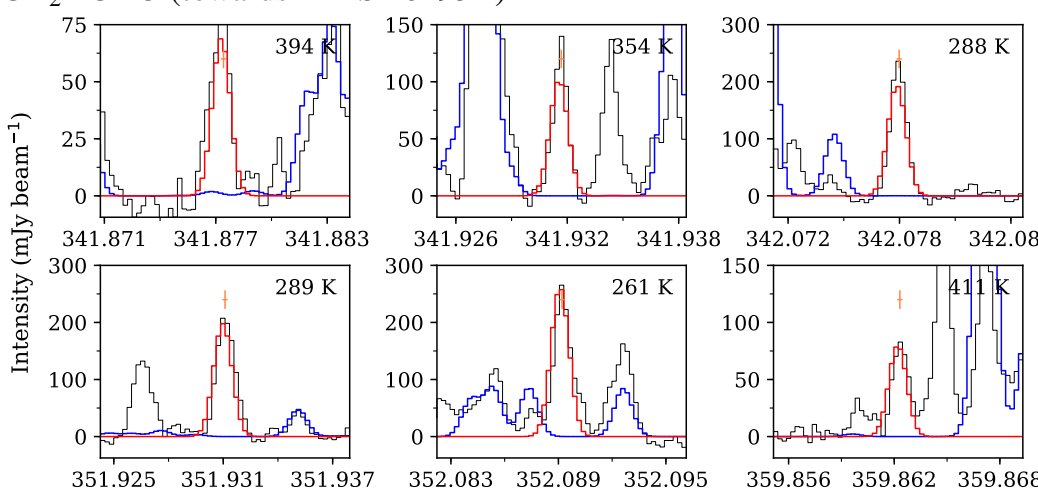

\section{$\mathrm{CH}_{3} \mathrm{CDO}$ (towards IRAS 16293A)}
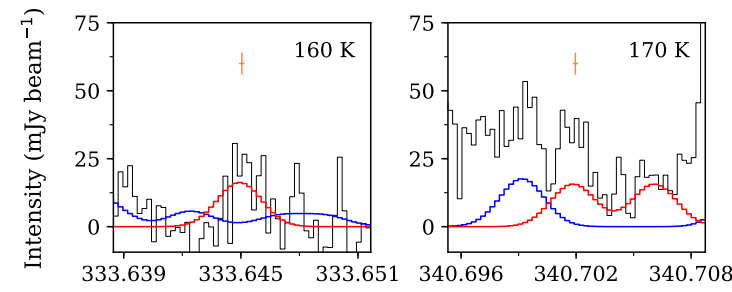

\section{$\mathrm{CH}_{3} \mathrm{CDO}$ (towards IRAS 16293B)}
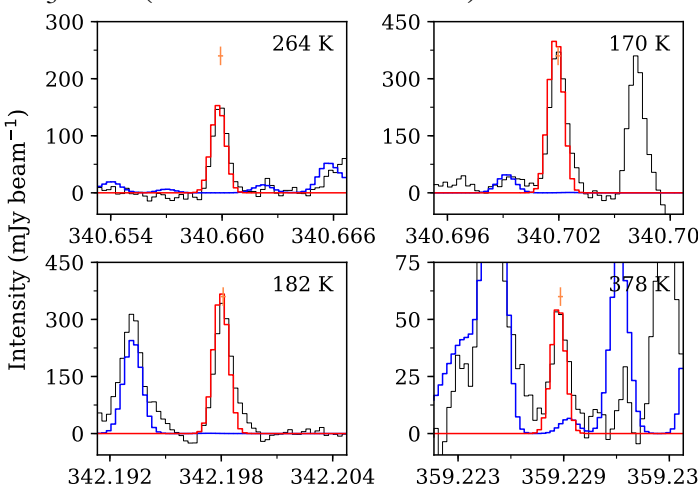

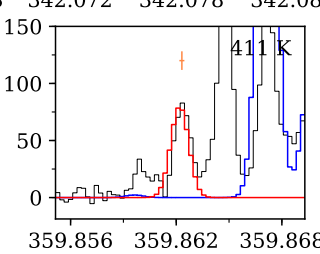

Frequency $(\mathrm{GHz})$
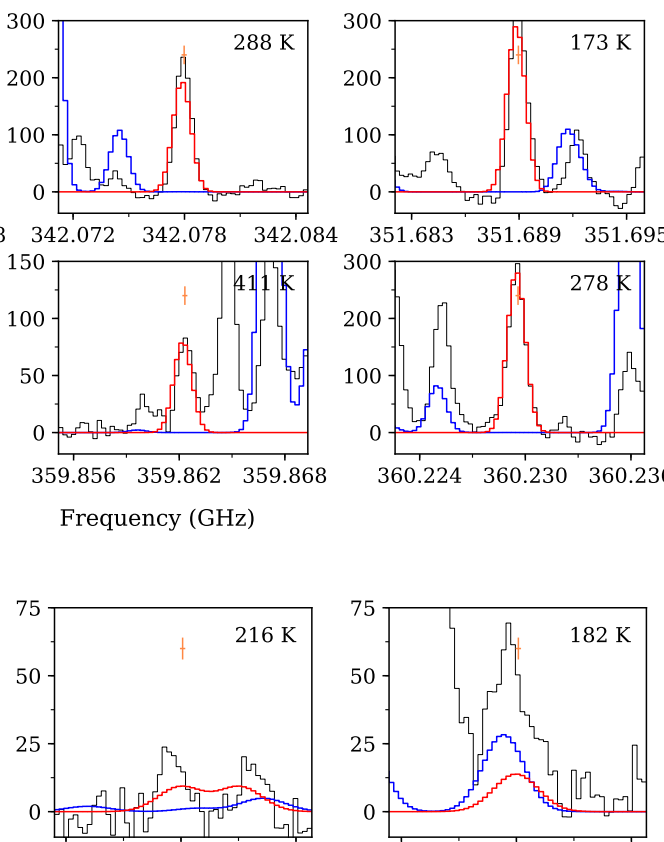

$\begin{array}{lll}360.224 & 360.230 & 360.236\end{array}$
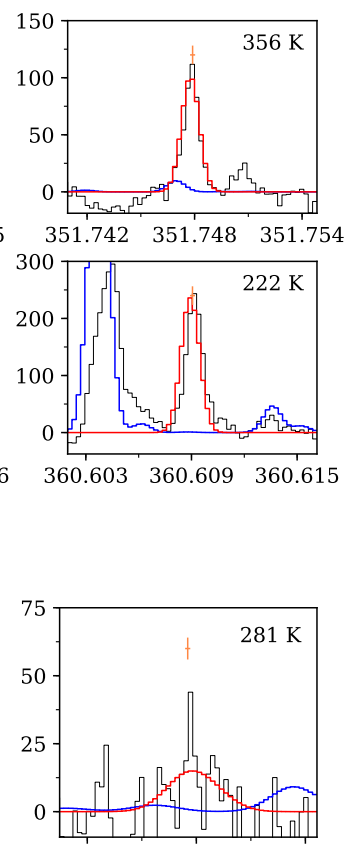

$359.601 \quad 359.607 \quad 359.613$
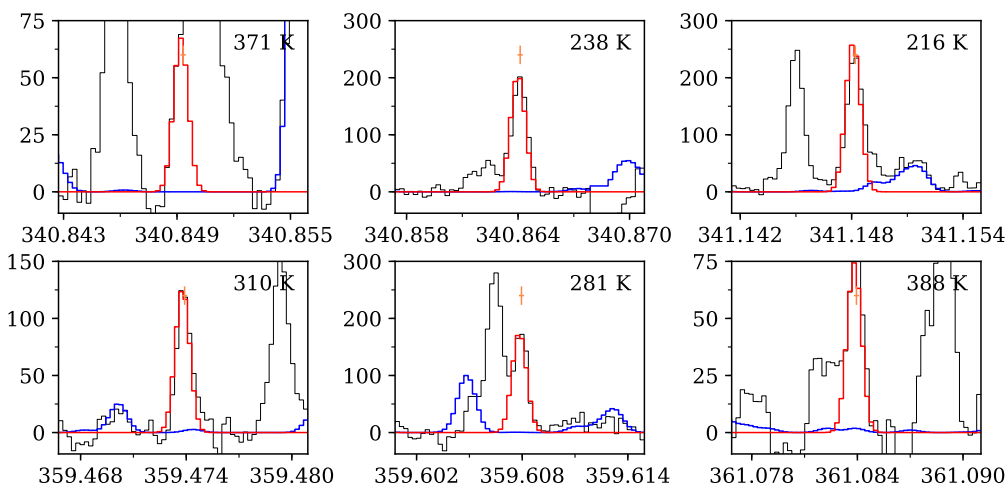

$341.142 \quad 341.148 \quad 341.154$

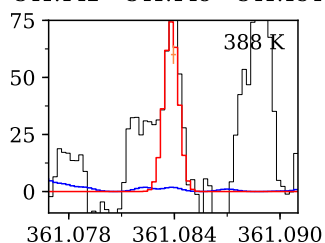

Frequency $(\mathrm{GHz})$

Fig. E.10. Representative selection of transitions of $\mathrm{CH}_{2} \mathrm{DCHO}$ and $\mathrm{CH}_{3} \mathrm{CDO}$ towards IRAS $16293 \mathrm{~A}$ and B. The synthetic spectra is over-plotted in red, the reference spectrum in blue, and the data in black. The upper energy level of the transition is indicated in the top-left corner. 